Portland State University

PDXScholar

6-9-1994

\title{
The Design, Realization and Testing of the ILU of the CCM2 Using FPGA Technology
}

David W. Foote

Portland State University

Follow this and additional works at: https://pdxscholar.library.pdx.edu/open_access_etds

Part of the Electrical and Computer Engineering Commons Let us know how access to this document benefits you.

\section{Recommended Citation}

Foote, David W., "The Design, Realization and Testing of the ILU of the CCM2 Using FPGA Technology" (1994). Dissertations and Theses. Paper 4703.

https://doi.org/10.15760/etd.6587

This Thesis is brought to you for free and open access. It has been accepted for inclusion in Dissertations and Theses by an authorized administrator of PDXScholar. Please contact us if we can make this document more accessible: pdxscholar@pdx.edu. 


\section{THESIS APPROVAL}

The abstract and thesis of David W. Foote for the Master of Science in Electrical and Computer Engineering were presented June 9, 1994, and accepted by the thesis committee and the department.

\section{COMMITTEE APPROVALS:}

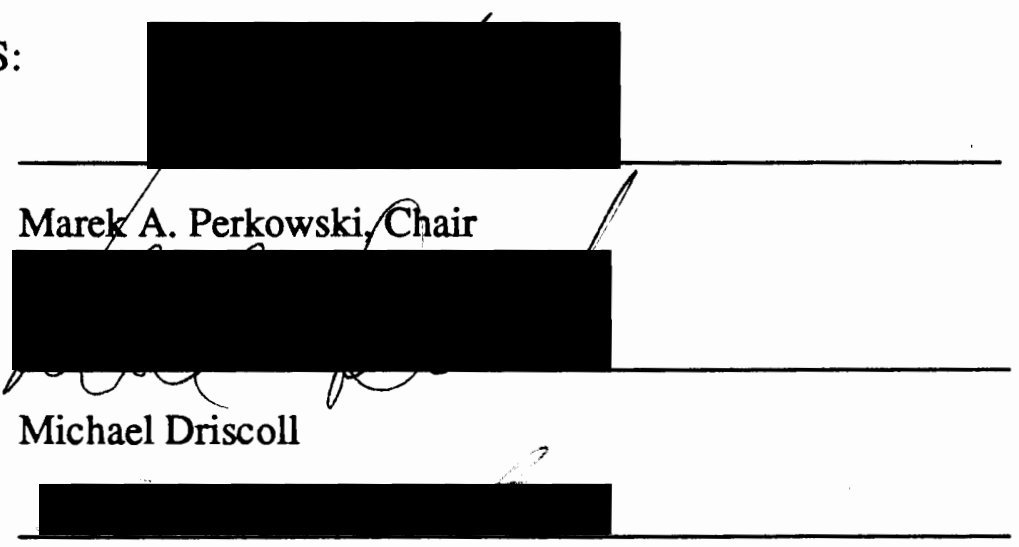

Tom Schubert

Representative of the Office of Graduate Studies

DEPARTMENT APPROVAL:

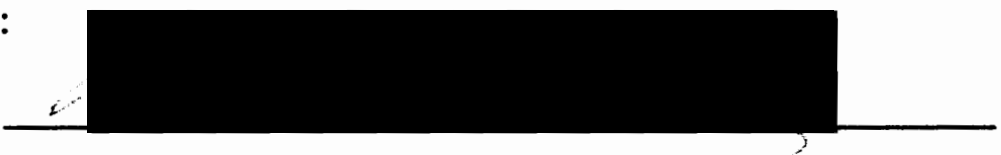

Rolf Schaumann, Chair

Department of Electrical Engineering

$* * * * * * * * * * * * * * * * * * * * * * * * * * * * * * * * * * * * * * * * * * * * * * * *$

ACCEPTED FOR PORTLAND STATE UNIVERSITY BY THE LIBRARY

by on

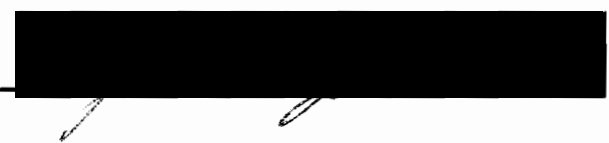




\begin{abstract}
An abstract of the thesis of David W. Foote for the Master of Science in Electrical and Computer Engineering presented June 9, 1994.
\end{abstract}

Title: The Design, Realization and Testing of the ILU of the CCM2 Using FPGA Technology.

Most existing computers today are built upon a subset of the arithmetic system which is based upon the foundation of set theory. All formal systems can be expressed in terms of arithmetic and logic on current arithmetic computers through an appropriate model, then work with the model using software manipulation. However, severe speed degradation is the price one must pay for using a software-based approach, making several high-level formal systems impractical.

To improve the speed at which computers can implement these high-level systems, one must either design special hardware, implementing specific operations much like math and image processing coprocessors, or execute operations upon multiple processors in a parallel fashion. Due to the increase in developing applications for the manipulation of logic functions, an interest in the logic machine has arisen. Many applications such as logic optimization, simulation, pattern recognition and image processing can be better implemented with a logic machine.

This thesis proposes the design, hardware realization, and testing of the iterative logic unit (ILU) of the Cube Calculus Machine II (CCM2). The CCM2 is a general- 
purpose computer with an architecture that emphasizes a data path designed to execute operations of cube calculus, a popular algebraic model used in the minimization of Boolean functions. The ILU is an iterative logic array of cells (ITs) using internal distributed control, enabling the execution of basic cube operations, while the Control Unit (CU) handles global signals from the host computer.

The ILU of the CCM2 has been realized in hardware using Xilinx Logic Cell Arrays (LCAs). FPGAs offer the logic density and versatility of gate arrays, with the off-theshelf availability and time-to-market advantages of standard user-programmable devices. These devices can be reconfigured, allowing multiple revisions and future design generations to accommodate the same device, thus saving design and production costs, an ideal solution to the resource and financial problems plaguing the University environment. 
THE DESIGN, REALIZATION AND TESTING OF THE ILU

OF THE CCM2 USING FPGA TECHNOLOGY

\author{
by \\ DAVID W. FOOTE
}

A thesis submitted in partial fulfillment of the requirements for the degree of

\author{
MASTER OF SCIENCE \\ in \\ ELECTRICAL AND COMPUTER ENGINEERING
}

Portland State University

1994 


\section{ACKNOWLEDGEMENTS}

When taking the opportunity to advance my knowledge in the field of electrical engineering, many paths were explored on the road to reaching my final destination. Many people have helped me in choosing the right paths, keeping me pointed in the right direction and reiterating the importance of the final goal. I would first like to thank my parents for all of their emotional and of course financial support throughout this time. I have learned many valuable lessons from them, but none more important than "don't ever give up on something that has not been completed to your satisfaction." I have used this lesson in many applications in my life and have yet to regret doing so. Next, a large thanks to Dr. Marek Perkowski who's creativity, encouragement and flexibility has brought me to where I am today. He has believed in me from the start, listening to my ideas and pushing me to follow up on them. I would like to thank the rest of my committee, Dr. Michael Driscoll and Dr. Tom Schubert. Dr. Driscoll has opened his door to me many times, listening, giving support and clearing the cobwebs; Dr. Schubert for agreeing to help me, not knowing me from Adam. Your generosity and insight has been greatly appreciated. Then, to Doug Hall, Mark Carlton, George LaBelle and Cathryn Scott for giving me the chance to prove myself in industry. I have learned many lessons from all of you and have gained much support as well. Finally, I would like to thank Laura Riddel and Shirley Clark for their continued support and help throughout my collegiate career here at Portland State University. 


\section{TABLE OF CONTENTS}

\section{PAGE}

ACKNOWLEDGEMENTS i

LIST OF TABLES $\mathrm{v}$

LIST OF FIGURES vi

\section{CHAPTER}

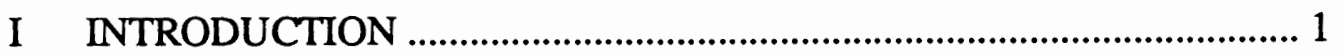

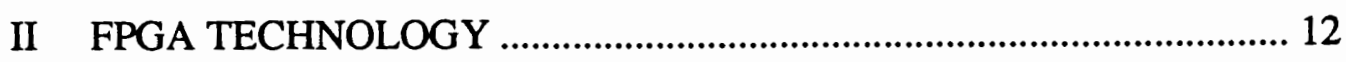

Selecting the Right Device .......................................................... 14

LCA Architecture …………...................................................... 15

Configuration Memory

I/O Block

Configurable Logic Block

Programmable Interconnect

Programming the LCA

Design Flow

Configuring the Device

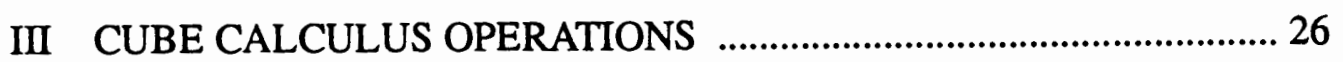

Sequential Operations .................................................................. 29

Crosslink

Non-Disjoint Sharp

Disjoint Sharp

Symmetric Consensus

Asymmetric Consensus 
Simple Combinational Operations

Intersection

Supercube

Complex Combinational

Prime

IV ITERATTVE LOGIC UNIT 45

Instruction Register 47

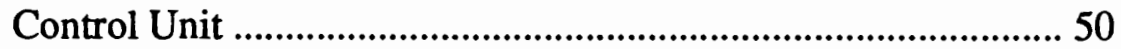

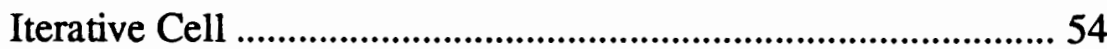

Relation

Variable Generate

State

Count

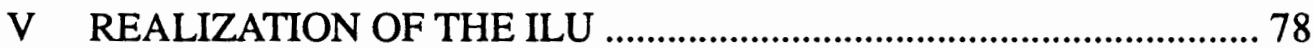

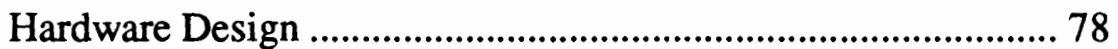

Converting the Design ................................................................... 85

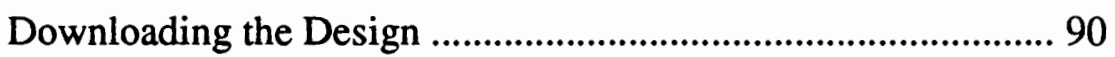

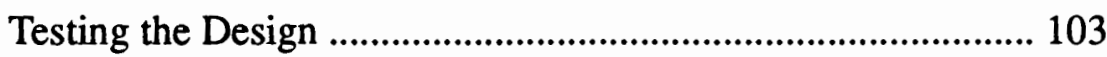

VI DESIGN EVALUATION AND COMPARISON ................................ 114

Timing Analysis ....................................................................... 114

Satisfiability

Tautology

BDD Comparison ...................................................................... 129

VII CONCLUSIONS AND FUTURE WORK ........................................ 133

Conclusions .............................................................................. 133

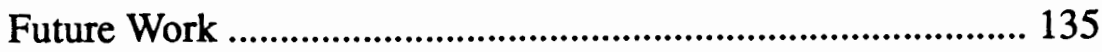

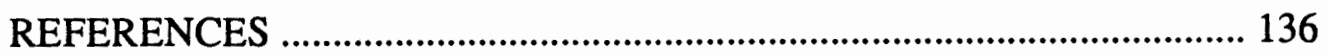


APPENDICES

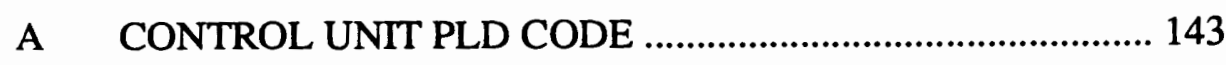

B LOGIC CELL ARRAY PLACEMENT REPORT .................... 146

C LOGIC CELL ARRAY TIMING DELAYS ............................ 151

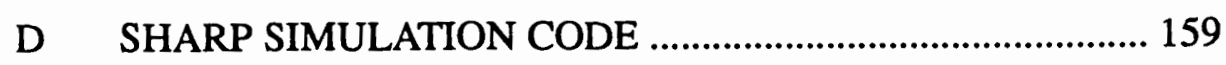

E XACT DEVELOPMENT SYSTEM TUTORIAL ..................... 163 


\section{LIST OF TABLES}

TABLE

PAGE

I Definition of sequential operation ........................................................... 49

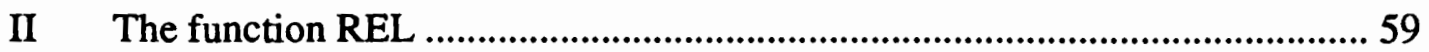

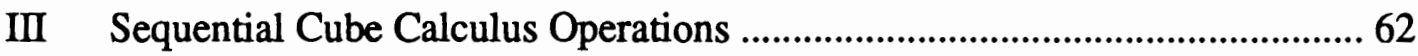

IV Sequential Cube Calculus Operations bit-wise values ..................................63

V Simple Combinational Cube Calculus Operations ........................................ 64

VI Complex Combinational Cube Calculus Operations ...................................... 64

VII Resultant cube values dependent on test values $\mathrm{T} 0$ and $\mathrm{T} 1$.........................6 65

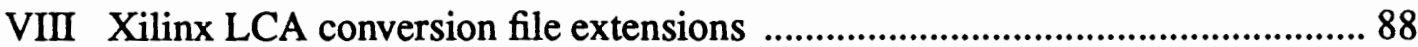

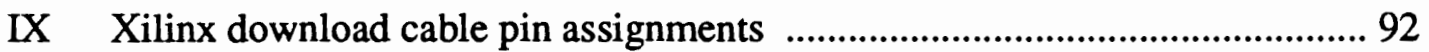

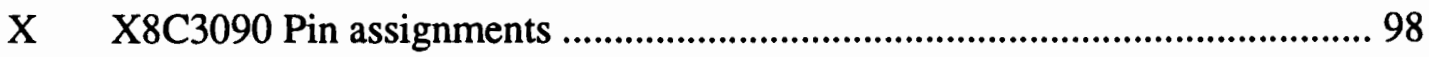

XI Differing Variable Size Sharp Comparison .................................................. 124

XII 3-Variable Sharp Comparison …............................................................ 125 


\section{LIST OF FIGURES}

FIGURE

PAGE

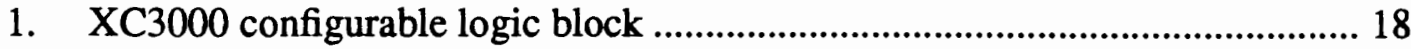

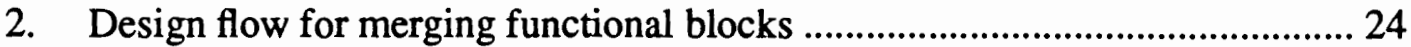

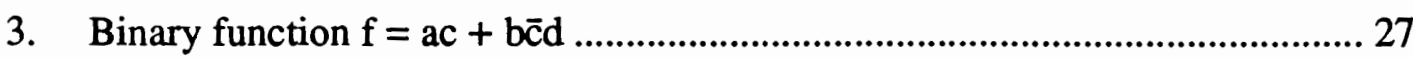

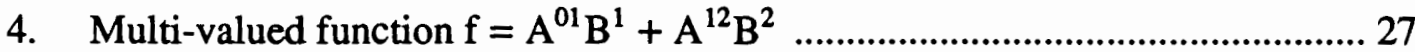

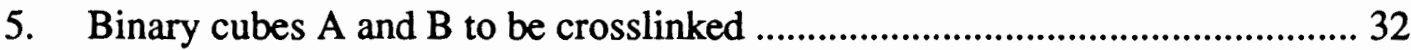

6. Four resultant cubes from the crosslink function .......................................32

7. Input cubes for the sharp operation $\mathrm{U}^{1234} \cdot \mathrm{Z}^{12} \# \mathrm{U}^{23} \cdot \mathrm{Z}^{23} \ldots \ldots \ldots \ldots \ldots \ldots \ldots \ldots \ldots . . . . . . . . . . . . . .34$

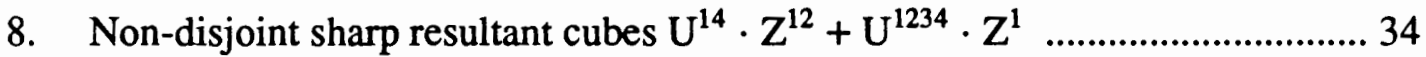

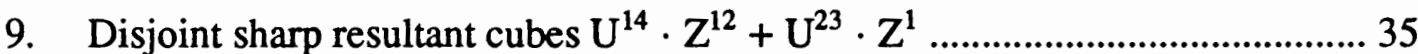

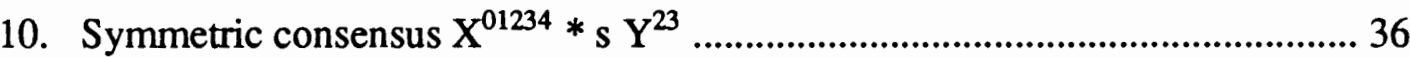

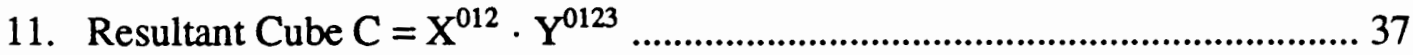

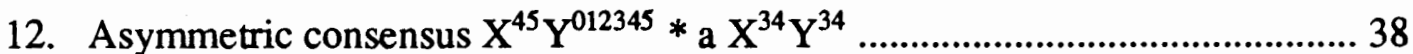

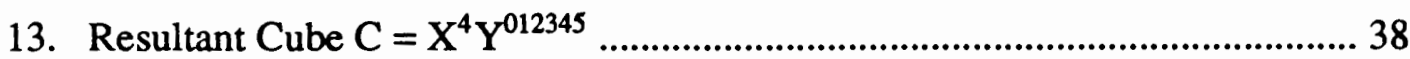

14. Input cubes $A$ and $B$ to be intersected ............................................................ 41

15. Resultant cube $C=A \cap B$

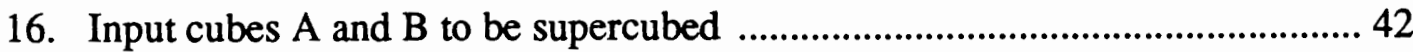

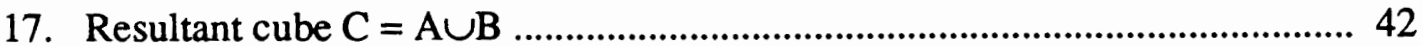




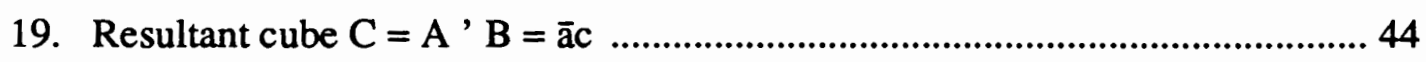

20. Cube Calculus Machine architectural model ..................................................46

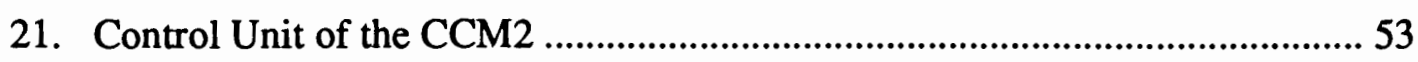

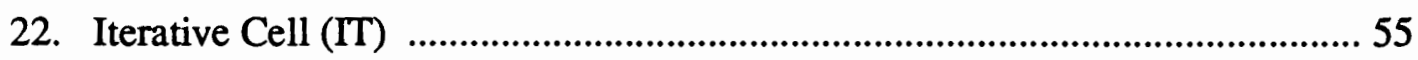

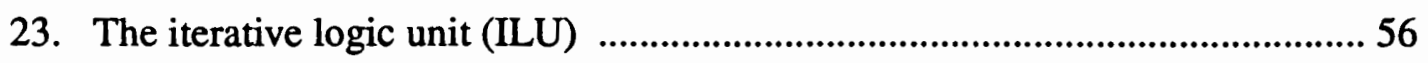

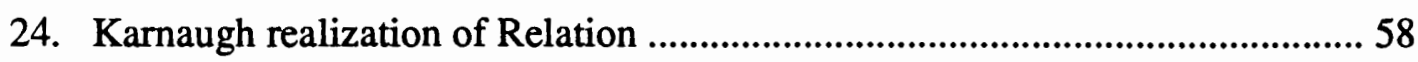

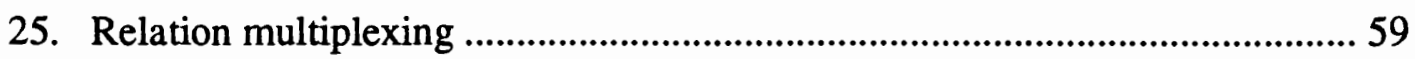

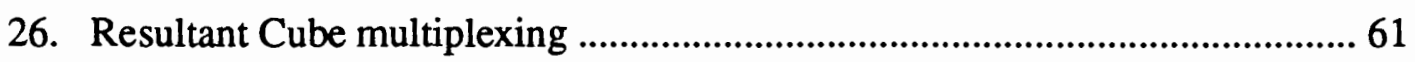

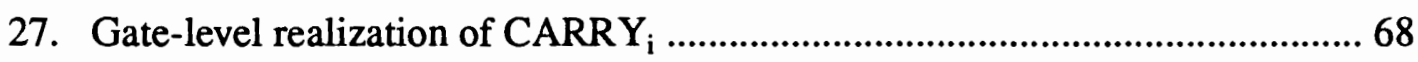

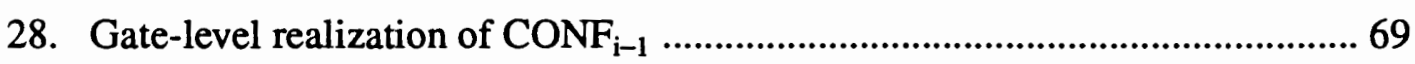

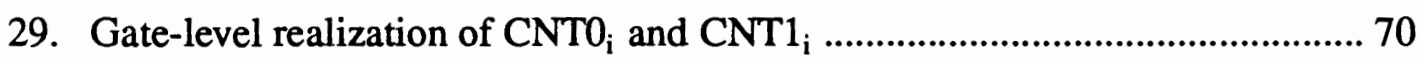

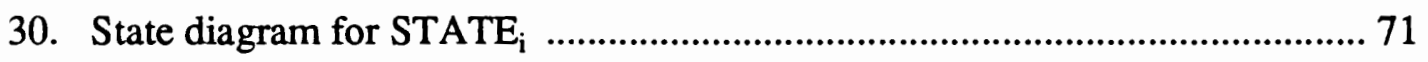

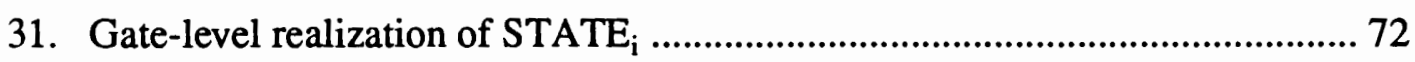

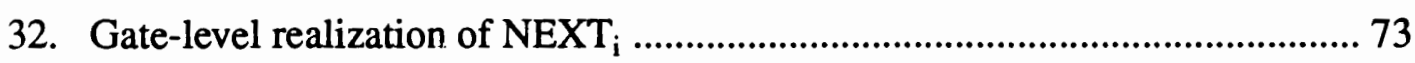

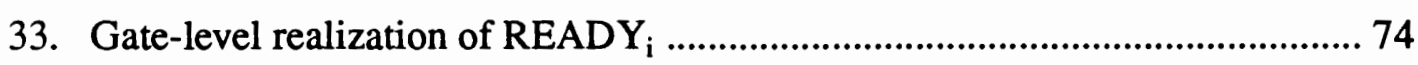

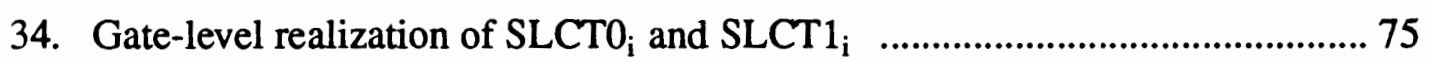

35. Gate-level realization of block COUNT ................................................... 77

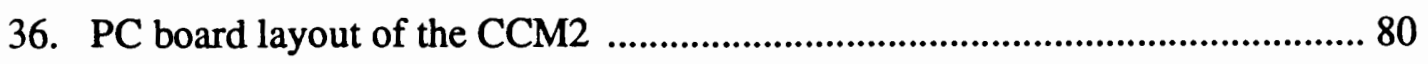

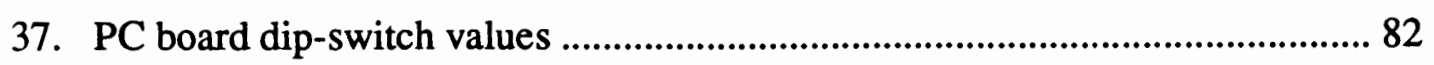

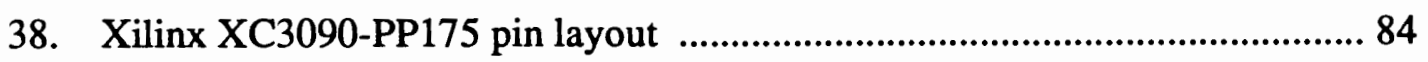

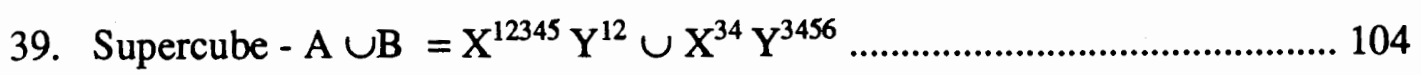

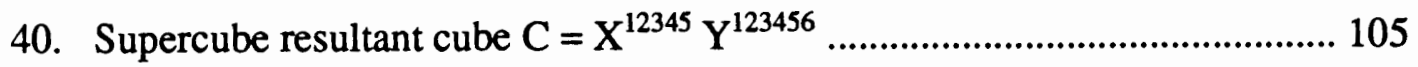




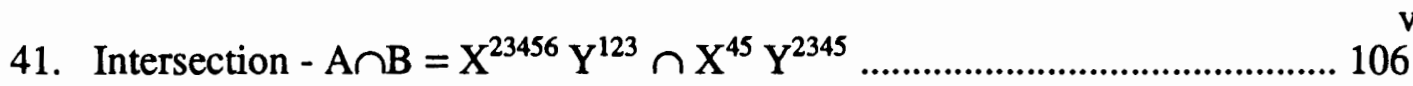

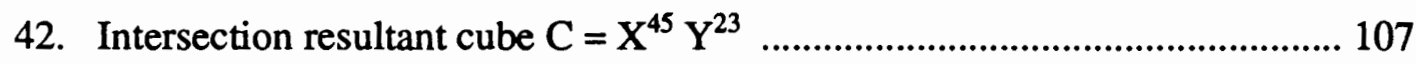

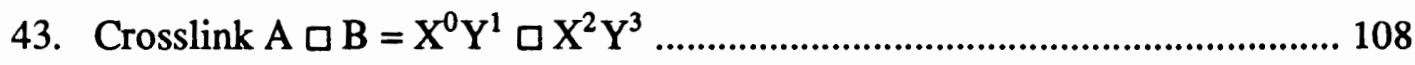

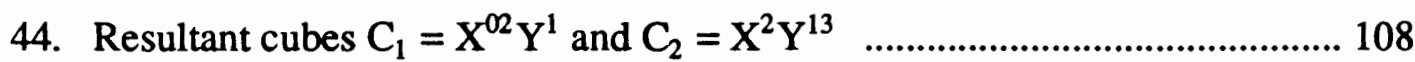

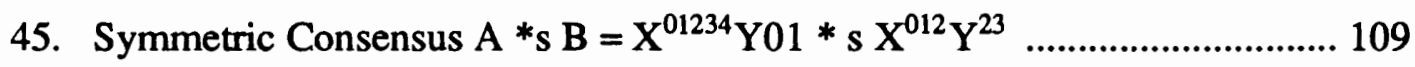

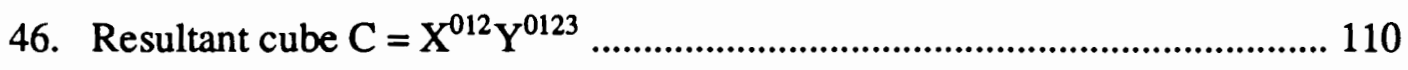

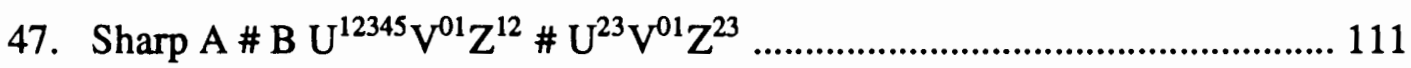

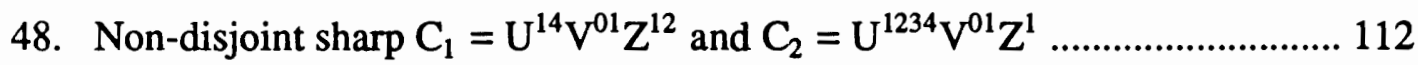

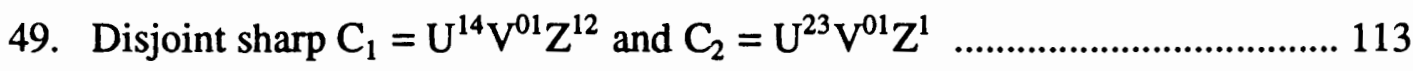

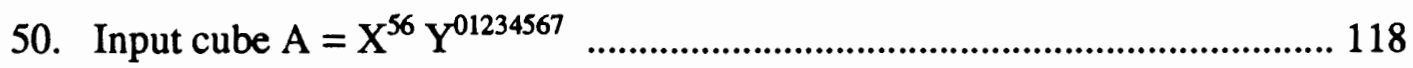

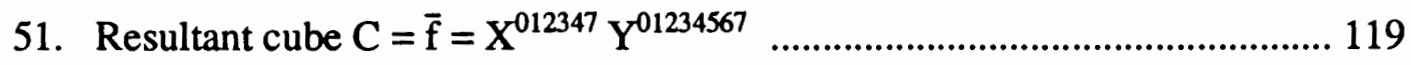

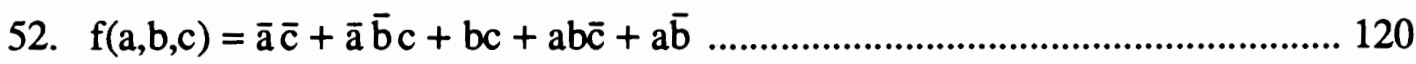

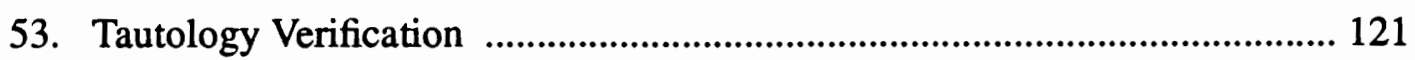

54. Multi-variable sharp comparison …........................................................... 127

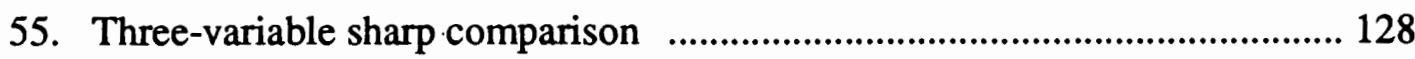

56. Function $\mathrm{F}=\mathrm{A}+\overline{\mathrm{BC}}$ in $\mathrm{BDD}$ representation. ....................................... 129 


\section{CHAPTER I}

\section{INTRODUCTION}

A formal system is a set of symbols with an associated set of operations that may be performed upon those symbols. Most existing computers today are built upon a subset of the arithmetic system which in turn is based upon the foundation of the set theory. All formal systems can be expressed in terms of arithmetic and logic operations implemented on current arithmetic computers through an appropriate model and software manipulation upon that model. Severe speed degradation however is the price one must pay for using a software-based approach, making several high-level formal systems impractical. To improve the speed at which computers can implement these high-level systems, one must either design special hardware, implementing specific operations much like math and image processing co-processors, or execute operations upon multiple processors in a parallel fashion.

This thesis proposes the design, hardware realization, and testing of the iterative logic unit (ILU) of the Cube Calculus Machine II (CCM2) [13][30]. The first version of the CCM was introduced by Luis S. Kida and Dr. Marek Perkowski as a general purpose logic computer with a data path designed specifically to execute operations of Cube $\mathrm{Cal}-$ culus (CC), an algebraic model used in the minimization of Boolean functions [12][13][17][30][42][44][51][52][53]. The machine uses a positional cube representation allowing the expansion to multiple-valued $(\mathrm{mv})$ input algebra. This $\mathrm{mv}$ algebra finds many recent applications in logic synthesis, for instance: PLA design and state assignment of finite state machines (FSMs). The processing unit (ILU) of the CCM2 is a 
network of iterative cells (ITs), each containing an asynchronous FSM. Each IT processes two bits, either as a binary variable, or a portion of a multi-valued variable. Each IT passes iterative signals from left to right and right to left, with each individual FSM storing the position of the IT in relation to the active literal which is being processed. An example of a similar circuit would be that of a sequential parity checker. The machine may be daisy-chained to extend the ILU to operate on larger input cubes.

The CCM2 processor can execute a very wide range of operations, which include all cube calculus operations such as the sharp of two arrays of cubes and the complementation of a Boolean function. It allows the realization of any operation of multiple-valued "set-theoretical" input, binary output logic, as well as any operation of the "truly multiple valued" (where both inputs and outputs are multiple valued) 3-valued and 4-valued logics. It therefore efficiently executes operations such as the satisfiability problem, the generalized satisfiability problem and the tautology problem [37]. This makes the CCM2 an excellent hardware accelerator for applications such as logic synthesis and logic minimization, but also for combinational operations in image processing and other areas $[2][10][19][28][47][50]$.

Since the CCM2 was specifically designed for cube calculus operations, these operations can be executed in just a few clock pulses and require only one CCM2 instruction. The CCM2 does not generate empty resultant cubes, so the output of resultant cubes is completely regular. The time needed to generate the cubes solely depends on the number of resultant cubes that are not empty.

A considerable part of the control of the CCM2 is implemented in its data path, which is the ILU. Once the ILU has received a CC operation, the only control that resides in the control unit $(\mathrm{CU})$ of the $\mathrm{CCM} 2$ is providing the clock signals for the generation of the resultant cubes. Thus, no external control from the host computer is 
necessary. The CCM2 processor has been optimized to efficiently solve any problem that can be represented as a multi-valued input CC operation, it allows the generation of multiple output cubes of a CC operation in just one clock pulse per resultant cube. No empty resultant cubes are generated, meaning that the output of resultant cubes does not have any irregularities. Relations and operators can be arbitrary Boolean functions of input variables.

The CCM2 is a processing element designed to be implemented as a co-processor to accelerate cube calculus operations executed from a host processor. The architecture is intended to be programmed into Field Programmable Gate Arrays (FPGAs), which can be mounted on an additional board for a host computer. A host processor is required purely for control and initialization of the operations. Thus, the CCM2 processor will function as a hardware accelerator board for the host, executing operations that would normally be implemented as subroutines in the program of the host computer.

In conventional computer architectures, the control is usually located in the program that is stored in RAM. This results in a considerable control overhead, since the instructions have to be fetched from RAM memory. If an algorithm contains loops, the same instructions will be read many times. This makes the memory interface the bottleneck of these architectures, especially when the memory bus is not as fast as the internal processor bus. In the CCM2 architecture, most of the control is implemented in the data path. Once an instruction is loaded into the CCM2, the host computer only needs to write the data cubes to the CCM2 and read the resultant cubes back from the CCM2. The host processor can process the resultant values from the CCM2 while loading them from the CCM2, as the CCM2 awaits the next clock pulse to send another cube.

Conventional general purpose architectures can be used for most applications only at the cost of growing control overhead, since the ALU has only a limited set of arithmetic 
instructions. The FPGA implementation of the CCM2 allows a customized ILU instruction set, which can easily be optimized for certain applications. The SRAM based FPGAs can be reconfigured while the host computer, or even the host program that uses the $\mathrm{CCM} 2$, is in full operation.

In many commonly used computer architectures, there is very little parallel processing, even in modern RISC or Pentium processors. Parallel processing has also proven to be very hard material for compilers. In the CCM2 architecture parts of an existing program can be replaced by a single CCM2 instruction. The gain in processing speed is based upon the size and number of input variables that the ILU receives. Each IT will process 2-bits of the input variable regardless of being binary or multi-valued. This CCM2 instruction is then executed in hardware that was specifically designed for this particular instruction, allowing parallelism in the CCM2. Another limiting factor in conventional computer architectures is the bandwidth of the ALU. As a result of the FPGA implementation, the CCM2 suffers from this problem to a much lesser extent. The number of ITs in the ILU can easily be increased to obtain a higher ILU bandwidth. The only limiting factor is the capacity and speed of the FPGAs in the hardware that is used. Furthermore, the CCM2 architecture allows massive parallel architectures to be built from a large number of CCM2 processors, which are controlled by other CCM2s and, ultimately, by the host computer. Thus, true parallel processing can be realized. The mapping of these architectures onto the FPGAs requires considerable time, but once they are compiled, the host computer can instantly load new architectures into the FPGA board.

The question arises whether the speedup of certain applications justifies the purchase of a costly hardware accelerator board. However, the essence of the CCM2 is not the FPGA board, but the architecture that is programmed into the FPGAs. The CCM2 architecture can be programmed on existing FPGA-architecture hardware boards, or a 
'specific' CCM2 hardware board may be used for other applications [9][54]. The existing FPGA boards would then have the potential of targeting additional applications, allowing continued research to be done in other areas of study, thus greatly reducing the cost of the CCM2 project and other projects within the University environment.

There are several reasons why the CCM2 can greatly speed up many applications. The main reason is the fact that the Arithmetic Logic Unit (ALU) in conventional computer architectures can only compute arithmetic and simple logic functions such as AND, $\mathrm{OR}$, addition and shifting. With this set of basic functions, it is possible to perform many, more complicated, operations and thus realize a general purpose computer. For many applications, however, this method results in slow and highly inefficient operation. For instance, to calculate the consensus of two cubes, the ALU must execute a long series of shifts and ANDs. Also, some of the resultant cubes are empty and must be removed. This means that the generation of resultant cubes is irregular and inefficient.

In recent years there have been two major application areas for FPGA-based add-on boards for commonly used computers. The first of these two is rapid prototyping. During the development of new hardware, the new design is usually simulated before an actual prototyping is made. This simulation is usually very time-consuming and not completely free of errors. The construction of a prototype is also slow, expensive and errorprone. The design process can be greatly sped up by mapping the design, or parts of it, onto FPGAs on a computer add-on board. The design can then be tested as a part of the hardware it is meant, but can still be easily changed. This way most errors in the design can quickly be detected before the prototype is made. An example of such a rapid prototyping board is the 'Anyboard', an on-going project concerning reconfigurable systems developed at the University of North Carolina [54].

Another development area is the concept of an FPGA-based computer architecture. 
An example of such an architecture is the 'Xputer' which was designed by Prof. Hartenstein's group in Germany [21][22][23]. Unlike the CCM2 concept, the Xputer architecture includes the actual FPGA hardware. Almost all the logic on the Xputer hardware, including a reconfigurable ALU, is programmable. Furthermore, the Xputer implements almost all the control in the data path, using data sequencing. These two main characteristics allow very efficient architectures to be realized on the Xputer hardware. Due to the use of data sequencing, the Xputer is especially fitted for applications that involve data with some sort of regularity. These applications include systolic algorithms, image processing, physical design automation and DSP. After being tested, the Xputer architectures can be transferred to mask-programmed ASICs, which are faster than FPGA's, for mass production. The CCM2 architecture can also be programmed onto the Xputer hardware.

The fact that the CCM2 architecture can be programmed onto the Anyboard and the Xputer is not the only reason they were mentioned. Both use repeated compiling of new designs onto multiple FPGAs. This requires extensive and time-consuming logic minimization and mapping algorithms. It just happens that logic synthesis is one of the key application areas were the CCM2 processor can make a great difference in execution speed. Since the SRAM-based FPGAs can be reprogrammed while the host computer is in full operation, it is quite easy to load the FPGA board with the CCM2 processor and use the CCM2 to speed up the calculation of the new design of the board. When that design is finished, it is simply loaded into the FPGAs and immediately ready for testing.

This illustrates the advantages of an FPGA-based add-on board. One single FPGA board can be used to implement several different add-on boards and speed up many different nonconcurrent tasks. This not only reduces the costs of hardware, but also the power consumption, the number of slots that are needed, and, last but not least, even the 
size of the computer case.

The latest wave of FPGAs and Complex Programmable Logic Devices (CPLDs) give designers the capabilities of gate array devices with the design benefits found only in programmable array logic (PAL) devices. The following pages give a brief overview of the CPLD and FPGA technologies as well as insight into the major players of the programmable logic device technology advancement.

CPLDs are variations of $22 \mathrm{~V} 10$ PALs, lending themselves to old fashioned $5400 / 7400$ TTL-style, schematic capture based design. CPLDs rely on sets of macrocells (22V10 act-alikes linked with chip routing resources). The CPLD logic cores are a mix of combinatorial logic (memory look-up tables, multiplexors, or AND/OR arrays) and one or more register elements. Typical anomalies experienced when using CPLDs are the under-use of the registers and under-utilization of the core blocks.

Altera, one of the pioneers of CPLD technology, provides a windowed development environment that handles both Verilog and Altera HDL design input to the CPLDs. Cypress however is determined to become a major player in high-end programmable logic with its latest proprietary CPLD. It combines flash memory reprogrammability with a high-routability, fixed-speed interconnect linking up to 256 macrocells [55]. The devices integrate PAL-like macrocells into logic blocks, with 16 macrocells per block. The downfall of CPLDs lies in the compromise one must make to fit large numbers of macrocells.

Field Programmable Gate Arrays (FPGAs) are built around proprietary core-logic blocks that are typically a mixture of multiple gates/multiplexors and registered elements supplemented with proprietary routing resources. These devices have yet to reach ASIC efficiencies, lagging about an order of magnitude in density and speed behind due to their field programmability, large logic blocks and routing restrictions. Mainstream algorithms 
and techniques were developed for ASIC gate arrays and standard cells with their underlying gate elements [57]. To synthesize FPGAs, the algorithms must be tailored for each architecture core and routing structure.

The major CAE vendors such as Mentor Graphics, Cadence, Synopsys and Viewlogic currently supply FPGA logic-synthesis tools that provide compatibility with existing ASIC development environments, allowing designers to extend these environments to FPGAs for design and prototyping purposes. The growth of these companies has sparked a revolution from companies such as Data I/O, NeoCAD and Exemplar, providing PLD and FPGA specific tool sets promising to outperform the device's proprietary tools. Granting that the biggest opportunities lie in the CMOS ASIC market, designers will want to use the same tools to design FPGAs that they will use for gate array development.

The Xilinx SRAM-based family of Logic Cell Arrays (LCAs) holds the major share of the production margin of the FPGA industry. A recent agreement with Synopsys has added the mutual development of Hardware Description Language (HDL) synthesis tools and macro libraries for existing Xilinx devices. Xilinx's closest competitor in the FPGA marketplace is Actel, which has a device structured much like a conventional gate-array, comprising a matrix of logic modules with the rows separated by wiring channels [11]. For large combinational circuits such as the CCM2, the Actel devices with flexible, finegrain logic blocks might give higher device utilization. AT\&T's Orca FPGAs, which are extensions of the Xilinx technology, target data-path applications. AT\&T is currently working on its own advanced module generator, having extensions for RTL blocks and data flow. Atmel has also introduced the AT6000 family of FPGAs that may be dynamically reconfigured on the fly while the logic is running [56]. The technology builds upon a matrix of several small core-logic SRAM cells that can be dynamically loaded during circuit operation, much like how a computer can load a new application or thread into 
memory for execution while other processes are occurring.

Other competitors include QuickLogic and Cypress. Neither of these devices are RAM based FPGAs, however, they are highly routable parts, easing the logic-synthesis place and route. These devices also have their own proprietary core-logic blocks. The proprietary cores with their special routing resources complicate logic synthesis. QuickLogic tools are schematic based, where Cypress supplies a VHDL development environment which accepts VHDL descriptions, synthesizes them, then maps them to the Cypress devices. Future versions will support partitioned designs.

The CCM2 was originally meant to be fit into silicon using OCTOOLS, a VLSI layout program found resident on the SUN systems in the lab. Simulations on the CCM2 were performed using Mentor Graphics mixed-mode analog and digital Lsim simulator tools. Simulation models were created using $\mathbf{M}$ models [33], which are $C$-language supersets modeling analog and digital systems at the behavioral level. Multiple models were written for the iterative logic unit and the control unit by past classes, then students created separate models of cube calculus operations to be inputs to the simulated hardware. Designs such as state machines are easier to create as behavioral models in comparison to the same design in schematic form.

Once the simulations had been run and verified in their operations, the design to fit it to silicon was planned. After failed attempts to get the needed tools working together correctly, the alternative technology of using programmable logic devices was considered. Due to the lack of proper tools, the absence of VLSI fabrication funding and the availability of the Xilinx tools, the decision to progress in the direction of FPGA design was quite easy.

The CCM2 was targeted for the Xilinx family of Logic Cell Arrays (LCAs) since they were a viable option for the design, and happened to be the available tools at the 
time here at PSU. The tools were brought from Portland Community College by Douglas Hall and quickly acquired by Dr. Marek Perkowski and Dr. Y.C. Jenq for future use in their research. I acquired the tools in the Winter term of 1992 and have been the sole user until the Winter term of 1994 . The tools are now also being used by Dr. Perkowski's students for the design of a control unit for the sonar of Kevin Stanton's PSUBOT, as well as for a Hough transform image processor for the PSUBOT.

My contribution to the CCM2 project entails the improvement of the ILU design, its realization as a reconfigurable FPGA board and the testing of its operation. Due to material resources and practical time constraints, the ILU was held to be a 16-bit machine. The actual hardware realization consists of a wire-wrapped board with two Xilinx devices, each containing four Iterative Cells (ITs) of the ILU design. The design is so regular, that the same design resides in each Xilinx device. This allows the architecture to be scalable by 8 -bit increments, allowing the CCM2 to be targeted toward different applications depending upon the processing requirements needed. The design allows enough internal logic and $\mathrm{I} / \mathrm{O}$ resource capacity to handle many changes to the existing design for future projects, or tailoring the design to a specific application.

To test the design, I have implemented a limited control unit, using an additional GAL device to produce the global signals needed. Inputs to the ILU are controlled by switches, and outputs sent to LED's. Future class projects and Master's Thesis studies will expand upon this design, working on the interface to a host system, instruction registers and bus control logic. Once the CCM2 has been fully realized as an add-on accelerator board, an additional area of targeting the CCM2 to a specific application or task may be implemented.

The following chapters are organized as follows: Chapter II gives an overview of the evolution of FPGAs and gives detailed information concerning the Xilinx family of 
logic cell arrays (LCAs). This will be helpful in the understanding of the devices and the methods of their configuration to future individuals planning on working with these devices and the respective tools. Chapter III presents a subset of cube calculus operations, giving an overview of the algebraic model and the specific operations currently implemented with the presented architecture. Chapter IV introduces the interactive logic unit (ILU) of the CCM2. This section gives a detailed description of the design and functionality of the ILU. Here each block is identified and defined and several examples of cube calculus operations and their execution by the ILU are presented. Chapter V presents the actual realization of the ILU in the Xilinx devices, its control unit (CU) and the results of the hardware implementation and testing. Chapter VI contains the timing analysis of the hardware, a comparison to a software realization and to BDD structures. Finally, Chapter VI contains all of the evaluation of the hardware, and conclusions are drawn and suggestions for improvements to the current design and future development are presented. The Appendix contains a simple tutorial of the Xilinx development tools, with a basic design example, taking a user from the initial schematic design to a fully operational circuit through the use of Xilinx development tools. It also contains design coding, routing and timing results and all technical details pertaining to the CCM2. 


\section{CHAPTER II}

\section{FPGA TECHNOLOGY}

For some time now, combinational network designs have been realized using read only memory (ROM) and programmable logic array (PLA) technologies. A sequential network can be easily constructed with either technology by the addition of D-flip-flops, which do not need multiple inputs such as JK-flip-flops, thus increasing the device size. PALs provide a convenient way of realizing a sequential network since most contain Dflip-flops driven by the programmable logic in the device.

As integrated circuit technology has developed, a wide variety of programmable logic devices (PLDs) have been introduced that can be used to realize both sequential and combinational networks [43]. Typically, PLDs are interconnections of PLAs. The devices have dedicated input, output, and bidirectional I/O pins used appropriately for the programmed design. Although these PLDs are capable of implementing both sequential and combinational networks, they are usually incapable of realizing a complete digital system. This is quite obvious since these logic devices do not have the transistor density to accommodate a typical digital system

Programmable gate arrays (PGAs) are more flexible and versatile than PLDs and are typically used to implement multilevel logic functions. They have the capability of implementing a small digital system on a single Integrated Circuit (IC) chip. A typical PGA is an IC containing an array of identical logic cells with programmable interconnections determined by the configuration which the designer has programmed into the IC. The logic cells, or basic blocks, are a versatile configuration of logic 
elements programmed by the user. Xilinx Inc. introduced the world's first field programmable gate array (FPGA) in 1985 and still remains the market leader in FPGA technologies, which has since become the fastest growing segment of the semiconductor industry.

Xilinx, founded in 1984, based its product design on the idea of combining the logic density and versatility of gate arrays with the time-to-market advantages and off-the-shelf availability of user programmable standard parts [58][59][60]. FPGAs accommodate high density designs, combining an abundance of gates and I/O's with fast system speed.

FPGAs may be designed and verified in a few days, versus several weeks working with typical gate arrays. Since the devices are programmable via software, design modifications may be made in hours saving both design and production costs. For educational purposes, FPGAs are ideal due to their reprogrammability, allowing multiple configurations to be stored externally, downloading revisions to the design when desired. This provides a huge savings since design revisions affecting the FPGA alone take milliseconds with the in-circuit programmability features.

For the Cube Calculus Machine (CCM), there are currently three versions of the architecture on paper that have yet been realized in hardware. The CCM1 was introduced by Luis S. Kida and Dr. Marek Perkowski, then extended to the CCM2 architecture by Dr. Perkowski and his EE510 classes. The CCM2 is the machine which I have concentrated upon and which will be presented in this thesis. Coen Engelbarts further extended the CCM2 architecture to the CCM2.5. The CCM2.5 enables the architecture to operate upon a larger class of cube calculus operations by increasing the number of inputs possible to each Iterative Cell (IT). The CCM3 was placed on paper by Dr. Perkowski with the intent of once again increasing the number of Cube Calculus operations that can be done. With a single FPGA device, The CCM2, CCM2.5 and the CCM3 architectures 
may make use of the device by storing each respective configuration in an external software file. Changing between these architectures on a single device could be realistic by downloading the desired configuration and making little to no external hardware changes to the rest of the hard-wired board.

Once a design is completed and ready for production, a designer using Xilinx FPGAs may convert the design to a hard-wired gate array using the mask and test files produced in the existing FPGA files. The programmable connections are simply removed and replaced with fixed metal connections. By using this approach, designers have the flexibility of working with FPGAs and the ability to fit the final configuration in hardwired gate array devices of smaller die size and cheaper costs.

\section{SELECTING THE RIGHT DEVICE}

Once a designer has decided that a Xilinx FPGA is the correct choice for a particular design, he/she must then make an educated decision in selecting the right device. The designer must identify areas of logic which are suitable for implementation in a Xilinx Logic Cell Array (LCA). Xilinx has several families of FPGA devices varying in architecture, logic capacity, design timing, power consumption and other system features. Once the designer has chosen the family of devices that will work best for this particular design, a specific device within this family must be chosen.

This decision will be based on gate-count, number of $\mathrm{V} / \mathrm{O}$ resources, flip-flops, RAM bits, configurable logic blocks (CLBs) and Macrocells that will not possibly be needed in this design. Ideally, the initial design should consume no more than $70 \%$ of the CLBs and $\mathrm{y} / \mathrm{Os}$ of the targeted device. This allows room for modifications and additions to the design. L/Os are the primary concern when selecting a device since they are the limiting factor of the design and determine the package size of the device. Once a device from a 
particular package has been chosen, the designer has a choice between surface mount and through-hole package types, which accommodate a variety of I/O counts, body types and temperature ratings.

For the design of the ILU for the CCM2, we have chosen the XC3090 175-pin PGAgrid device. This device was chosen due to its logic density, number of I/Os and the current programming tools that we have here at Portland State University. It has the logic density of the XC3000 family, with the ability to accompany an 8-IT, 16-bit ILU. The regularity of the design allows a 32-bit and 64-bit machine to be realized by daisychaining additional devices. The following sections consider the architecture and configuration, and will be targeted towards the XC3000 family and our specific device.

\section{LCA ARCHITECTURE}

The Xilinx LCA families of FPGA devices provide a fully flexible userprogrammable CMOS gate array. The XC3000 LCA family provides a group of highperformance, high-density, digital integrated circuits [58][59][60]. The devices are quite regular, flexible and extendible in their architecture. The user-programmed array architecture is composed of a configuration program store plus an internal core of configurable logic blocks (CLBs), a perimeter of $\mathrm{L} / \mathrm{O}$ blocks (IOBs) and a grid of programmable interconnect resources.

\section{Configuration Memory}

The LCA functions are established by a configuration program that is loaded into an internal, distributed array of static configuration memory cells designed with high reliability and noise immunity. The configuration program is loaded into the LCA device at power-up and may be reloaded on command. The configuration bit-stream used to 
configure the LCA device is generated through the Xilinx XACT development system. The basic memory cell consists of two CMOS inverters and a pass transistor used for writing and reading cell data. The cell is only written during configuration and read only during read-back. During normal operation the cell provides continuous control and does not affect cell stability. There are several methods for loading the configuration data. The method I have chosen will be discussed in Chapter V.

\section{$\underline{\text { I/O Block }}$}

The configurable IOBs provide a programmable interface between the internal logic array of CLBs and the device package pins. Each IOB provides both registered and direct input paths, and a programmable three-state output buffer driven by a direct output signal. Also included are input clamping diodes to protect from electrostatic damage, and circuitry to protect against latch-up due to input currents. The input buffer provides threshold detection to translate externally applied signals to internal logic levels. The threshold can be programmed to be compatible with either TTL (1.4V) or CMOS (2.2V) levels. The buffered input signal drives the data input of a storage element, which may be configured as either a flip-flop or a latch. Output buffers provide CMOS-compatible 4-ma source-or-sink drive for high fan-out CMOS or TTL-compatible signal levels. Each IOB has configuration program bits controlling additional features such as output registers, logic signal inversion and 3-state and slew-rate control of the output.

\section{Configurable Logic Block}

A CLB is basic block, a versatile configuration of logic elements which can be programmed by the user. A CLB is a RAM-based cell that can implement any singleoutput logic function of up to five input variables, or any two-output logic function of up to five input variables with each output depending on at most four input variables [14]. 
The array of CLBs constitutes the core of the LCA device, providing the functional elements from which the user's logic is constructed. The CLBs are arranged in a matrix within the perimeter of IOBs. For example, the XC3090 has 320 CLBs in a 16-row by 20-column array surrounded by a perimeter of 144 user I/Os. The operation and connectivity of each block is programmed by the user with the XACT development system which compiles the configuration data to be loaded to the internal configuration memory. A standard CLB in the XC3000 LCA family has a table look-up (TLU) block structure with a combinatorial logic section, two flip-flops, and an internal control section (Figure 1).

There are five logic inputs $[\mathrm{A}, \mathrm{B}, \mathrm{C}, \mathrm{D}, \mathrm{E}]$; a common clock input $[\mathrm{K}]$; an asynchronous direct reset input [RD]; and an enable clock [EC]. All inputs may be driven by the interconnect resources adjacent to the blocks, connecting IOBs and other CLBs. Each CLB also has two outputs $[\mathrm{X}$ and $\mathrm{Y}]$ which may drive interconnect networks connected to other CLBs and IOBs. Data input for either flip-flop within a CLB is supplied from the function $\mathrm{F}$ or $\mathrm{G}$ outputs of the combinatorial logic, or the block input, data-in [DI]. All flip-flops are reset by the active-Low chip input, $\overline{\text { RESET}}$, or during the configuration process.

The combinatorial logic portion of the CLB uses a $32 \times 1$ look-up table to implement Boolean functions. Variables selected from the five logic inputs and two internal block flip-flops are used as table address inputs. This technique can generate several combinatorial logic options. Option F generates any function of five variables: A, D, E, and two choices out of B, C, QX, QY. Option FG generates two independent logic functions of up to four variables each. One variable, A, must be common to both functions, with the second and third variables chosen from $\mathrm{B}, \mathrm{C}, \mathrm{QX}$, or QY, and the fourth option being either D or E. The final option FGM allows the variable $\mathrm{E}$ to select 
between two functions of four variables. Both functions have common inputs $A$ and $D$, and may choose from $\mathrm{B}, \mathrm{C}, \mathrm{QX}$ and $\mathrm{QY}$ for the remaining two variables. This allows the implementation of 6 and 7 variable functions in one CLB.

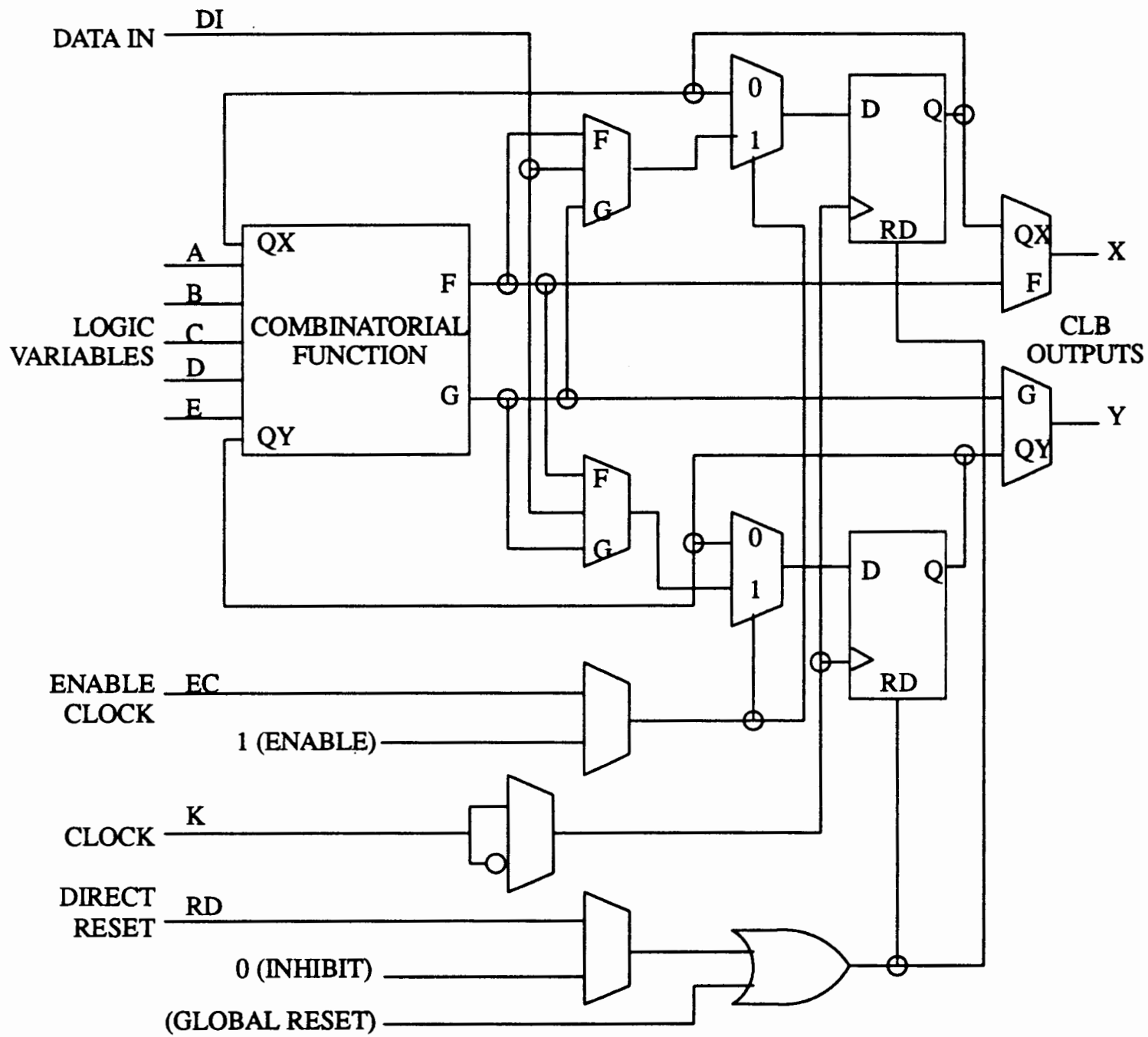

Figure 1. XC3000 configurable logic block.

For example, if the number of inputs is five, and $f_{1}=a b c d e g$ and $f_{2}=a b c+\bar{b} d e+\bar{a} \bar{e}+\bar{c} \bar{d}$, both functions have 6 and 10 literals respectively. Function $f_{1}$ requires two CLB's in its optimum implementation, since each CLB will accept a maximum of 5 inputs. Function $f_{2}$ can be implemented in only one CLB since it is a 
function of 5 variables. Thus, the objective function has to do more with the number of inputs than with the actual logic that the function realizes. Current interest is being focused on reducing the number of configurable logic blocks used within the fixed architecture of the programmable gate array. The total number of CLB's may be reduced by finding pairs of lookup tables that fit inside a single CLB. Finding the number of such pairs can be restated as a maximum cardinality matching problem [18].

\section{Programmable Interconnect}

Programmable-interconnection resources in the LCA provide routing paths for connecting inputs and outputs of the IOBs and CLBs to logic networks. Pass transistors controlled by a single configuration bit form programmable interconnect points (PIPs) and switching matrices used to implement the necessary connections between the twolayer grid of metal segments and block pins. The XACT development system provides automatic routing of the segments as well as interactive routing for design optimization. There are three types of metal resources to accommodate various network interconnect requirements: general purpose interconnect; direct connection; and longlines.

General Purpose Interconnect. General purpose interconnect consists of five horizontal and five vertical metal segments located between the rows and columns of logic and IOBs. Switching matrices join the segments allowing programmed interconnections between the metal grid segments and the adjoining rows and columns. Special bidirectional (BIDI) buffers are provided within the general interconnect providing periodic signal isolation and restoration for improved performance of lengthy nets. The buffers are able to propagate signals in either direction on a given segment and are automatically defined by the XACT development system, based on the location of the interconnection network source. 
Direct Interconnect. Direct interconnects route signals from block to block, exhibiting minimal interconnect propagation and using no general interconnect resources. They provide the most efficient implementation of networks between adjacent CLBs or IOBs. Direct interconnect should be used to maximize the speed of high-performance portions of logic.

Longlines. Longlines are intended primarily for signals that must travel a long distance, or must have minimum skew among multiple destinations. The longlines run vertically and horizontally the height or width of the interconnect area, bypassing the switch matrices. There are two horizontal longlines per interconnect row and three vertical longlines per interconnect column. Two additional longlines are located adjacent to the outer sets of switching matrices. The longlines can be driven by logic blocks or IOB outputs. 


\section{PROGRAMMING THE LCA}

When selecting an LCA device for a design, the user needs to compare the different LCA families and device types. However, once the device has been selected, the user needs to carefully plan the design to optimally fit the device. Logic areas suitable for implementation in an LCA must be identified and partitioned into clusters of basic logic elements representing configurable logic blocks and I/O blocks. A design file must then be created using the XACT development system for each LCA. Once the design file is complete, it is compiled into a configuration program determining the function of the chosen LCA. Using XACT, the configuration program can also be translated into formatted files compatible with standard EPROM programming equipment. Using this method allows an EPROM to store the LCA configured program. The following sections describe the general design flow and configuration phases for a typical LCA design.

\section{$\underline{\text { Design Flow }}$}

The design flow consists of the iterative entry, implementation and verification processes of the design. Due to an LCAs re-programmability, design iterations through the edit-implement-verify loop can be done in minutes. Figure 2 shows the design flow from the design entry to the final design configuration ready to be downloaded.

Design Entry. Design entry is the process of describing a logic function in electronic form. Designs may be entered through schematic capture, boolean equations or state machine languages. Any or all of these methods of entry may be used in the same design. Each schematic, equation or description language specification is translated into separate Xilinx Netlist Files (XNFs), then merged in a single design file, ready for conversion into an LCA file. 
Design Implementation. The conversion of the XNF into an LCA design file is a part of the design implementation. The LCA contains definitions of IOBs and CLBs with interconnection details for routed designs. Many options are available for the conversion, allowing automatic translation, partitioning, placement and routing of the design. Once the XNF has been translated into an LCA file, the LCA may then be viewed by the XACT development system.

Within the XACT design editor (XDE), the design may be edited, altering and adding CLBs, IOBs and the routing and connections between the two. At this point, a design may also be created through interactive design methodologies, bypassing the design entry stages and partitioning and routing the design by hand. The design is now ready to be verified and downloaded to the LCA device.

Design Verification. There are many methods of verifying the design depending on the needs of the user. Logic simulation or actual in-circuit emulation provides functional verification while timing analysis allows verification of critical timing paths under worstcase conditions. Post route timing information is created during the file conversion process when converting an LCA file to an XNF. Time dependent behavior of the programmed LCA in response to input stimuli may be found with an additional package XNF2SILOS.

The LCA design is then downloaded from XACT through a generated bit-stream file describing the logic programming configuration. An in-circuit verifier XACTOR allows bit-stream downloading and retrieval of a "snapshot" of the I/O and CLB flip-flop logic states with stationary inputs. Chapter VI contains a brief tutorial based upon the Xilinx 2064 which follows through the complete design flow beginning with the design entry and finishing with an operational LCA design file. 
Configuring the Device

The configuration of the device is user-selectable depending on how the device is intended for use. There are three phases of the configuration sequence: initialization, configuration and user-operation. 


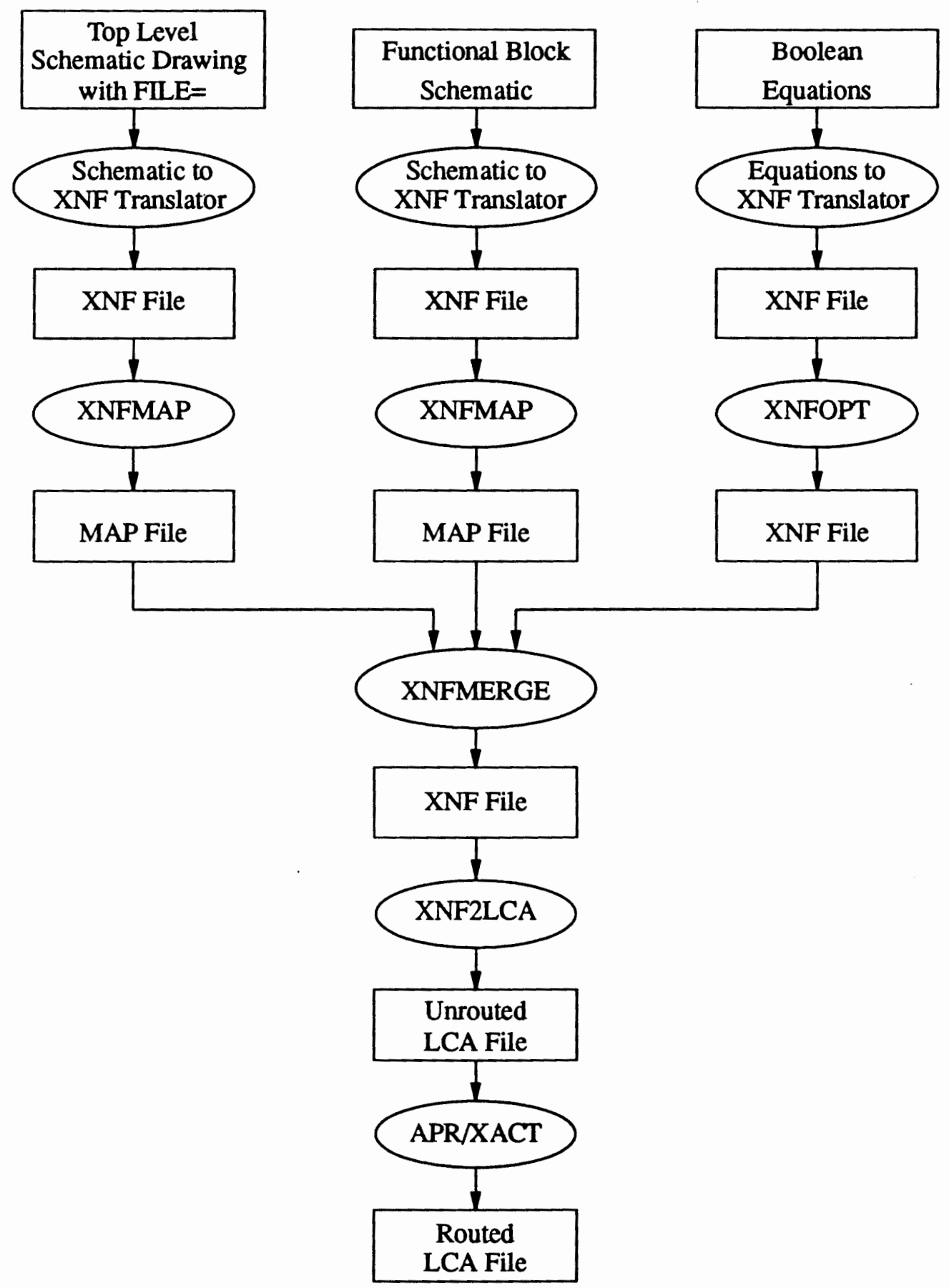

Figure 2. Design flow for merging functional blocks. 
Initialization Phase. After an initial power-up delay, the LCA awakens in the initialization state in which all internal configuration memory is cleared and all internal user-definable logic is held at an idle state. Once complete, the LCA checks the RESET pin for its present logic level. When a valid logic "1" is detected, the device enters the configuration state.

Configuration Phase. Configuration data defining the design's function and the interconnection of the logic within the LCA is loaded from an external storage at powerup and after a reprogram signal. Several modes of automatic and controlled loading of the required data are available: master-serial, master-low, master-high, peripheral and slave. The configuration program is loaded into the LCA as if it were a shift register with a format like a serial bit-stream. The configuration program contains a bit field which indicates its length. When the correct number of bits have been entered, the D/P opendrain-output goes high, indicating completion of the configuration. Once configuration has started, it must be completed or aborted and restarted. Partial configurations are not possible.

User-Operation Phase. In this state, all configuration has been completed successfully, and the LCA performs the user specified logic functions. 


\section{CHAPTER III}

\section{CUBE CALCULUS OPERATIONS}

A Boolean algebra $B$ is a set of elements $a, b, c, \ldots$, together with two binary operations, + and $\cdot$, satisfying the commutative and associative laws and are mutually distributive [31]. B contains an upper and lower bound of 1 and 0 respectively. In the CCM, we use a Boolean based algebraic model that operates upon multiple-valued inputs and returns a single-valued output based upon the operation on those input values.

The main concepts of the cube calculus algebraic model are those of a cube and an array of cubes [12][13][17][30][42][44][51][52][53]. The cube can represent either a product of literals, a sum of literals, or an exclusive sum of literals. For binary functions, a literal is a binary variable with or without negation. For any subset $S_{i} \subseteq P_{i}, X_{i} S_{i}$ is a literal of $X_{i}$ representing the function such that:

$$
X_{i}^{S_{i}}= \begin{cases}0 & \text { if } X_{i} \& S_{i}: X^{0}=\bar{X} \\ 1 & \text { if } X_{i} \in S_{i}: X^{1}=X\end{cases}
$$

A binary function $f(a, b, c, d)$ where $f=a c+b \overline{c d}$, is represented in a Karnaugh map in Figure 3. 


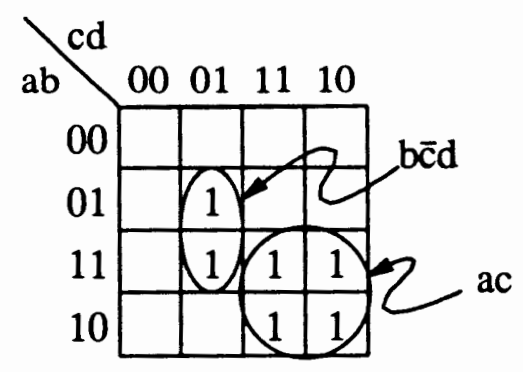

Figure 3. Binary function $f=a c+b \bar{c}$.

For a multiple-valued input, two-valued output, incompletely specified switching function $f$, a mapping of $f\left(X_{1}, X_{2}, \cdots, X_{N}\right): P_{1} \times P_{2} \times \cdots P_{N} \rightarrow B$, where $X_{i}$ is a multiple-valued variable, $P_{i}=\left\{0,1, \ldots, p_{i}-1\right\}$ is a set of true values that this variable may assume, and $\mathbf{B}=\{0,1,-\},(-$ denotes a don't care value $)$ [13][25][30][38][41][42][53]. $\mathrm{N}$ denotes the number of variables (literals, positions) of a cube. For a four-valued input logic of $X^{\{0,1,3\}}=1$, if $X \in 0,1,3$ which means, $X^{\{0,1,3\}}=1$, if $(X=0)$ or $(X=1)$ or $(X=3)$. Otherwise: $X^{\{0,1,3\}}=0$. For $(X=2), X^{\{0,1,3\}}=0$. $A$ multiple-valued function $f(A, B)$, where $f=A^{0} B^{1} \cdot A^{1} B^{1}+A^{1} B^{2} \cdot A^{2} B^{2}$ $=A^{01} B^{1}+A^{12} B^{2}$, can be represented in a Karnaugh map where each cell is a combination of variables, as seen in Figure 4.

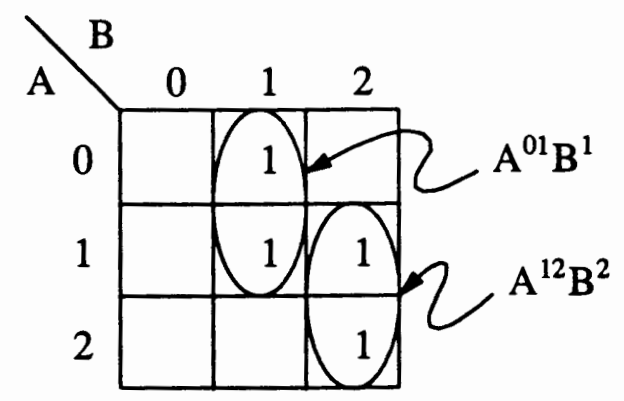

Figure 4. Mult-valued function $f=A^{01} B^{1}+A^{12} B^{2}$.

A product of literals, $X_{1} S_{1} X_{2}^{S_{2}} \ldots X_{n}^{S_{N}}$, is referred to as a product term, or product 
for short and is represented as a cube. A product term that includes literals for all function variables $X_{1}, X_{2}, \ldots, X_{N}$ is called a full term. Any literal of the form $X_{i}{ }_{i}$ is equal to 1 , thus $\mathrm{X}_{\mathrm{i}}^{\mathrm{P}_{\mathrm{i}}} \mathrm{X}_{\mathrm{j}} \mathrm{S}_{\mathrm{j}}$ can be written as $\mathrm{X}_{\mathrm{j}} \mathrm{S}_{j}$. A sum of products is referred to as a sum-of-products expression (SOPE), while a product of sums is called a product-of-sums expression (POSE). An EXOR of products will be called an Exclusive Sum of Products Form (ESOP), and a product of EXORs will be called a Product of Exclusive Sums expression (POES) [17][24][39][44][49]. SOPE, POSE, ESOP and POES are all represented as arrays of cubes. Products of SOPEs (PSOPEs) may also be used, and are represented as arrays of arrays of cubes.

Switching functions with multiple-valued inputs, two-valued outputs, find several applications in logic design, pattern recognition, and other areas. In logic design, they are primarily used for the minimization of PLAs that have decoders on the inputs, minimization of multi-output binary functions and state assignment of Finite State Machines (FSMs). A Programmable Logic Array (PLA) with r-bit decoders directly realizes a SOPE of a $2^{r}$-valued input, two-valued output function.

$2 \mathrm{n}$ is denoted to be the number of bits of a word (register) that contains a cube in positional notation. To focus our considerations, we assume $2 n=32$ bits. We can have as many as $n=16$ binary variables in a cube. The encoding is as follows: $x-10, \bar{x}-01$, don't care (often denoted by $X$ ) -11 , and contradiction -00 . In this notation, the intersection of two cubes representing products of literals simply corresponds to a bit-bybit product of the respective words. For instance, assume 4 binary variables, (a, b, c, d), with a product of:

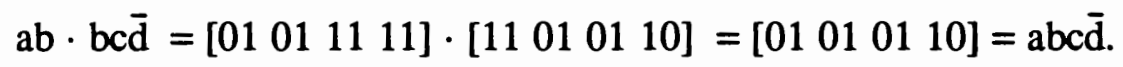

When the opposite literals are multiplied, the pair 00 is created from the bit-by-bit product and is detected in the next stages: 
$\mathrm{ab} \cdot \mathrm{a} \bar{b}=\left[\begin{array}{llll}01 & 01 & 11 & 11\end{array}\right] \cdot\left[\begin{array}{llll}01 & 10 & 11 & 11\end{array}\right]=\left[\begin{array}{llll}01 & 0 & 11 & 11\end{array}\right]=$ contradiction.

The contradiction is detected and signalized by the machine. For multi-valued input logic, the positional notation takes for a variable as many bits as this variable can take values. For instance, a 4-valued variable takes 4 bits. Assuming the first variable of 4 values and the second variable of 6 values, the product $X_{1}^{A_{1}} X_{2}^{A_{2}}=X_{1}^{0,1,2} X_{2}^{1,3}$ is represented as a cube $A=\left[A_{1}, A_{2}\right]==[1110010100]$. Assuming 4-valued variables and $2 n=32$, one has 8 four-valued variables in a cube. It is assumed in the CCM architecture that each variable can have an arbitrary even number of values.

\section{SEQUENTIAL OPERATIONS}

The architecture of the CCM2 has been constructed to efficiently execute the family of sequential cube calculus operations. Each sequential cube calculus operation can be described by the following pattern using the argument cubes $A$ and $B$ as:

$$
\begin{aligned}
& \mathrm{A}=\mathrm{X}_{0}^{\mathrm{A}_{0}} \mathrm{X}_{1}^{\mathrm{A}_{1}} \cdots \mathrm{X}_{\mathrm{N}-1}^{\mathrm{A}_{\mathrm{N}},}, \\
& \mathrm{B}=\mathrm{X}_{0}^{\mathrm{B}_{0}} \mathrm{X}_{1}^{\mathrm{B}_{1}} \cdots \mathrm{X}_{\mathrm{N}-1}^{\mathrm{B}_{\mathrm{N}-1},},
\end{aligned}
$$

where $A_{j}=\left(a_{0}, a_{1}, \cdots, a_{k_{j}-1}\right)$, and for each bit $a_{m}$ from $A_{j}, a_{m} \in\{0,1\}$. Similarly, $B_{j}=\left(b_{0}, b_{1}, \cdots, b_{k_{j}-1}\right), b_{m} \in\{0,1\}$.

Each variable $X_{j}$ can take one of the values of 0 through $k_{j}-1$. $A_{j}$ specifies the set of values of $X_{j}$ for which $x_{j}^{A_{j}}$ is true. Presence of " 1 " in $A_{j}$ in position $m$ (that is, $a_{m}=1$ ) means, that $x_{j}^{A_{j}}$ is true whenever $X_{j}=m$. Presence of " 0 " (that is, $a_{m}=0$ ) means that $X_{j}^{A_{j}}$ is false for $X_{j}=m$. Each bit of $A_{j}$ can be either " 0 " or " 1 " independently of other bits. For binary logic, $X_{j}^{00}$ is false for any value of $X_{j} ; X_{j}^{10}$ is true only for $X_{j}=1 ; X_{j}^{01}$ is true 
only for $X_{j}=0 ; X_{j}^{11}$ is true for both $X_{j}=0$ and $X_{j}=1$.

The array of resultant cubes produced by the sequential cube calculus operation (op) can be described as follows:

$$
\begin{gathered}
A(o p) B= \\
\left\{C_{k} \mid C_{k}=X_{1}^{\operatorname{aft}\left(A_{1}, B_{1}\right)} \cdots X_{k-1}^{\operatorname{aft}\left(A_{k-1}, B_{k-1}\right)} X_{k}^{a c t\left(A_{k}, B_{k}\right)} X_{k+1}^{\operatorname{bef}\left(A_{k+1}, B_{k+1}\right)} \cdots X_{N}^{\operatorname{bef}\left(A_{N}, B_{N}\right)}\right\} \\
\left.=\left\{C_{i} \mid C_{i}=\prod_{j=1}^{N} X_{i}^{c_{j}}\right\}, \quad c_{j}=\left(c_{0}, c_{1}, \cdots, c_{k_{i}-1}\right), i \in(1, \ldots, m)\right),
\end{gathered}
$$

where if $m$ is the number of pairs of literals for which relation rel is true, and $i \in(1 . . m)$ is the number of $i$-th such pair of $A_{k}$ and $B_{k}$, for which the relation rel is true.

An important property of functions before (bef for short), active (act) and after (aft) is that they are bit-wise functions, that is, bit $c_{i}$ of the result of each of these functions is dependent only on bits $a_{i}$ and $b_{i}$ of the arguments and therefore the function is defined for cubes of arbitrary size. Values returned by the rel function are determined as an OR or AND combination (depending on the type of the cube calculus operation) of the partial relations for single bits. Each partial relation is determined in bit-wise fashion, that is, it depends only on the values of the corresponding bits of the arguments. Therefore the rel function is also defined for cubes of arbitrary size.

Pairs of literals for which rel is true are called specific literals. Variables for which literals are specific will be called specific variables, and their corresponding position will be called the specific position. As in the above description, the first resultant cube for a sequential cube calculus operation is produced for the first specific literal selected as the active one. Later, the next specific literal is selected as the active one, and the next resultant cube is produced. This procedure is repeated until the last specific literal has been selected as the active one. For a given resultant cube all the literals with numbers 
less than the number of the specific literal are of the after type, all the literals with numbers greater than the number of the specific literal are of the before type.

\section{Crosslink}

The crosslink operation on cubes A and B creates an array of cubes defined as follows:

$$
\begin{gathered}
A \square B= \\
\left\{X_{1}^{B_{1}} \cdots X_{i-1}^{B_{i-1}} X_{i}^{A_{i} \cup B_{i}} X_{i+1}^{A_{i+1}} \cdots X_{N}^{A_{N}}\right. \\
\text { I for such } \left.i=1, \ldots, N \text {, that } A_{i} \cap B_{i}=\varnothing\right\}
\end{gathered}
$$

This is a basic operation used in minimization of Generalized Reed Muller forms, ESOPs, and other forms based on EXOR gates [17][24][39][44][49]. The crosslink operation on cubes A and B is a representation of all minterms of cubes A and B by an EXOR of multiple cubes. All minterms of A and B are included in an odd number of cubes, with all other minterms included in an even number of cubes, or in no cube at all. Since $1 \oplus 1=0$ and $1 \oplus 1 \oplus 1=1$, this is a valid representation of cubes A and B. It can be seen in the above equation that the crosslink operation is not symmetrical, thus $A \square B \neq B \square A$. When the order of the argument cubes is changed, another set of resultant cubes will be generated, but the number of cubes and the size of the cubes will remain the same [13][30].

The crosslink operation can only be applied to two cubes when: the cubes are specified for the same variables (that is, the literals which are true for all possible values are in the same positions); the two cubes are of the same degree (the degree of a cube is the number of literals in the cube that are not equal to one). The number of resultant 
cubes is equal to the distance of the two operand cubes. The crosslink operation is not always an ideal operation if using it directly for circuit minimization, since the greater the distance of the two input cubes, the greater the number of output cubes result. An example is illustrated in Figure 5 with the crosslinking of input cubes A and B.

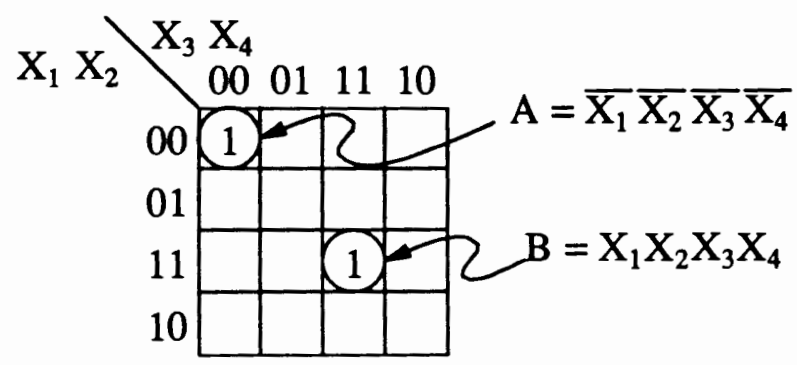

Figure 5. Binary cubes A and B to be crosslinked.

The result of the crosslink operation upon cubes A and B may be seen in Figure 6. Here, $\overline{X_{1}} \overline{X_{2}} \overline{X_{3}} \overline{X_{4}} \square X_{1} X_{2} X_{3} X_{4}=\overline{X_{1}} \overline{X_{2}} \overline{X_{3}}+\overline{X_{1}} \overline{X_{3}} X_{4}+\overline{X_{1}} X_{2} X_{4}+X_{2} X_{3} X_{4}$.

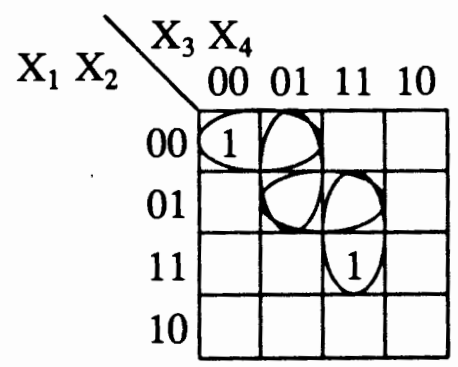

Figure 6. Four resultant cubes from the crosslink function.

\section{Nondisjoint Sharp}

The nondisjoint sharp (sharp) on cubes A and B is defined as follows:

$$
\mathrm{A} \# \mathrm{~B}=\left\{\begin{array}{cc}
\mathrm{A} & \text { when } \mathrm{A} \cap \mathrm{B}=\phi \\
\phi & \text { when } \mathrm{B} \supseteq \mathrm{A} \\
\mathrm{A} \#_{\text {basic }} \mathrm{B} & \text { otherwise }
\end{array}\right.
$$


where $A \#_{\text {basic }} B$ is defined as follows:

$$
\begin{gathered}
A \#_{\text {basic }} B= \\
\left\{X_{1}^{A_{1}} \cdots X_{i-1}^{A_{i-1}} X_{i}^{\neg B_{i} \cap A_{i}} X_{i+1}^{A_{i+1}} \cdots X_{N}^{A_{N}}\right. \\
\text { I for such } \left.i=1, \ldots, N \text {, that } \neg\left(B_{i} \supseteq A_{i}\right)\right\}
\end{gathered}
$$

This is a basic operation used in minimization of PLAs, tautology, complementation, and all other general purpose logic operations. The nondisjoint sharp of cubes A and B is the set of largest cubes that are included in cube A, but not in cube B. The result of the nondisjoint sharp is a nonsymmetrical, unique SOPE. By $B_{i} \supseteq A_{i}$ we denote the relation of set inclusion, that is, that set $B_{i}$ includes set $A_{i}$, in positional notation: $\forall j=0, \ldots, p_{i-1}, \quad\left[B_{i}^{j} \geq A_{i}^{j}\right]$. Formula $\neg\left(B_{i} \supseteq A_{i}\right)$ is the predicate that is true when the relation $B_{i} \supseteq A_{i}$ is not satisfied. $B y B \supseteq A$ we denote positional cube inclusion, that is, $\mathrm{B} \supseteq \mathrm{A} \Leftrightarrow \forall \mathrm{i}=1, \ldots, N\left[\mathrm{~B}_{\mathrm{i}} \supseteq \mathrm{A}_{\mathrm{i}}\right]$. Figure 7 shows the nondisjoint sharp of input cubes $A=U^{1234} \cdot Z_{12}$ and $B=U^{23} \cdot Z^{23}$ in Karnaugh Map representation. Two resultant cubes are produced by the nondisjoint sharp operation and may be seen in Figure 8 . 


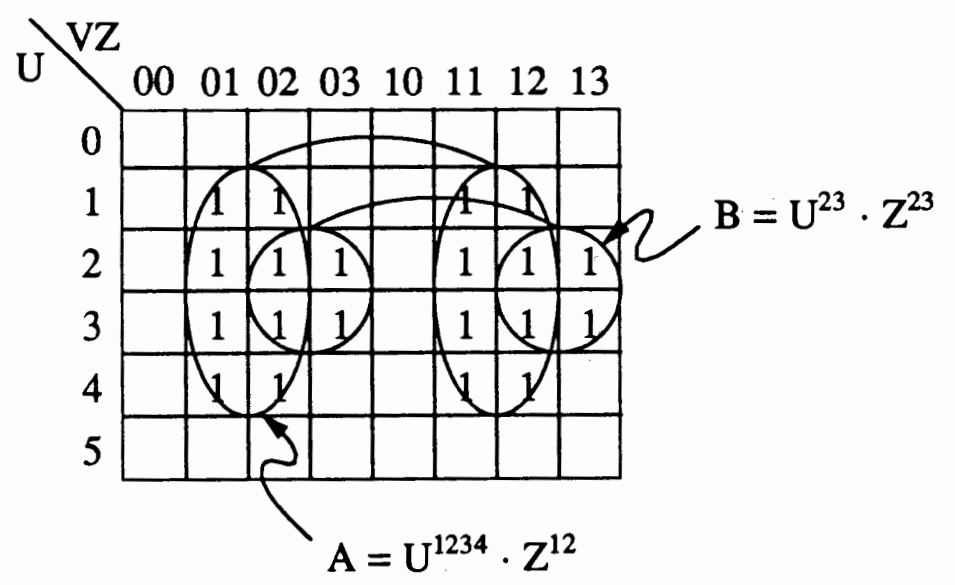

Figure 7. Input cubes for the sharp operation $U^{1234} \cdot Z^{12} \# U^{23} \cdot Z^{23}$.

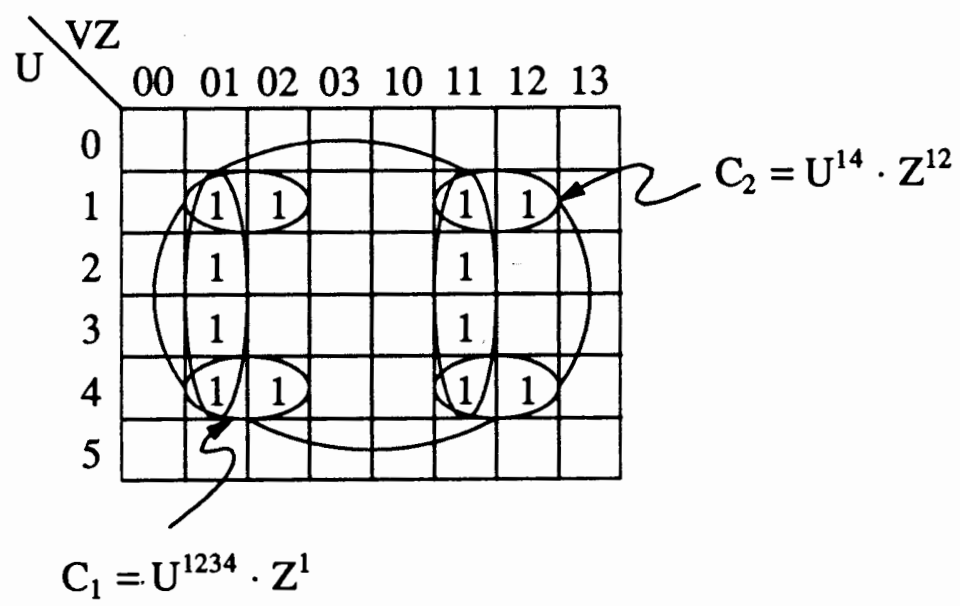

Figure 8. Nondisjoint resultant cubes $\mathrm{U}^{14} \cdot \mathrm{Z}^{12}+\mathrm{U}^{1234} \cdot \mathrm{Z}^{1}$.

Disjoint Sharp

The disjoint sharp (sharp) on cubes A and B is defined as follows:

$$
A \# d B=\left\{\begin{array}{cc}
A & \text { when } A \cap B=\phi \\
\phi & \text { when } B \supseteq A \\
A \# d_{\text {basic }} B & \text { otherwise }
\end{array}\right.
$$

where $A \#_{d_{\text {basic }}} B$ is defined as follows:

$$
\begin{gathered}
A \# d_{\text {basic }} B= \\
\left\{X_{1}^{A_{1} \cap B_{1}} \cdots X_{i-1}^{A_{i-1} \cap B_{i-1}} X_{i}^{\neg B_{i} \cap A_{i}} X_{i+1}^{A_{i+1}} \cdots X_{N}^{A_{N}}\right.
\end{gathered}
$$


I for such $i=1, \ldots, N$, that $\left.\neg\left(B_{i} \supseteq A_{i}\right)\right\}$

The disjoint sharp is basically the same function as the nondisjoint sharp with the same resultant set of minterms, but now represented by an SOPE of which the products each cover a separate group of minterms. If the cubes A and B are disjoint, or mutually exclusive, they do not have a common element, that is, $\mathrm{A} \cdot \mathrm{B}=\phi[15][17][31]$. There is more than one way to represent the minterms that result from a disjoint sharp by an SOPE, dependent on the order in which the variables or the argument cubes $\mathrm{A}$ and $\mathrm{B}$ are given. Figure 9 shows the resultant cubes for the function $U^{12345} \cdot d \# U^{23} \cdot Z^{23}$.

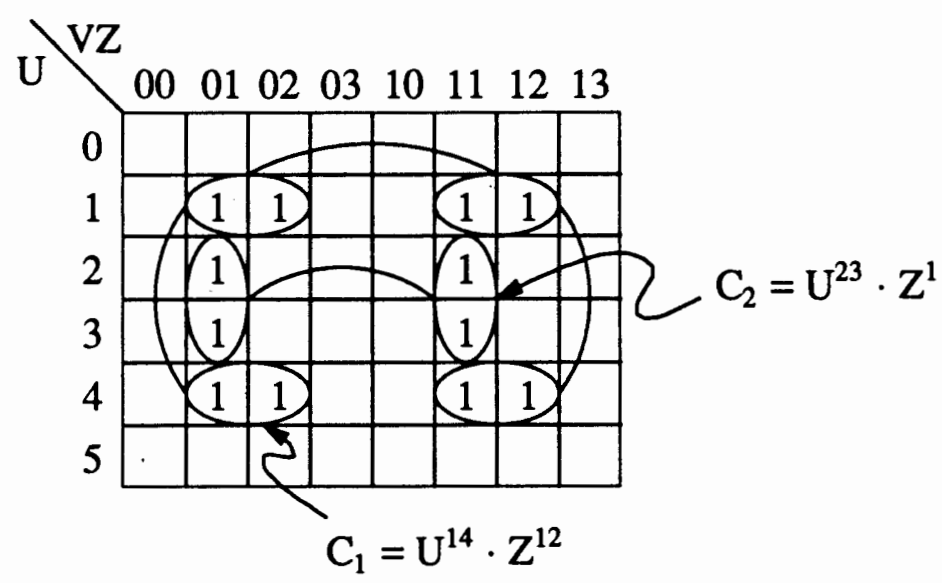

Figure 9. Disjoint sharp resultant cubes $\mathrm{U}^{14} \cdot \mathrm{Z}^{12}+\mathrm{U}^{23} \cdot \mathrm{Z}^{1}$.

\section{Symmetric Consensus}

The symmetric consensus of two cubes $\mathrm{A}$ and $\mathrm{B}$, is the largest minimal cube that covers parts of the two adjacent cubes and is included in both of them. The operation of symmetric consensus is as follows:

$$
A * s B=\left\{\begin{array}{cc}
A \cap B & \text { when } \operatorname{distance}(A, B)=0 \\
\phi & \text { when } \operatorname{distance}(A, B)>1 \\
A^{*} s_{\text {basic }} B & \text { otherwise }
\end{array}\right.
$$


where $A *^{*} s_{\text {basic }} \mathrm{B}$ is defined as follows:

$$
\begin{gathered}
A * s B= \\
\left\{X_{1}^{A_{1} \cap B_{1}} \cdots X_{i-1}^{A_{i-1} \cap B_{i-1}}{X_{i}^{A_{i}} \cup B_{i}}_{X_{i+1}}^{A_{i+1} \cap B_{i+1}} \cdots X_{N}^{A_{N} \cap B_{N}} \text { | for } i=1, \ldots, N\right\}
\end{gathered}
$$

Figure 10 shows the symmetric consensus of input cubes A and B in Karnaugh map representation.

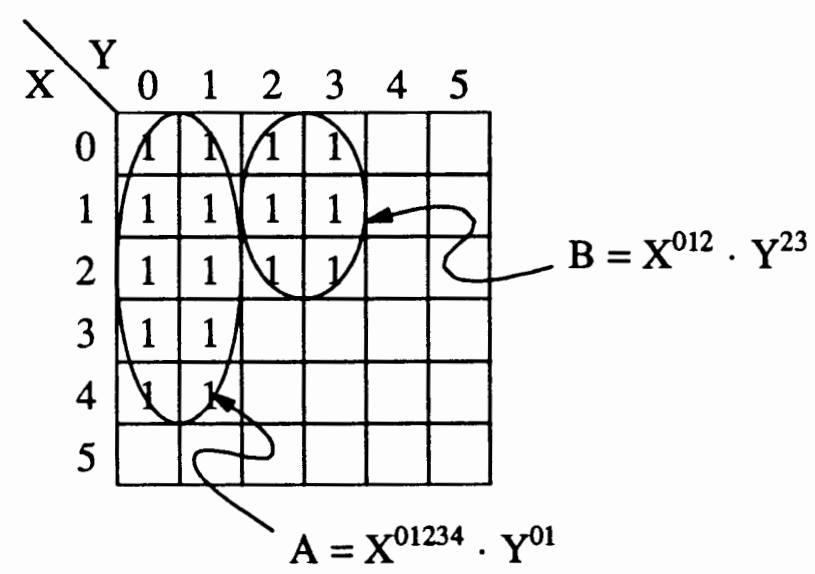

Figure 10. Symmetric consensus of $A * s B=X^{01234} \cdot Y^{01} * s X^{012} \cdot Y^{23}$.

In Figure 11, the single resultant cube from the symmetric consensus operation on cubes $A$ and $B$ can be seen. 


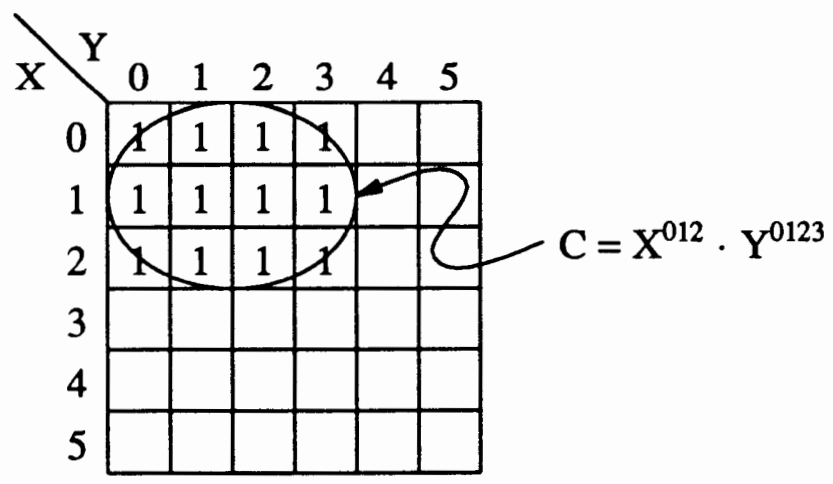

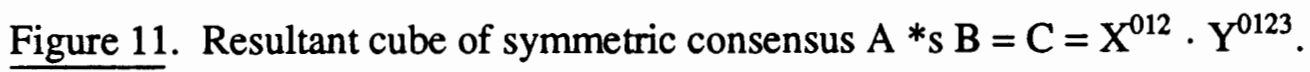

\section{Asymmetric Consensus}

The asymmetric consensus is similar to the symmetric consensus, but the operation $\neg\left(\mathrm{B}_{\mathrm{i}} \supseteq \mathrm{A}_{\mathrm{i}}\right)$ is additionally checked:

$$
\begin{gathered}
A * a B= \\
\left\{X_{1} A_{1} \cap B_{1} \ldots X_{i-1}^{A_{i-1} \cap B_{i-1}} X_{i}^{A_{i} \cup B_{i}} X_{i+1} A_{i+1} \cap B_{i+1} \ldots X_{N}^{A_{N} \cap B_{N}}\right. \\
\text { I for such } \left.i=1, \ldots, N \text {, that } \neg\left(B_{i} \supseteq A_{i}\right)\right\}
\end{gathered}
$$

Figure 12 shows the asymmetric consensus of input cubes A and B in Karnaugh map representation. 


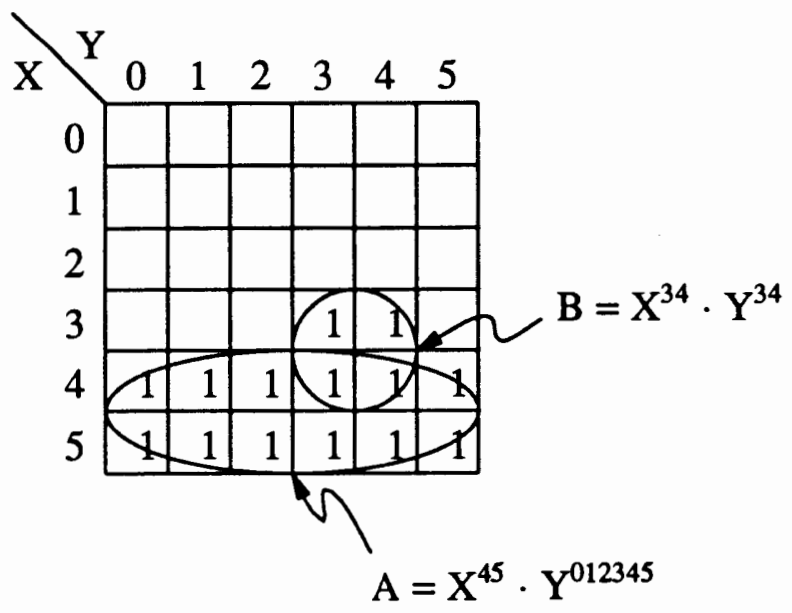

Figure 12. Asymmetric consensus $\mathrm{A} * \mathrm{a} \mathrm{B}=\mathrm{X}^{45} \mathrm{Y}^{012345} * \mathrm{a} \mathrm{X}^{34} \mathrm{Y}^{34}$.

In Figure 13, the single resultant cube from the asymmetric consensus operation on cubes $A$ and $B$ can be seen.

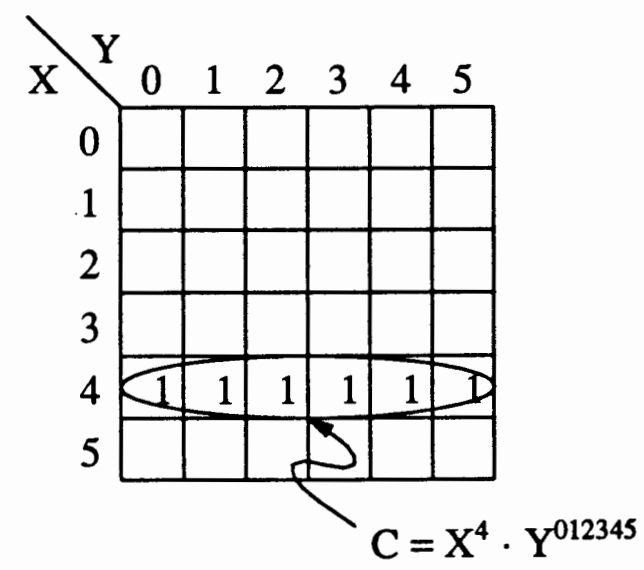

Figure 13. Resultant cube of $A * a B=C=X^{4} Y^{012345}$.

Let us observe that the symmetric consensus and the asymmetric consensus both have the same basic formula, but the conditions that need to be satisfied, are different. The symmetric consensus uses $\mathrm{A} *_{\text {basic }} \mathrm{B}$, which does not check any condition. The 
standard consensus uses the same formula, but only when condition distance $(A, B)=1$ is satisfied. This condition will be called the prerelation. The asymmetric consensus uses an altered version of $\mathrm{A}{ }_{\text {basic }} \mathrm{B}$, which applies a set union for every pair of literals for which the relation $\neg\left(B_{i} \supseteq A_{i}\right)$ is satisfied.

For binary logic, the consensus operations have the same resultant cube. For multivalued logic, the result of the asymmetric consensus can be different from the standard consensus. This is the case, when distance $(A, B)=0$ and there is no such $i$, that $\neg\left(B_{i} \supseteq A_{i}\right)$. In this case $A * B=A \quad B, A * s B=A *$ basic $B$ and $A * a B=\varnothing$.

It should be recognized in the above formulas that all sequential operations have the same basic structure. Each resultant cube has one specific literal which is the literal ${ }_{i}$. The operations that have to be performed on other literals depend on the position with respect to this specific literal. A resultant cube will not exist for all values of $i$, and should be created only when some relations on literal values are satisfied. Generally, the specific literal has to satisfy a certain relation for all given operations. Essential similarities between the definitions of sharp and crosslink can distinguish four aspects:

The relation on literals that must be satisfied in position $\mathrm{i}$ to create a cube (like $\neg\left(B_{i} \supseteq A_{i}\right)$ for sharp and $A_{i} \cap B_{i}=\varnothing$ for crosslink), each position $i$ for which this relation is satisfied will be called a specific position of a cube.

- The operation executed on the literals of position $i$ (like $\neg B_{i} \cap A_{i}$ for sharp and $A_{i} \cup B_{i}$ for crosslink).

- The operation executed on literals before the position i (like copying the literals from $A_{i+1}$ to $A_{N}$ to the resultant cube in both the above examples).

- The operation executed on literals after the position i (like copying the literals from $A_{1}$ to $A_{i-1}$ for sharp, and from $B_{1}$ to $B_{i-1}$ for crosslink, to the respective positions from 1 to $\mathrm{i}-1$ of the resultant cube. 


\section{SIMPLE COMBINATIONAL OPERATIONS}

Combinational cube calculus operations are defined as bit-wise functions on the bits of the arguments. There is no need for defining active, before and after positions for combinational cube calculus operations. For consistency of the description, however, one can imagine that all the literals in the case of the combinational cube calculus function are of type before. This way the same computational mechanism can be used to calculate both sequential and combinational cube calculus functions. Simple combinational operations are defined as follows:

$$
A o p_{s c} B=\left\{C_{k} \mid C_{k}=X_{1}^{\operatorname{bef}\left(A_{1}, B_{1}\right)}, \ldots, X_{N}^{\operatorname{bef}\left(A_{N}, B_{N}\right)}\right\}
$$

Simple combinational operations include intersection and supercube, and produce a single resultant cube for each pair of operand cubes.

\section{$\underline{\text { Intersection }}$}

The definition of the intersection operation for cubes A and B in positional notation is:

$$
A \cap B=\left\{\begin{array}{cc}
{\left[A_{1} \cap B_{1}, \ldots, A_{N} \cap B_{N}\right]} & \text { if there is no such } i \text { that } A_{i} \cap B_{i}=\varnothing \\
\phi & \text { otherwise }
\end{array}\right.
$$

where: $A_{i}$ is the $i$-th literal (position) of cube $A, A_{i} \cap B_{i}$ is a set intersection of sets $A_{i}$ and $\mathrm{B}_{\mathrm{i}}$ in positional notation; $\varnothing$ denotes a vector of zeros: $00 \ldots 0$ with as many bits as variable $i$ has values; $\phi$ is an empty set (a contradiction which may be signalized). The resultant cube $\mathrm{C}$, is the cube that is included in both $\mathrm{A}$ and $\mathrm{B}$. Figure 14 shows an example of two input cubes $\mathrm{A}$ and $\mathrm{B}$, and their overlapping of literals as seen from a Karnaugh map representation. The intersection operation is useful in finding similar literals between two 
input cubes.

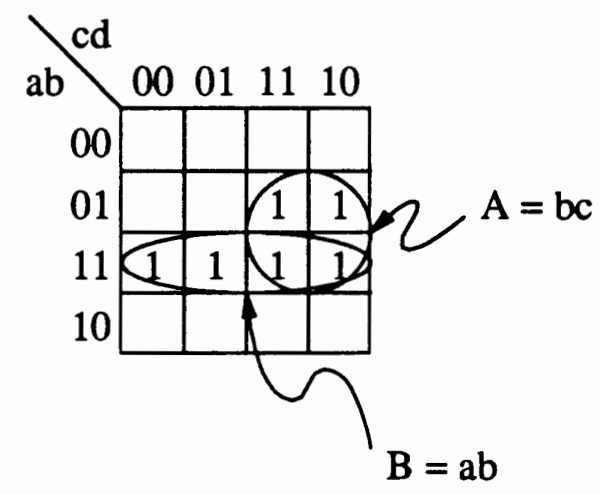

Figure 14. Input cubes A and B to be intersected.

The resultant cube produced by the intersection operation upon the input cubes $\mathrm{A}$ and $\mathrm{B}$ is the cube $\mathrm{C}$, which is the cube that is included in both A and B. The Karnaugh map of this operation may be seen in Figure 15.

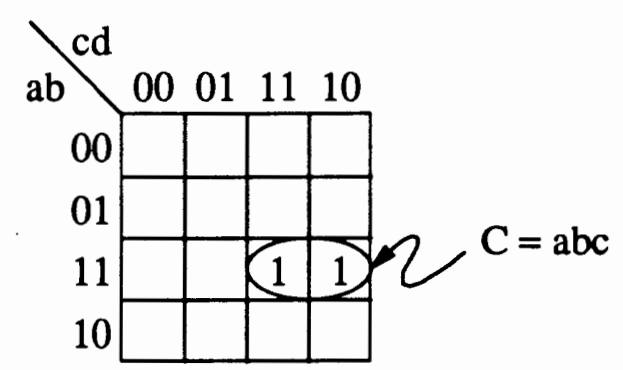

Figure 15. Resultant cube $\mathrm{C}=\mathrm{A} \cap \mathrm{B}$.

\section{Supercube}

The supercube of two cubes $\mathrm{A}$ and $\mathrm{B}$, is the smallest cube that includes all the literals of both A and B. The supercube operation of cubes A and B is defined as follows:

$$
A \cup B=\left[A_{1} \cup B_{1}, \ldots, A_{n} \cup B_{N}\right]
$$

where $A_{i} \cup B_{i}$ is a set union. Two cubes $A$ and $B$ are included in some prime implicant if their supercube does not intersect zeros. Figure 16 shows the input cubes A and B that 
will be supercubed together. The resultant cube $C$ in Figure 17 is the smallest cube that contains both input cubes A and B. As can be seen by this example, the greater the distance between the cubes, the larger the resultant cube will be.

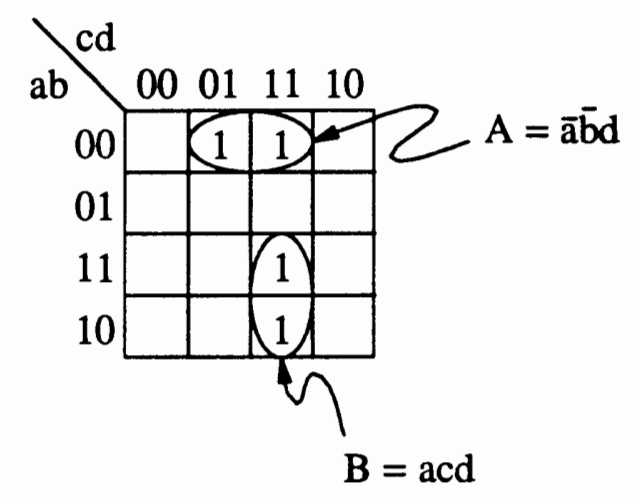

Figure 16. Input cubes $A$ and $B$ to be supercubed.

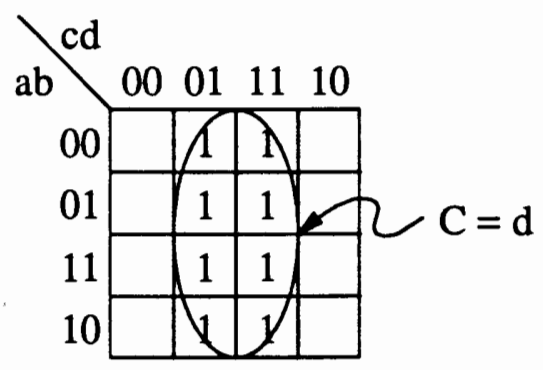

Figure 17. Resultant cube $C=A \cup B$.

\section{COMPLEX COMBINATIONAL OPERATIONS}

There is one exception to the presented scheme. The operation called "prime" is calculated as if all the specific positions were active at the same time. There is only one output cube for this operation, that can be described as:

$$
\begin{gathered}
C=A^{\prime} B=X_{1}^{\operatorname{bef}\left(A_{1}, B_{1}\right)} \cdots X_{k-1}^{\operatorname{bef}\left(A_{k-1}, B_{k-1}\right)} X_{k}^{\operatorname{act}\left(A_{k}, B_{k}\right)} X_{k+1}^{\operatorname{bef}\left(A_{k+1}, B_{k+1}\right)} \cdots \\
\left.X_{1-1}^{\operatorname{bef}\left(A_{l-1}, B_{1-1}\right)} X_{1}^{\text {act }\left(A_{1}, B_{1}\right)} X_{1+1}^{\operatorname{bef}\left(A_{l+1}, B_{k+1}\right)} \cdots X_{N}^{\operatorname{bef}\left(A_{N}, B_{N}\right)}\right\}
\end{gathered}
$$


where literals with indices " $\mathrm{k}$ " and "l" are in specific (in this case the same as active) positions. The number of the specific positions is arbitrary, the specific positions obtained are when relation "rel" is satisfied. The values of other literals are calculated according to the "bef" function. Function "aft" is not used.

From the above considerations it follows, that each of the "bef", "aft" and "act" functions is actually a function of two single bits. Therefore each of them can be described completely by a 2-variable Karnaugh map. Since a 2-variable Karnaugh map has four true minterms enumerated $0,1,2$, and 3 , it can be completely specified by the string of four bits corresponding to those minterms. For instance $(0,1,1,1)$ will correspond to minterms of numbers 1,2 , and 3 , which is $\bar{A} \cdot B, A \cdot \bar{B}$, and $A \cdot B$.

To specify completely the "rel" function one more bit is needed, which serves to determine the method of combining partial relations from single bits (the OR-type relation or the AND-type relation). AND-type means that all ITs must be satisfied within a variable to satisfy the relation. OR-type means that at least one IT must be satisfied within a variable to satisfy the relation.

This way a whole class of sequential CC operations can be described by specifying $4+4+4+1=13$ bits. One more bit can be used to denote that "prime" function is going to be calculated, and one bit to distinguish between sequential and combinational operations. In practical implementation a carry signal is used to help combine partial relations for particular bits. Figure 18 shows the input cubes $A$ and $B$ upon which the prime operation will be executed. By paying careful attention to Equation 11, we can see how the resultant cube is produced as presented here in Figure 19. 


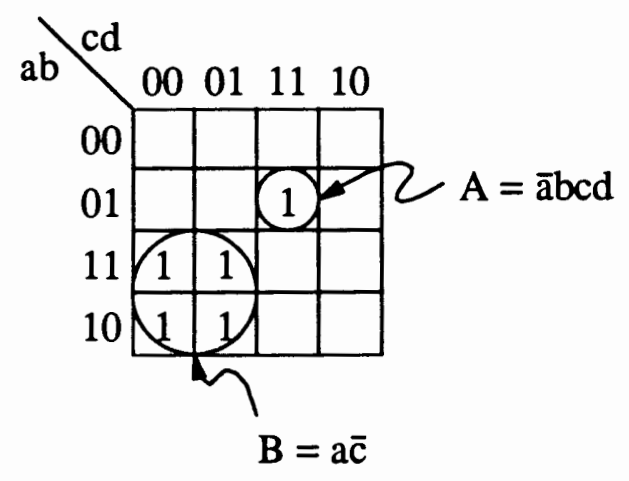

Figure 18. $A^{\prime} B=\bar{a} b c d$ ' $a \bar{c}$.

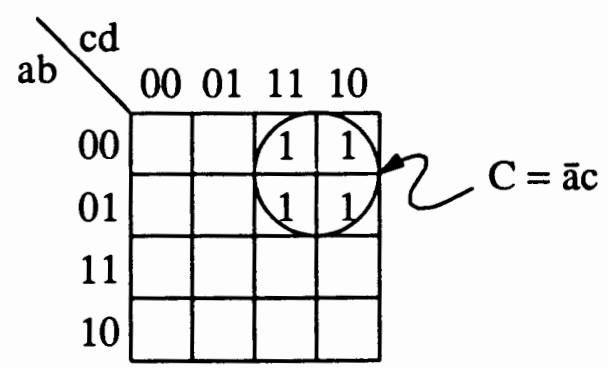

Figure 19. Resultant cube C = A ' B = āc.

This chapter showed the cube calculus operations that are currently implemented in the CCM2. Future generations of the machine will have a larger group of calculations available and will also be able to operate upon symbols. 


\section{CHAPTER IV}

\section{ITERATIVE LOGIC UNIT}

The architecture of the Cube Calculus Machine II (CCM2) results from an attempt to optimize the execution of sequential operations [13][30][42]. The architecture consists of an iterative logic array unit (ILU) with iterative signals running from left to right and from right to left of the iterative circuit of Positional State Machines (PSMs). The advantage of this approach is that only resultant cubes without contradictions are generated.

The ILU recognizes the next specific position of the input cube and generates a resultant cube in each cycle. It realizes, using internal distributed control, the lowest level iterative loop. Therefore, the ILU does not need the control unit (CU) to execute the basic cube operations. This is a general concept in computer architecture consisting of taking the control of the lowest level loop off the control unit and putting it directly to the data path in a distributed form. Figure 20 shows this approach of using an array of positional state machines (PSMs) which communicate among themselves, each with its left and right neighbors. The $\mathrm{CU}$ is responsible for sending global controlling signals and initiating instructions executed by the array of FSMs. The CU receives both the predicates as well as the termination signals from the the string of FSMs. Two of these signals, the global REQUEST and the iterative NEXT signals work in an interlock

mechanism that substitutes the clock of synchronous machines with a two-phase nonoverlapping rippling waveform of iterative PSMs. 


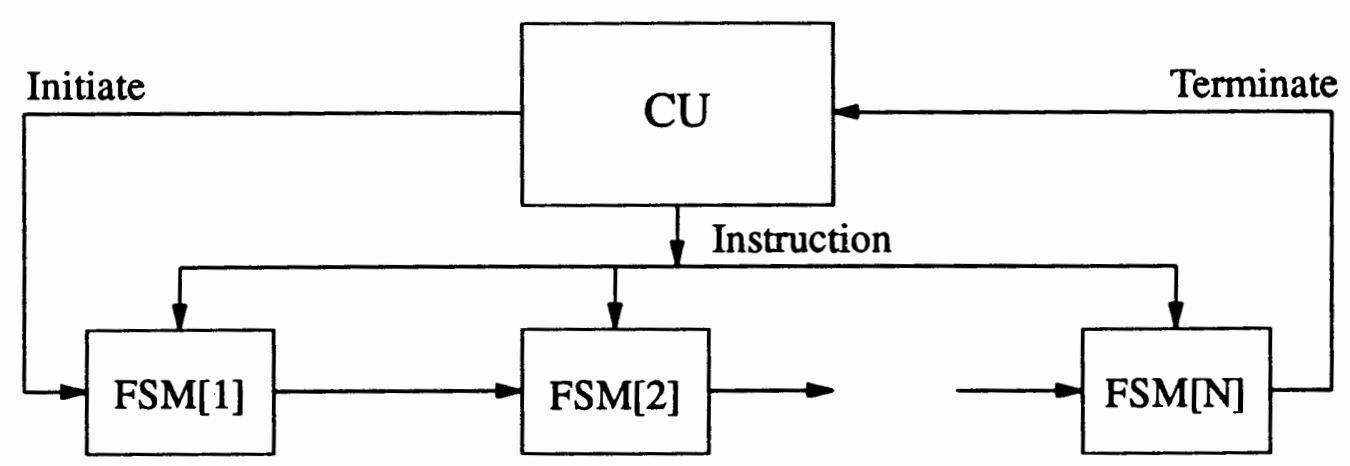

Figure 20. Cube Calculus Machine architectural model.

To help understand the advantages of this architecture, imagine each literal as a domino tile. The linear iterative array has all the dominoes lined up in a way that if the first one falls, the next will fall in sequence. For the specific positions the correspondent domino tile is removed. This way, when the control unit initiates the first domino to fall, the tiles will fall in a "domino effect" until they reach the gap left by the specific position and the domino effect will stop. At that point, the literal is processed and an output cube is generated with each of its literals being a function of its position in the array. If a domino has already fallen down it corresponds to the state "AFTER". If the literal is being processed, it corresponds to state "ACTIVE", and when a domino is still standing, it corresponds to state "BEFORE". The control unit begins the cycle again by pushing the first remaining domino until there are no more dominoes left standing.

This is of course only a general analogy, since dominoes fall one after another, and in our design a group of dominoes is prepared to fall. For the CCM2, the iterative circuit has a ring configuration with the control unit serving as the first and last domino in the ring. This way, it is simple for the control unit to observe the fact that all literals have been processed, without the need to keep track of which literal is being processed and how many remain to be processed. 
A processor that processes a literal at a time would push a single domino for each cycle and try to improve performance by increasing the rate at which it processes each literal. Among the disadvantages of such a method is that it generates cubes with contradictions that have to be removed. If a circuit to recognize and remove cubes with contradictions were integrated into the architecture, then the rate of generation of resultant cubes would be irregular. The rate of generation of resultant cubes in the CCM2 architecture is regular, making it suitable for pipelining and systolic processing, which allows the construction of large parallel structures.

This chapter describes the ILU in detail, breaking each Iterative Cell (IT) down into easily understood subsections with their respective gate-level logic realization. This section starts with descriptions of the Instruction Register (IR) and the Control Unit which provide inputs and control to the ILU.

\section{INSTRUCTION REGISTER}

The Instruction Register (IR) contains the registers from which each IT obtains the necessary data for operation. These registers contain information concerning the mode in which the CCM2 is running, the current operation to be executed, the relations and prerelations necessary in the operation and the definition of specific positions in each cube. The data concerns both the ILU and the CU. The seven registers needed are listed as follows:

IR[0] Sequential or combinational cube calculus operation.

$$
\begin{aligned}
& \operatorname{IR}[0]=0 \text { for sequential operation. } \\
& \operatorname{IR}[0]=1 \text { for combinational operation. }
\end{aligned}
$$

IR[1] Chain or standalone mode. 
IR $[1]=0$ for standalone mode.

$\operatorname{IR}[1]=1$ for chain mode.

IR[2] Counter mode.

$\operatorname{IR}[2]=0$ for counter mode CNT0 which means counting in all ITs.

$\operatorname{IR}[2]=1$ for counter mode CNT1 which means counting all the variables that are satisfied.

IR[3] AND/OR type relation for the sequential part of a Cube Calculus operation (RELATION).

$\operatorname{IR}[3]=0$ for OR-type relation for RELATION.

$\operatorname{IR}[3]=1$ for AND_type relation for RELATION.

IR[4] AND/OR type relation for the condition (the combinational part PRERELATION) of a sequential cube calculus operation.

$\operatorname{IR}[4]=0$ for OR-type relation for PRERELATION.

IR[4] = 1 for AND_type relation for PRERELATION.

IR[5,6] Determines the type of sequential algorithm. TABLE I shows the values needed at IR5 and IR6 to match the desired sequential operation. 
TABLE I

\section{DEFINITION OF SEQUENTIAL OPERATION}

\begin{tabular}{|c|c|c|}
\hline $\operatorname{IR}[5]$ & IR[6] & sequential operation \\
\hline \hline 0 & 0 & only main loop \\
\hline 0 & 1 & sharp \\
\hline 1 & 0 & consensus \\
\hline 1 & 1 & crosslink \\
\hline
\end{tabular}

The IR has been realized on the CCM2 board as a bank of eight dip-switches that can be user configured. This approach was taken since there is no interface to a host computer at this time. 


\section{CONTROL UNIT}

The task of the Control Unit (CU) of the CCM2 is to generate signals for sequential cube calculus operations and for operating the CCM2 in pipeline mode. In this mode there is a pipe of CCM2-processors and some of the input cubes can have contradictions. In a such case, operations should be not executed on them. In pipeline mode, the CU provides the ILU with signals to control if one of the input cubes is a contradictory one. Since the pipeline mode will not be explored in this thesis, the CU is needed only for providing the ILU the global signals necessary for executing sequential operations. To perform sequential cube calculus operations, the $\mathrm{CU}$ has to provide the ILU with the correct control signals to calculate the solution cubes.

The CU implemented in this design provides minimal control to the ILU. Since there will be no interfacing to a data bus, the only control needed is for the determination of the prerelation and relation that is needed for the sequential operation. The CU must also communicate with the ILU to determine when the next specific position must be calculated. This is done by clocking the Positional State Machine (PSM) of each IT with the REQUEST signal generated by the CU. Figure 21 shows the state diagram of the CU.

The operation is as follows: At State 0 , the CU determines whether the CCM2 is functioning in standalone or pipeline mode, and whether the cube calculus operation will be sequential or combinational. If $\operatorname{IR} 0=1$, a combinational operation will be executed and the $\mathrm{CU}$ is not needed since only one resultant cube will be produced. If $\mathbb{I R} 0=0$, a sequential operation will be executed and the CU will progress to State 1 if IR1=0. IR1 determines whether the CCM2 will function in a standalone or pipeline mode. Since the goal is to verify the proper operation of the ILU and the pipelined mode is not implemented, we will always operate in standalone mode $(\mathbb{R} 1=0)$. Once IR0 and IR1 both equal zero, the CU will issue the signals CLEAR and REQUEST to the ILU. The 
signal CLEAR is sent to each IT of the ILU to reset the PSMs to the state BEFORE. The signal REQUEST is also sent to each IT to clock the PSMs, aiding in the reseting of the ILU.

At State 1, IR0 is checked again to see if the operation will truly be sequential. If in fact it is a sequential operation, IR4, IR5 and IR6 are checked to determine if the operation selected will need to check a prerelation before the relation may be determined. IR5 and IR6 define the operation being executed that needs to be checked for a prerelation. If IR5 and IR6 both equal zero, a prerelation check is not needed for the specified operation. For this model, we will assume all operations will go through this check. If IR4=1, the prerelation is AND-type, otherwise it is an OR-type prerelation. Once the prerelation has been determined, the CU progresses to State 2 and outputs the AND_OR signal to the ILU.

At State 2, the relation of the operation is finally determined. IR3 determines whether the relation needed to calculate the specific positions of the input cubes is ANDtype or OR-type by the value 1 or 0 respectively. On its progression to State 3 , the CU outputs the AND_OR, REQUEST and NEXT signals to the ILU. The NEXT signal activates the interlock mechanism of the PSMs in the ILU, while the REQUEST signal clocks the PSMs from the BEFORE to the ACTIVE state. The CU then ripples on to State 4 while it still sends the NEXT signal to the ILU.

At State 4, the CU will remain at this state until the READY signal is received from the ILU. This signal tells the control unit that a resultant cube has been generated. Once the READY signal has been received, the CU transits to State 5 which is merely a delay state. At State 6 if the CU receives the NEXT signal from the ILU declaring that there are no more resultant cubes to be generated, it transits on to State 0 , waiting to perform the next operation. If the NEXT signal is not received, this indicates that there are 
additional resultant cubes that need to be calculated. The control unit transits back to State 4 and produces the REQUEST signal, thus clocking the PSMs to their next state in order to produce the next resultant cube. The CU transits through continually, until is finally receives the NEXT signal from the ILU, and completes its operation. 


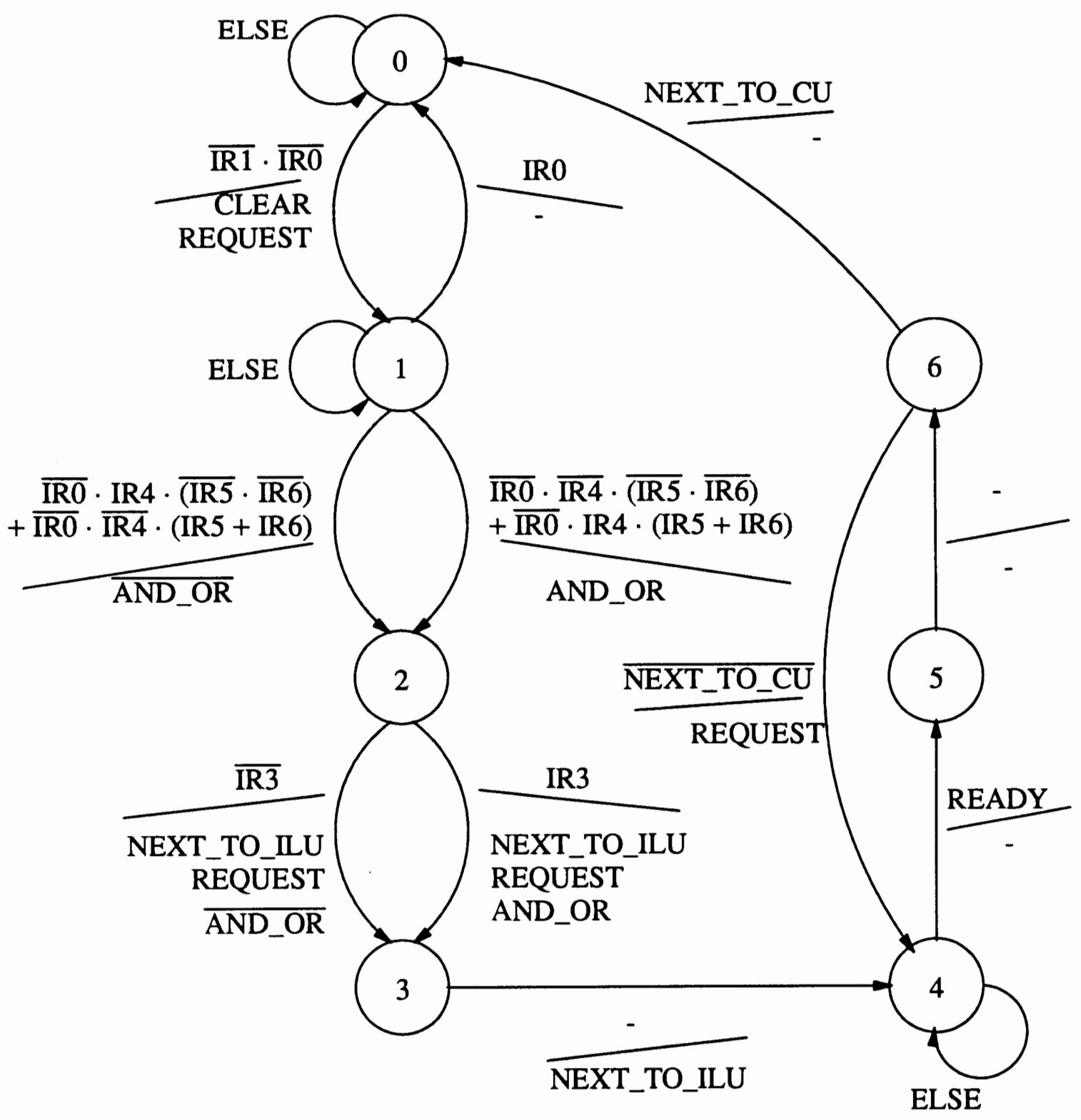

Figure 21. Control unit of the CCM2. 


\section{ITERATIVE CELL}

Each basic block or cell within the ILU is referred to as an iterative cell (IT). The IT cells are enumerated from left to right: IT[1], IT[2], ... IT[n]. The number of ITs is denoted by $n$, so that the number of bits is $2 n$, allowing the processing of $n$ binary variables, or 2 bits of a multi-valued variable. Each IT contains combinational logic and a positional state machine (PSM), influencing the interpretation of the microinstructions. Thus, each IT acts as a small processing unit that processes a portion of the input cube in parallel with the other processors that are connected in the linear array.

Local signals within each IT have the index of $i$. Horizontal signals between ITs flowing to the right such as CARRY $\mathrm{C}_{\mathrm{i}}$ and $\mathrm{NEXT}_{\mathrm{i}}$, correspond to an index of $\mathrm{i}+1$. Horizontal signals between ITs flowing to the left such as $\mathrm{CONF}_{\mathrm{i}}$, correspond to an index of $i-1$. Vertical signals such as REQUEST $T_{i}, \operatorname{CLEAR}_{i}, \operatorname{READY}_{i}, C_{i 0}$ and $C_{i 1}$ are global signals respectively received by and sent to the $C U$. Vertical signals such as $A_{i 0}, B_{i 0}, A_{i 1}$, $B_{i 1}$, RIGHT_E $E_{i}$ and $W_{i}$ are register stored values of the input cubes and necessary information about the operations to be executed upon these variables. Figure 22 shows the block diagram of a standard IT cell and the respective signals and their indexing.

As previously stated, the $\mathrm{CU}$ acts as the initiator and terminator of the operations upon a cube, realized as IT[0] and IT[17] respectively in the chain of ITs. Figure 23 shows the structure of the ILU with the incoming and outgoing signals from the cells being initiated and terminated by the $\mathrm{CU}$. 


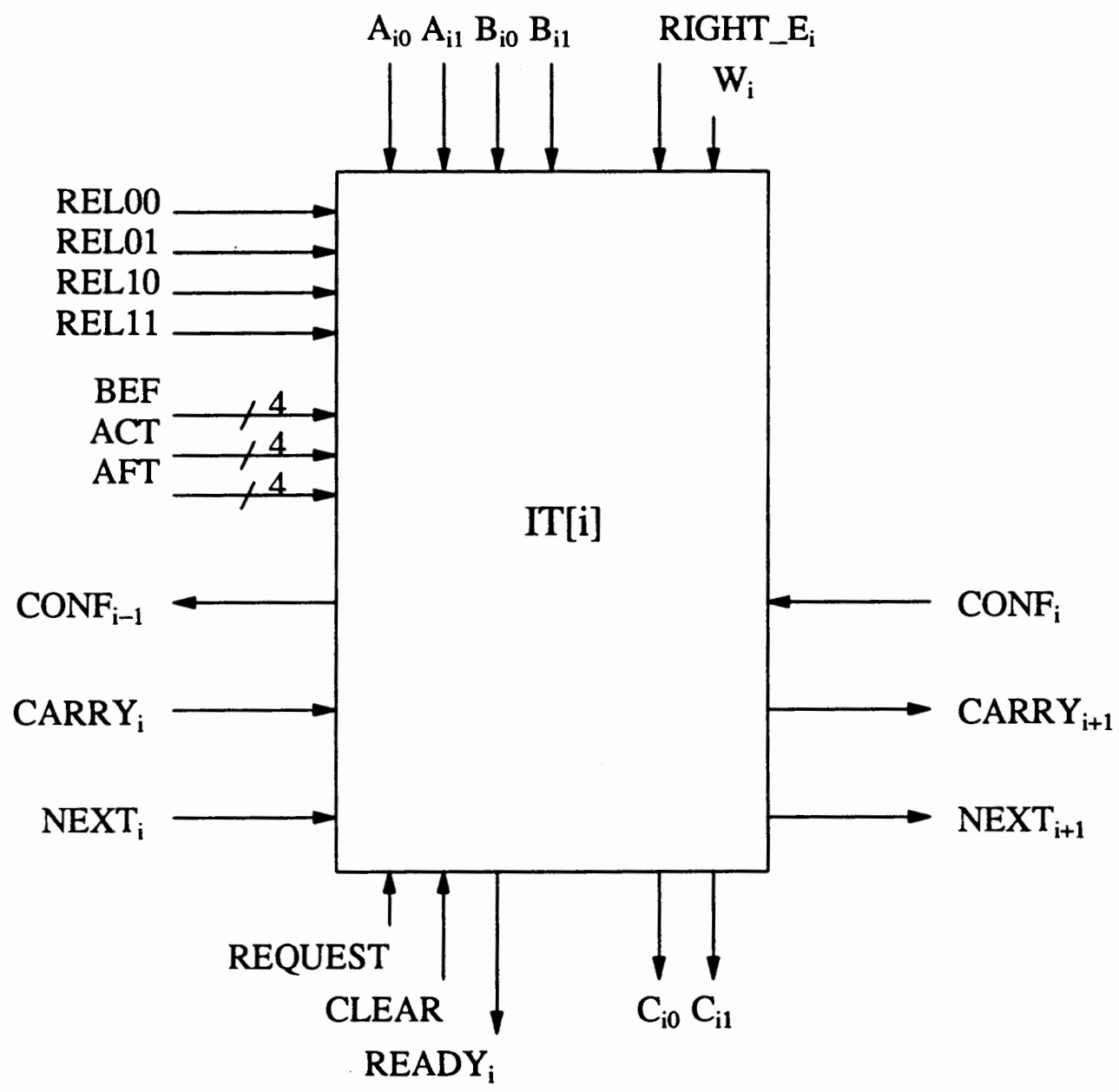

Figure 22. Iterative cell (IT). 


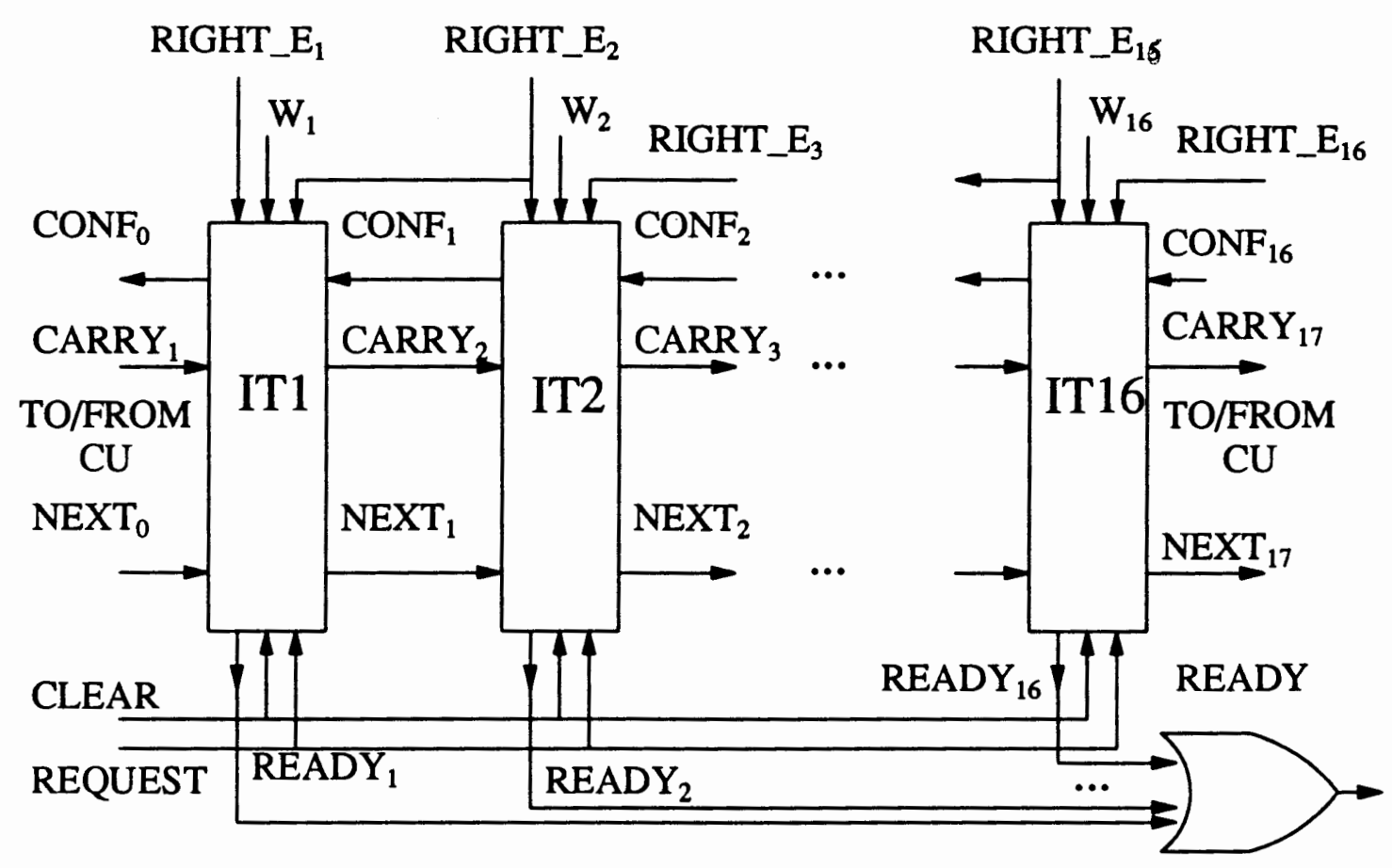

Figure 23. The iterative logic unit (ILU).

For explanation purposes, each IT is broken down into four parts to aid in the understanding of its structure. These portions are Relation, Variable Generate, State and Count. They are fully described in the following sections in their respective order.

\section{$\underline{\text { Relation }}$}

From the considerations stated in Chapter III, it follows that each of the BEFORE, AFTER and ACTIVE functions can be composed from the same boolean functions of two single bits. Therefore each bit can be described completely by a 2-variable Karnaugh map. Since a 2-variable Karnaugh map has four true minterms enumerated 0, 1, 2, and 3, it can be completely specified by the string of four bits corresponding to those minterms. For instance $(0,1,1,1)$ will correspond to minterms of numbers 1,2 , and 3 , which is $\bar{a} \cdot b$, $a \cdot \bar{b}$, and $a \cdot b$. This can be seen in the Karnaugh map in Figure 24. 
To calculate one of the functions BEFORE, AFTER and ACTIVE in the CCM2, one IT can be used to calculate one pair of bits $\mathrm{CO}_{\mathrm{i}}$ and $\mathrm{Cl}_{\mathrm{i}}$ of the resultant cube in positional notation. This portion of the IT can be regarded as two 4-to-1 multiplexors, with the two bits $\mathrm{AO}_{\mathrm{i}}$ and $\mathrm{B} \mathrm{O}_{\mathrm{i}}$ as the address (switching) inputs of the first multiplexor, and bits $\mathrm{A} 1_{\mathrm{i}}$ and $B 1_{i}$ as the data (switched) inputs of the second multiplexor. To calculate bit $\mathrm{i}$ of function REL, with $\operatorname{REL}_{i}=\overline{A_{i}} \cdot B_{i}+A_{i} \cdot \overline{B_{i}}+A_{i} \cdot B_{i}=$ [0111], the bits $0,1,1$, and 1 are sent to the data inputs of the multiplexor. The values of the address inputs $A 0_{i}$ and $B 0_{i}$ will select the valid minterm from the Karnaugh map, and the correct function value, $\mathrm{CO}_{\mathrm{i}}$, will be sent to the output. Let us observe that in the CCM2 architecture each IT calculates two bits of the resulting cube $C$. These can denote a binary variable or 2 values of a multi-valued variable in positional notation. Figure 25 shows the multiplexor realization for the REL signals.

The function of two bits, of which two are calculated by one IT, is completely specified by the four bits that are sent to the data inputs of the multiplexor. For each of the functions BEFORE, AFTER and ACTIVE of each cube calculus operation, another set of four bits will be sent to the inputs. These sets of four bits are equal for all IT's within a literal. All sets of four bits are stored in registers in the CCM2, which means that the set of possible $\mathrm{CC}$ operations can be easily changed.

To specify completely the REL function one more bit is needed, which serves to determine the method of combining partial relations from single bits (the OR-type relation or the AND-type relation). Thus we need $4+1$ bits to specify the relation.

AND-type means that all ITs must be satisfied within a variable to satisfy the relation. OR-type means that at least one IT must be satisfied within a variable to satisfy the relation. In the case of the crosslink operation, the function REL is: $A_{j} \cup B_{j}=\varnothing$, or $a_{i} \cdot b_{i}=0$, or (from De Morgan's theorem) $\overline{a_{i}}+\overline{b_{i}}=1$, for every $i \in 1 \ldots N$. Therefore, 
the REL function of the crosslink operation is as follows:

$$
\operatorname{REL}\left(A_{j}, B_{j}\right)=\left(\overline{a_{1}}+\overline{b_{1}}\right) \cdot\left(\overline{a_{2}}+\overline{b_{2}}\right) \cdot, \ldots, \cdot\left(\overline{a_{N}}+\overline{b_{N}}\right)
$$

Where $A_{j}=\left[a_{1}, \ldots, a_{N}\right]$ and $B_{j}=\left[b_{i}, \ldots, b_{N}\right]$ are the value-sets of the two literals of the argument cubes $\mathrm{A}$ and $\mathrm{B}$.

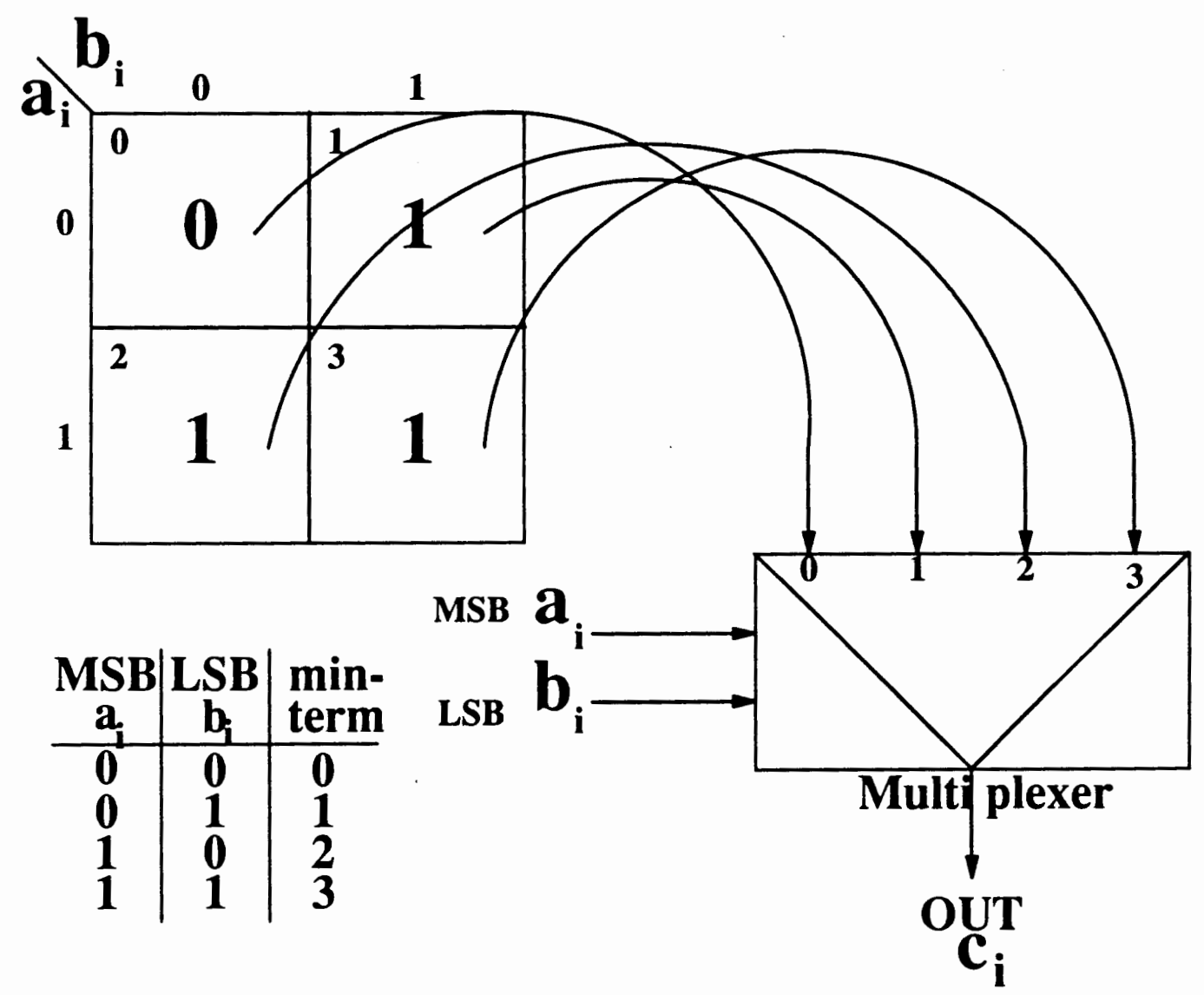

Figure 24. Karnaugh realization of Relation.

The crosslink is an "AND-type" function. An example of an "OR-type" function is the sharp, where REL is: $\neg\left(B_{j} \supseteq A_{j}\right)$, or $a_{i} \cdot \neg b_{i}=0$ for every $i \in 1, \ldots, N$. TABLE II shows how the function $a_{i} \cdot \neg b_{i}=0$ was found from REL $=\neg\left(B_{j} \supseteq A_{j}\right)$. 
TABLE II

THE FUNCTION REL

\begin{tabular}{|cc||c|c|c|}
\hline $\mathrm{a}_{\mathrm{i}}$ & $\mathrm{b}_{\mathrm{i}}$ & $\mathrm{B}_{\mathrm{j}} \supseteq \mathrm{A}_{\mathrm{j}}$ & $\neg\left(\mathrm{B}_{\mathrm{j}} \supseteq \mathrm{A}_{\mathrm{j}}\right)$ & $\mathrm{a}_{\mathrm{i}} \cdot \neg \mathrm{b}_{\mathrm{i}}$ \\
\hline \hline 0 & 0 & 1 & 0 & 0 \\
0 & 1 & 1 & 0 & 0 \\
1 & 0 & 0 & 1 & 1 \\
1 & 1 & 1 & 0 & 0 \\
\hline
\end{tabular}

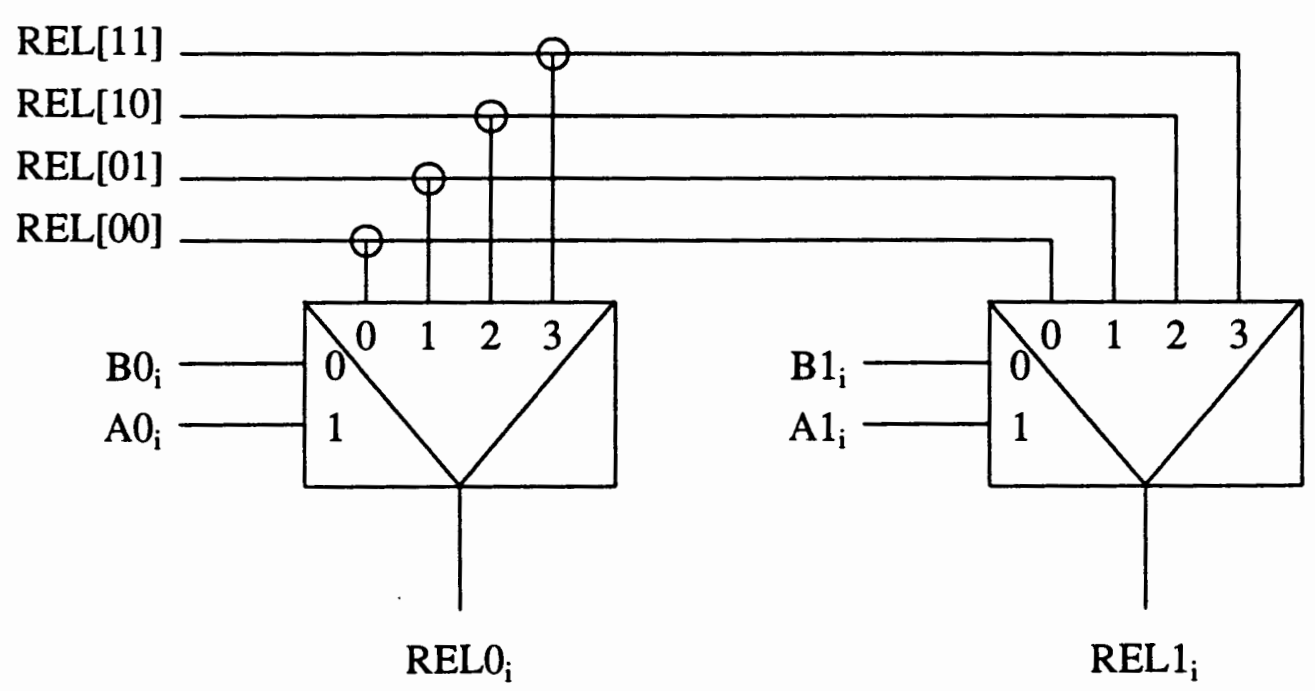

Figure 25. Relation multiplexing.

The first column shows two bits $a_{i}$ and $b_{i}$, from the value sets $A_{j}$ and $B_{j}$ of the argument cubes $\mathrm{A}$ and $\mathrm{B}$. The next two columns show the value of the negated function REL and the function REL itself. In the third column we can see that REL, as function of $a_{i}$ and $b_{i}$, is $a_{i} \cdot \neg b_{i}$. Therefore, the REL function of the sharp operation is as follows:

$$
\operatorname{REL}\left(A_{j}, B_{j}\right)=\left(a_{1} \cdot \neg b_{1}\right)+\left(a_{2} \cdot \neg b_{2}\right)+, \ldots,+\left(a_{N} \cdot \neg b_{N}\right)
$$


We see that a whole class of sequential CC operations can be described by specifying 4 bits for each function BEFORE, AFTER, ACTIVE, REL, and 1 bit for the type of relation (AND / OR), which results in a total of $4+4+4+4+1=17$ bits. One more bit can be used to denote that the "prime" function is going to be calculated, and one bit to distinguish between sequential and combinational operations. Figure 26 shows the realization of this portion of the calculation of resultant cubes. 


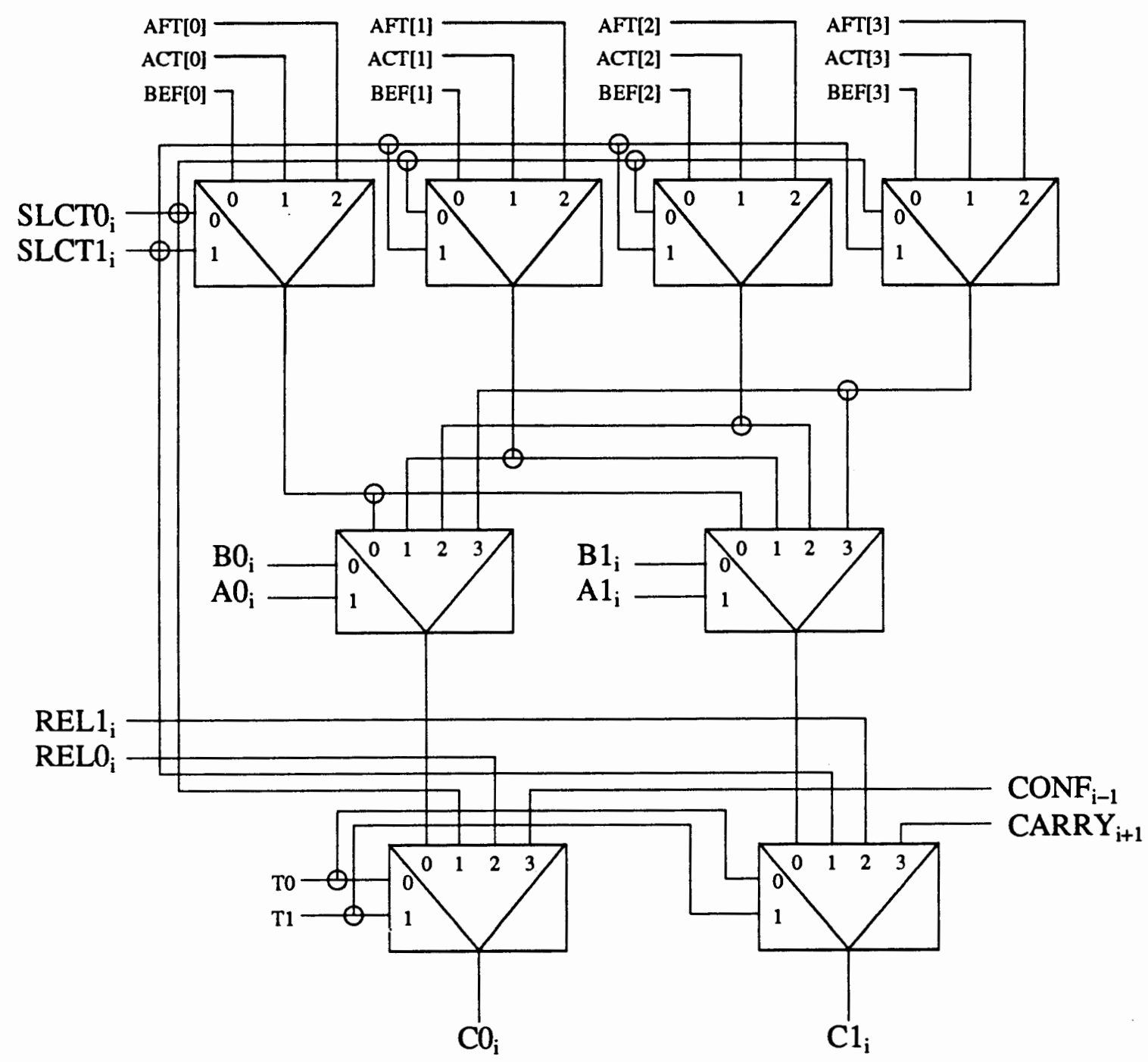

Figure 26. Resultant cube multiplexing.

In practical implementation a CARRY signal is used to help combine partial relations for particular bits. (It can be shown that for relations like $A=B, A \supseteq B$ carry signal of one bit is enough, while for the relations like $\mathrm{A} \leq \mathrm{B}, \mathrm{A}<\mathrm{B}$ or $\mathrm{A} \supset \mathrm{B}$ two-bit carry signal is required. For example, to calculate the relation $A=B$, each IT[i] indicates to the next IT, IT $[i+1]$, that either all bits $\left[a_{j}=b_{j}\right]$ for $j=1, \ldots, i$, or that there is such 
$\mathrm{j} \leq \mathrm{i}$ that $\mathrm{a}_{\mathrm{j}} \neq \mathrm{b}_{\mathrm{j}}$. There are only two possible situations, which can be indicated by a single carry-bit. To calculate the relation $\mathrm{A} \supset \mathrm{B}$, more possible situations have to be indicated. For the relation to be true, there must be a j such that $a_{j} \supset b_{j}$, and there can not be a $j$ such that $a_{j} \subset b_{j}$. If $a_{j}=b_{j}$ for $j=1, \ldots, i$, the relation can be either true or false for the complete literals A and B. Two carry-bits are needed to indicate this to the next IT. The CCM2 does not implement this second carry-bit yet, but the CCM 2.5 and the CCM3, have this generalized carry, even in a much more generalized form. The basic sequential cube calculus operations are shown in TABLE III.

TABLE III

SEQUENTIAL CUBE CALCULUS OPERATIONS

\begin{tabular}{|l|c||c|c||c|c|c|}
\hline \multicolumn{1}{|c|}{ function } & \multicolumn{1}{|c||}{ notation } & \multicolumn{3}{c||}{ relation (REL) } & \multicolumn{3}{c|}{ output operation } \\
\hline & & REL & $\begin{array}{c}\text { AND } \\
\text { OR }\end{array}$ & $\begin{array}{c}\text { BEFORE } \\
\text { (BEF) }\end{array}$ & $\begin{array}{c}\text { ACTIVE } \\
\text { (ACT) }\end{array}$ & $\begin{array}{c}\text { AFTER } \\
\text { (AFT) }\end{array}$ \\
\hline crosslink & A $\mathrm{AB}$ & $\mathrm{A}_{\mathrm{i}} \cap \mathrm{B}_{\mathrm{i}}=\varnothing$ & AND & $\mathrm{A}_{\mathrm{i}}$ & $\mathrm{A}_{\mathrm{i}} \cup \mathrm{B}_{\mathrm{i}}$ & $\mathrm{B}_{\mathrm{i}}$ \\
sharp & $\mathrm{A} \# \mathrm{~B}$ & $\neg\left(\mathrm{B}_{\mathrm{i}} \supseteq \mathrm{A}_{\mathrm{i}}\right)$ & OR & $\mathrm{A}_{\mathrm{i}}$ & $\neg \mathrm{B}_{\mathrm{i}} \cap \mathrm{A}_{\mathrm{i}}$ & $\mathrm{A}_{\mathrm{i}}$ \\
disjoint sharp & $\mathrm{A} \# \mathrm{~B} \mathrm{~B}$ & $\neg\left(\mathrm{B}_{\mathrm{i}} \supseteq \mathrm{A}_{\mathrm{i}}\right)$ & OR & $\mathrm{A}_{\mathrm{i}}$ & $\neg \mathrm{B}_{\mathrm{i}} \cap \mathrm{A}_{\mathrm{i}}$ & $\mathrm{A}_{\mathrm{i}} \cap \mathrm{B}_{\mathrm{i}}$ \\
standard cons & $\mathrm{A} * \mathrm{~B}$ & 1 & AND & $\mathrm{A}_{\mathrm{i}} \cap \mathrm{B}_{\mathrm{i}}$ & $\mathrm{A}_{\mathrm{i}} \cup \mathrm{B}_{\mathrm{i}}$ & $\mathrm{A}_{\mathrm{i}} \cap \mathrm{B}_{\mathrm{i}}$ \\
sym cons & $\mathrm{A} * \mathrm{~s} \mathrm{~B}$ & 1 & AND & $\mathrm{A}_{\mathrm{i}} \cap \mathrm{B}_{\mathrm{i}}$ & $\mathrm{A}_{\mathrm{i}} \cup \mathrm{B}_{\mathrm{i}}$ & $\mathrm{A}_{\mathrm{i}} \cap \mathrm{B}_{\mathrm{i}}$ \\
asym cons & $\mathrm{A} * \mathrm{a} \mathrm{B}$ & $\neg\left(\mathrm{B}_{\mathrm{i}} \supseteq \mathrm{A}_{\mathrm{i}}\right)$ & OR & $\mathrm{A}_{\mathrm{i}} \cap \mathrm{B}_{\mathrm{i}}$ & $\mathrm{A}_{\mathrm{i}} \cup \mathrm{B}_{\mathrm{i}}$ & $\mathrm{A}_{\mathrm{i}} \cap \mathrm{B}_{\mathrm{i}}$ \\
complement & $\neg \mathrm{A}$ & $\mathrm{A}_{\mathrm{i}} \neq 1$ & OR & 1 & $\neg \mathrm{A}_{\mathrm{i}}$ & 1 \\
\hline
\end{tabular}

TABLE IV shows the actual values for REL, AND_OR, BEFORE, ACTIVE, and AFTER as determined previously and shown in Figure 24. 


\section{TABLE IV}

\section{SEQUENTIAL CUBE CALCULUS OPERATIONS BIT-WISE VALUES}

\begin{tabular}{|l|c||c|c||c|c|c|}
\hline \multicolumn{1}{|c|}{ function } & \multicolumn{1}{c||}{ notation } & \multicolumn{2}{c||}{ relation (REL) } & \multicolumn{3}{c|}{ output operation } \\
\hline & & REL & $\begin{array}{c}\text { AND/ } \\
\text { OR }\end{array}$ & $\begin{array}{c}\text { BEFORE } \\
\text { (BEF) }\end{array}$ & $\begin{array}{c}\text { ACTIVE } \\
\text { (ACT) }\end{array}$ & $\begin{array}{c}\text { AFTER } \\
\text { (AFT) }\end{array}$ \\
\hline crosslink & A a B & 1110 & 1 & 0011 & 0111 & 0101 \\
sharp & A \# B & 0010 & 0 & 0011 & 0010 & 0011 \\
disjoint sharp & A \#d B & 0010 & 0 & 0011 & 0010 & 0001 \\
standard cons & A * B & 1111 & 1 & 0001 & 0111 & 0001 \\
sym cons & A * s B & 1111 & 1 & 0001 & 0111 & 0001 \\
asym cons & A * a B & 0010 & 0 & 0001 & 0111 & 0001 \\
complement & $\neg$ A & 1100 & 0 & 1111 & 1100 & 1111 \\
\hline
\end{tabular}

The crosslink has an AND-type relation, because the relation $A_{i} \cap B_{i}=\varnothing$ is only satisfied if it is satisfied in every bit. The standard and symmetric consensus operations use relation "1" which is also an AND-type relation. Since relation "1" means "always satisfied", it does not really matter what type of relation is used. Since it is global within a variable, AND-type relation is used.

The standard and symmetric consensuses seem to be equal, but the standard consensus uses a prerelation (distance $(A, B)=1$ ), and the symmetric consensus does not. The asymmetric consensus uses a different relation.

The sharp, the disjoint sharp and the asymmetric consensus use the OR-type relation

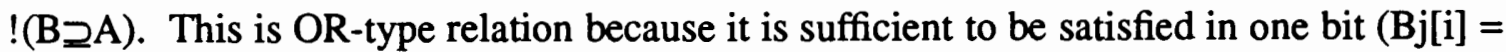
$0, A j[i]=1)$ to be satisfied in the entire variable. $B y A_{j}[i], j=0,1$, we denote two bits of signal $\mathrm{A}[\mathrm{i}]$ in IT[i], so $\mathrm{A}_{\mathrm{j}}[\mathrm{i}]=\mathrm{A}_{0}[\mathrm{i}]^{\circ} \mathrm{A}_{1}[\mathrm{i}]$, where ${ }^{\circ}$ means concatenation of signals.

TABLES $V$ and VI show the basic simple combinational and complex combinational operations. 
TABLE V

SIMPLE COMBINATIONAL CUBE CALCULUS OPERATIONS

\begin{tabular}{|l|c||c|c||c|c|c|}
\hline function & \multicolumn{1}{|c||}{ notation } & \multicolumn{2}{c||}{ relation (REL) } & \multicolumn{3}{c|}{ output operation } \\
\hline & & REL & $\begin{array}{c}\text { and/ } \\
\text { OR }\end{array}$ & $\begin{array}{c}\text { BEFORE } \\
(\mathrm{BEF})\end{array}$ & $\begin{array}{c}\text { ACTIVE } \\
\text { (ACT) }\end{array}$ & $\begin{array}{c}\text { AFTER } \\
\text { (AFT) }\end{array}$ \\
\hline \hline $\begin{array}{l}\text { intersection } \\
\text { supercube }\end{array}$ & $\mathrm{A} \cap \mathrm{B}$ & 1 & $\mathrm{AND}$ & $\mathrm{A}_{\mathrm{i}} \cap \mathrm{B}_{\mathrm{i}}$ & - & - \\
\hline
\end{tabular}

TABLE VI

COMPLEX COMBINATIONAL CUBE CALCULUS OPERATIONS

\begin{tabular}{|l|c||c|c||c|c|c|}
\hline function & \multicolumn{1}{|c||}{ notation } & \multicolumn{2}{c||}{ relation (REL) } & \multicolumn{3}{c|}{ output operation } \\
\hline & & REL & $\begin{array}{c}\text { and } \\
\text { OR }\end{array}$ & $\begin{array}{c}\text { BEFORE } \\
\text { (BEF) }\end{array}$ & $\begin{array}{c}\text { ACTIVE } \\
\text { (ACT) }\end{array}$ & $\begin{array}{c}\text { AFTER } \\
\text { (AFT) }\end{array}$ \\
\hline prime & $\mathrm{A}^{\prime} \mathrm{B}$ & $\mathrm{A}_{\mathrm{i}} \cap \mathrm{B}_{\mathrm{i}} \neq \varnothing$ & $\mathrm{AND}$ & $\mathrm{A}_{\mathrm{i}}$ & $\mathrm{A}_{\mathrm{i}} \cup \mathrm{B}_{\mathrm{i}}$ & - \\
\hline
\end{tabular}

For testing purposes, the values of the output bits $\mathrm{C}_{\mathrm{j}}[\mathrm{i}]$ of the IT can be switched between the resultant bits of the cube calculus operation and the internal signals $\mathrm{SLCTO}_{\mathrm{i}}$, $\operatorname{SLCT}_{\mathrm{i}}, \operatorname{REL}_{\mathrm{i}}, \operatorname{REL}_{\mathrm{i}}$ and NEXT $\mathrm{N}_{1+1}$ and $\mathrm{CONF}_{\mathrm{i}-1}$. This is implemented in two extra multiplexors, that have the two bits $\mathrm{T} 0$ and $\mathrm{T} 1$ on their address inputs. The IT receives these bits from the Instruction register. TABLE VII shows the values that will be received at $\mathrm{C} 0$ and $\mathrm{C} 1$ for each $\mathrm{IT}$ dependent upon the values of $\mathrm{T} 0$ and $\mathrm{T} 1$. Refer to Figure 26 to see how the values of $\mathrm{T} 0$ and $\mathrm{T} 1$ correspond to the values received at $\mathrm{C} 0$ and C1. 
TABLE VII

\section{RESULTANT CUBE VALUES \\ DEPENDENT ON TEST VALUES TO AND T1}

\begin{tabular}{|c|c|c|}
\hline T1 & TO & Resultant Value \\
\hline \hline 0 & 0 & Resultant Cube values $\mathrm{CO}_{i}$ and $\mathrm{Cl}_{i}$ \\
\hline 0 & 1 & SLCTO $_{i}$ and SLCT1 \\
\hline 1 & 0 & RELO $_{i}$ and $\mathrm{RELI}_{i}$ \\
\hline 1 & 1 & NEXT $_{i+1}$ and CONF \\
\hline
\end{tabular}

Four multiplexors are used to switch between the functions BEFORE, ACTIVE and AFTER. The bits $S L C T 0_{i}$ and $S L C T 1_{i}$ are the address inputs of these multiplexors, with $\mathrm{SLCT}_{\mathrm{i}}$ as the most significant bit. Therefore, the selected function is BEFORE when $\operatorname{SLCTO}_{i}=\operatorname{SLCT}_{i}=0$, it is ACTIVE when only SLCT0 $i=1$ and it is AFTER when only $\mathrm{SLCT}_{\mathrm{i}}=1$.

\section{Variable Generate}

The block Variable Generate is the block responsible for sending and receiving the iterative signals passed between the ITs within the ILU. This block receives the controlling signals determining the position of the IT within the iterative chain and produces the necessary signals and values to be passed to neighboring ITs. The signals vital to its operation are discussed below.

AND_OR. AND_OR is a signal received from the Instruction Register IR[3], indicating the type of relation for a sequential cube calculus operation. For OR-type operations, AND_OR=0. For AND-type operations, AND_OR=1.

RELATION $_{\mathrm{i}}$. This signal consists of the partial relations RELO $0_{i}$ and $R E L 1_{i}$ for the operation upon a single IT. For AND-type operations, both $\mathrm{RELO}_{\mathrm{i}}$ and $\mathrm{REL} 1_{\mathrm{i}}$ must equal 
1 to satisfy the relation of $I T_{i}$. For OR-type operations, either RELO or REL1 $1_{i}$ must equal 1 to satisfy the relation of $\mathbf{I T}_{\mathbf{i}}$.

RIGHT_E $E_{i}$. RIGHT_E signalizes the right edge of a variable within a cube. Detected in $\mathrm{IT}_{\mathrm{i}}$, it indicates that $\mathrm{IT}_{\mathrm{i+1}}$ is assigned to another literal. RIGHT_E $\mathrm{E}_{0}$ received from the control unit must equal 1 in order to signalize that the literal in the first $I T, I T_{1}$ is the first variable within a cube.

$\mathrm{W}_{\mathrm{i}}$. $\mathrm{W}_{\mathrm{i}}$ is a signal received from the Water Register, determining whether or not the corresponding $I T_{i}$ should be transparent to all signals running horizontally. If $W_{i}=1, I_{i}$ is transparent, and will not perform any processing of any literals. This register is needed when the number of bits required to represent all literals in the argument cubes is less than the number provided by all ITs. Thus, all the ITs will not be utilized for all operations.

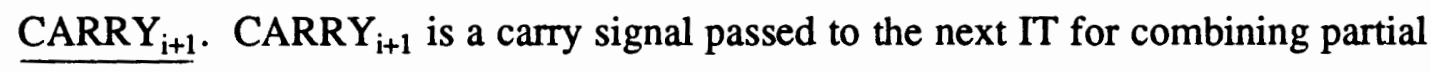
relations for literals of more than two possible values. The signal is adjusted according to the contents within the Water Register. CARRY $_{1}$ must be true at the beginning of the chain of ITs assigned to given variable, because the first meaningful CARRY $_{i}$ is generated in the first $I T_{i}$ assigned to this variable. Therefore signal RIGHT_E $E_{i}$ is present in the equation for CARRY $Y_{i+1}$. If the operation is AND_type, then

$$
\text { CARRY }_{i+1}=\left\{\begin{array}{l}
\operatorname{RELO}_{\mathrm{i}} \cdot \operatorname{REL1}_{\mathrm{i}} \text { if IT } \\
\text { CARRY is the first IT of a variable }_{\mathrm{i}} \cdot \operatorname{RELO}_{\mathrm{i}} \cdot \operatorname{REL1}_{\mathrm{i}} \text {, otherwise }
\end{array}\right.
$$

If the operation is OR_type, then

$$
\text { CARRY }_{i+1}=\left\{\begin{array}{l}
\operatorname{RELO}_{\mathrm{i}}+\operatorname{REL1}_{\mathrm{i}} \text { if } \mathrm{IT}_{\mathrm{i}} \text { is the first IT of a variable } \\
\text { CARRY }_{\mathrm{i}-1}+\operatorname{RELO} 0_{i}+\operatorname{REL1}_{\mathrm{i}} \text {, otherwise }
\end{array}\right.
$$

Taking all of the related signals into effect, the following equation results: 
CARRY $_{i+1}=$

$$
\begin{aligned}
& \text { [CARRY }_{\mathrm{i}} \cdot \mathrm{RELO}_{\mathrm{i}} \cdot \mathrm{RELl}_{\mathrm{i}} \\
& +\mathrm{RELO}_{\mathrm{i}} \cdot \mathrm{REL1}_{\mathrm{i}} \cdot \mathrm{RIGHT}^{\mathrm{R}} \mathrm{RD}_{\mathrm{i}-1} \\
& +\mathrm{CARRY}_{\mathrm{i}} \cdot \overline{\text { RIGHT_ED }_{\mathrm{i}-1}} \cdot \overline{\text { AND_OR }} \\
& +\left(\mathrm{RELO}_{\mathrm{i}}+\mathrm{REL}_{\mathrm{i}}\right) \cdot \overline{\text { AND_OR }} \\
& \left.+\mathrm{CARRY}_{\mathrm{i}}\right] \cdot \mathrm{W}_{\mathrm{i}}
\end{aligned}
$$

The CARRY $Y_{i}$ signal is created in the CCM2 with the logic circuit shown in Figure 27.

$\mathrm{CONF}_{\mathrm{i}-1} . \mathrm{CONF}_{\mathrm{i}-1}$ is the confirmation signal accounting for the Water register contents; on right edge (that is, in the last IT assigned to given variable) incoming CONF; must not be taken into account. When RIGHT_E $E_{i}=1$ then take $C_{\text {ONF }}$, otherwise take the previous $\mathrm{CONF}_{\mathrm{i}}$ from the right.

$$
\operatorname{CONF}[i-1]=\left\{\begin{array}{l}
\operatorname{CARRY}_{i}, \text { if } \mathrm{IT}_{i} \text { is the last } \mathrm{IT} \text { of the variable; } \\
\operatorname{CONF}_{i} \text {, otherwise. }
\end{array}\right.
$$

Taking all of the related signals into effect, the following equation results:

$\mathrm{CONF}_{\mathrm{i}-1}=$

$$
\begin{aligned}
& \left(\text { CONF }_{\mathrm{i}} \cdot \overline{\text { RIGHT_ED }_{\mathrm{i}}}\right. \\
& \left.+ \text { CARRY }_{\mathrm{i}+1} \cdot \mathrm{RIGHT}_{\mathrm{i}}\right) \cdot \overline{\mathrm{W}_{\mathrm{i}}} \\
& +\mathrm{CONF}_{\mathrm{i}} \cdot \mathrm{W}_{\mathrm{i}}
\end{aligned}
$$




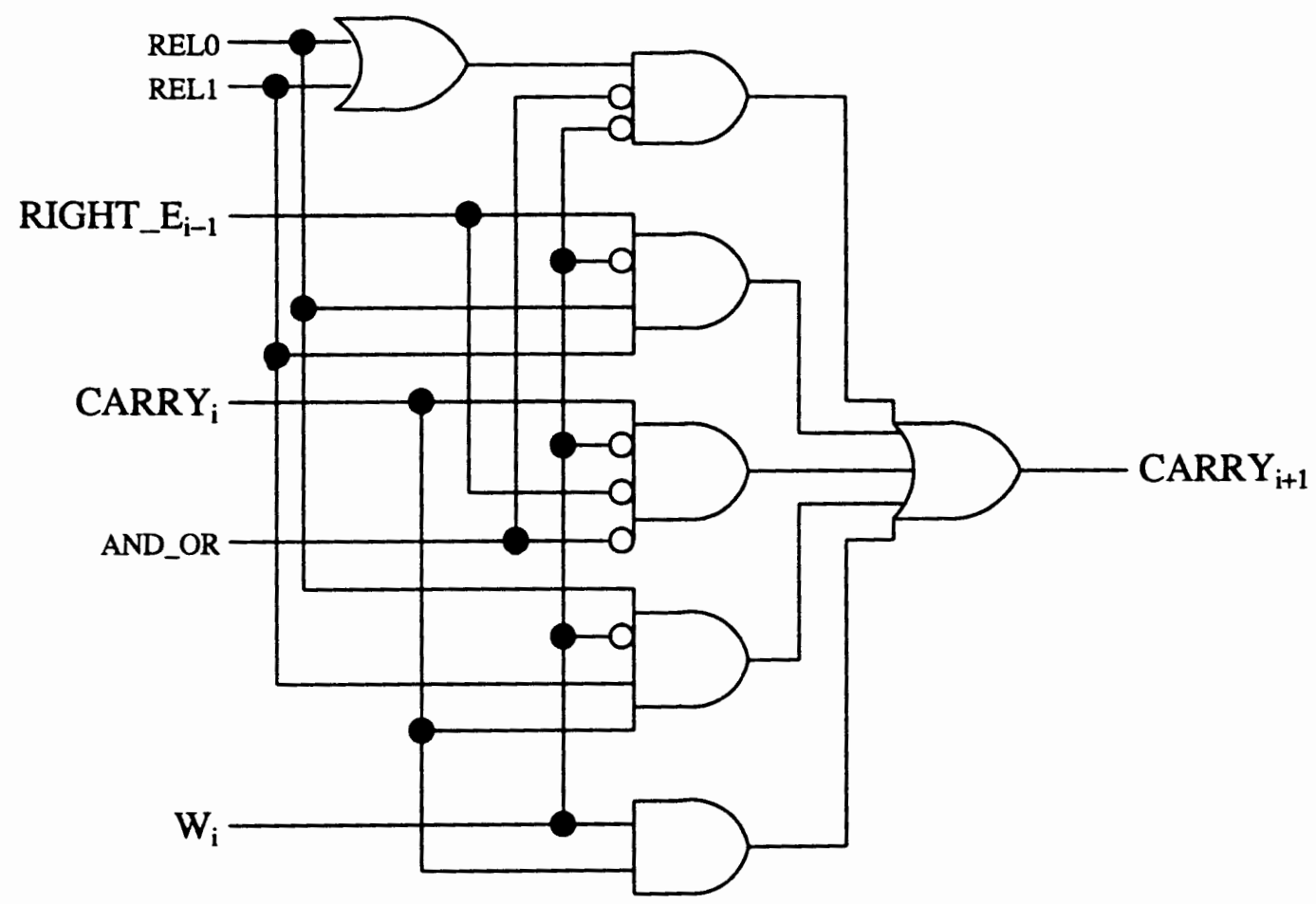

Figure 27. Gate - level realization of CARRY ${ }_{i}$.

The signal CONF $\mathrm{i}_{\mathrm{i}-1}$ is realized in the CCM2 in the logic circuitry in Figure 28.

VARIABLE $_{\mathrm{i}}$. When CONF $_{\mathrm{i}-1}$ equals 1 , this means that the relation is true for this variable, and the variable is specific, so VARIABLE $E_{i}$ is to be 1 . This IT is within a variable for which the relation on literals described by function "REL" is satisfied. 


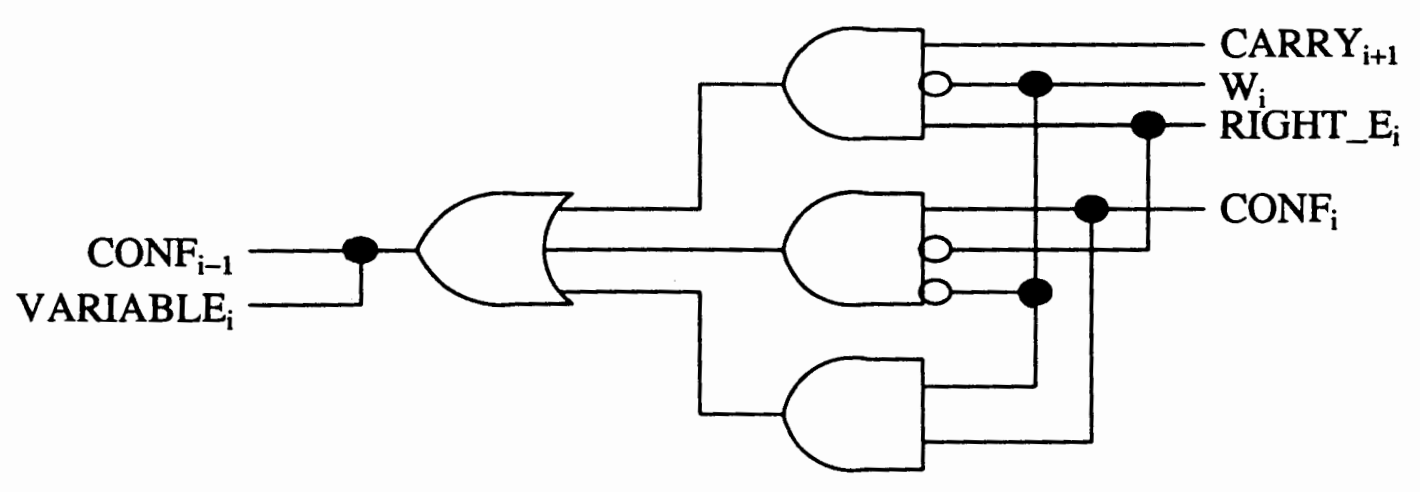

Figure 28. Gate-level realization of $\mathrm{CONF}_{\mathrm{i}-1}$.

$\mathrm{CNTO}_{\mathrm{i}}$. This signal is equal to $\mathrm{RELO} \mathrm{O}_{\mathrm{i}}+\mathrm{REL} 1_{\mathrm{i}}$ for OR-type relations and equal to $\mathrm{RELO}_{\mathrm{i}} \cdot \mathrm{REL1}_{\mathrm{i}}$ for AND-type relations. Since CONF $\mathrm{F}_{\mathrm{i}}$ indicates a specific position to be confirmed, if $\mathrm{CNT1}_{\mathrm{i}}$ is also true, one resultant cube will be generated. From this, the following equation results:

$\mathrm{CNT0}_{\mathrm{i}}=$

$$
\begin{aligned}
& \left(\mathrm{RELO}_{i} \cdot \mathrm{REL1}_{\mathrm{i}}\right) \cdot \mathrm{AND} \mathrm{OR} \\
& +\left(\mathrm{RELO}_{\mathrm{i}}+\mathrm{REL1}_{\mathrm{i}}\right) \cdot \overline{\mathrm{AND} O \mathrm{OR}}
\end{aligned}
$$

CNT1 $_{\mathrm{j}}$. This signal is used to generate the signal COUNT $\mathrm{i}_{\mathrm{i}}$ which counts the number of specific positions, producing the calculation of the total number of resultant cubes for the operation. $\mathrm{CNT1}_{\mathrm{i}}$ signalizes a one on the right edge of variables that are satisfied.

$\mathrm{CNT}_{\mathrm{i}}=$

$$
\text { RIGHT_E } E_{i} \cdot V_{\text {VARIABLE }}
$$

The two signals $\mathrm{CNTO}_{\mathrm{i}}$ and $\mathrm{CNT}_{\mathrm{i}}$ are realized in the CCM2 with the logic circuitry seen in Figure 29. 


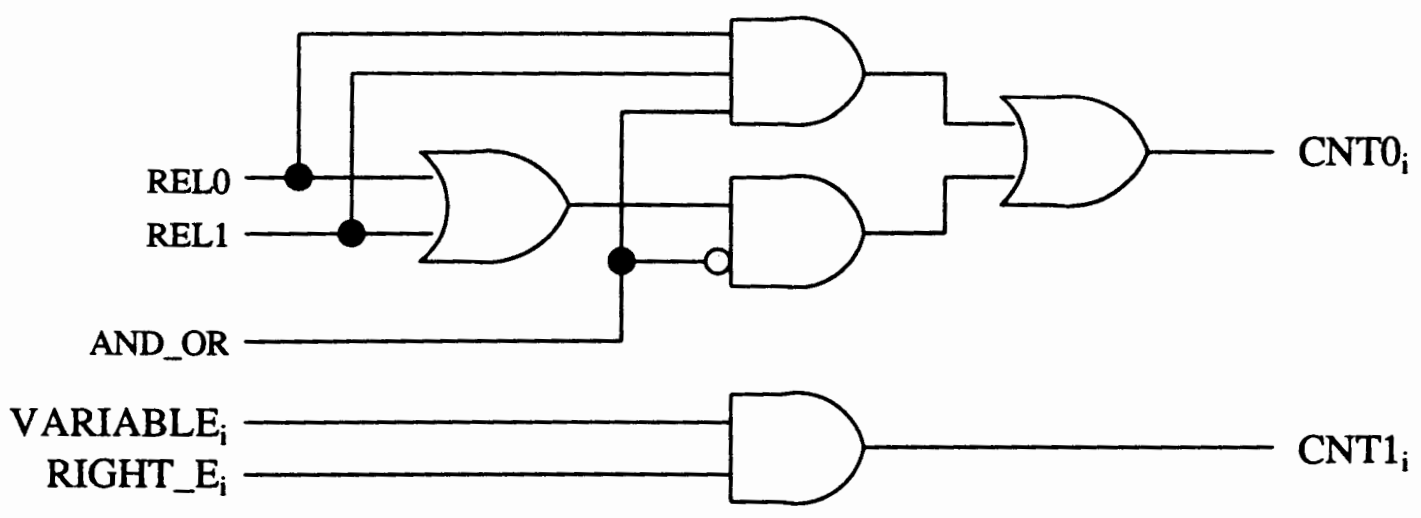

Figure 29. Gate - level realization of $C N T 0_{i}$ and $C N T 1_{i}$.

\section{State}

The block State $e_{i}$ within $\mathrm{IT}_{\mathrm{i}}$ is the control portion of an IT, containing a single positional state machine (PSM). The block State $_{i}$ is also responsible for handling the control signals that are sent to and received by the CU. Figure 30 shows the state assignment of the FSM for a single IT. The state of the IT is determined by two D flipflops with inputs named $\mathrm{EX} 0_{\mathrm{i}}$ and $\mathrm{EX} 1_{1}$, and outputs named STATE $0_{\mathrm{i}}$ and STATE $1_{i}$ respectively. The logic realization of block STATE in the CCM2 can be seen in Figure 31.

$\mathrm{EX0}_{\mathrm{i}}=$

BEFORE $_{i} \cdot$ VARIABLE $_{i} \cdot$ NEXT $_{i}$

$\mathrm{EX}_{1}=$

ACTIVE $_{\mathrm{i}} \cdot \overline{\text { CLEAR }}$

+ AFTER $_{i} \cdot \overline{\text { CLEAR }}$

+ BEFORE $_{i} \cdot \overline{\text { TESTATE }} \cdot \overline{\text { VARIABLE }} \cdot \overline{\text { NEXT }_{i}}$ 


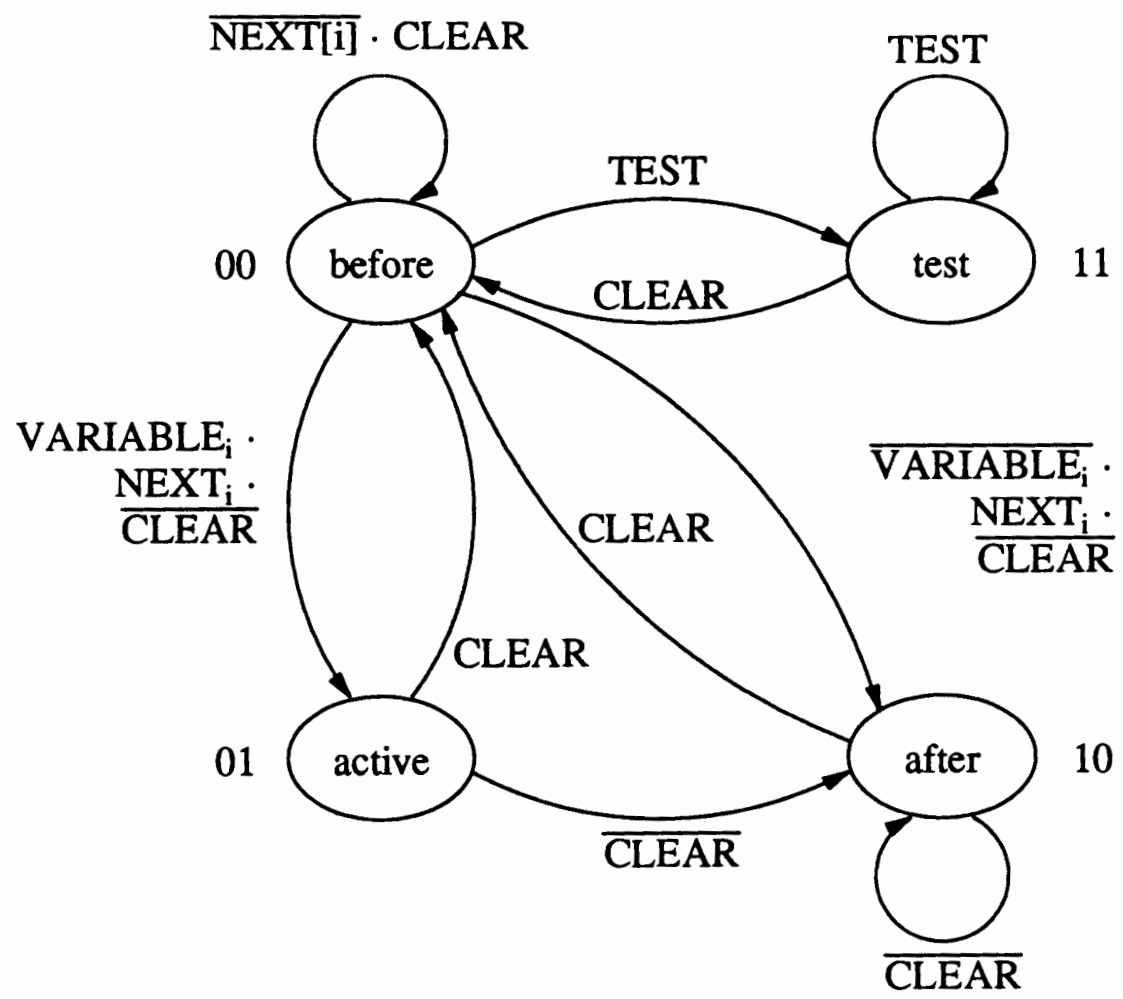

Figure 30. State diagram for $\mathrm{STATE}_{\mathrm{i}}$.

BEFORE $_{i}=\overline{\text { STATE0 }_{i}} \cdot \overline{\text { STATE }_{i}}$

ACTIVE $_{i}=$ STATE $_{i} \cdot \overline{\text { STATE } 1_{i}}$

AFTER $_{\mathrm{i}}=\overline{\text { STATE0 }_{\mathrm{i}}} \cdot$ STATE $_{\mathrm{i}}$

TESTATE $_{i}=\operatorname{STATE}_{\mathrm{i}} \cdot \mathrm{STATE}_{\mathrm{i}}$ 


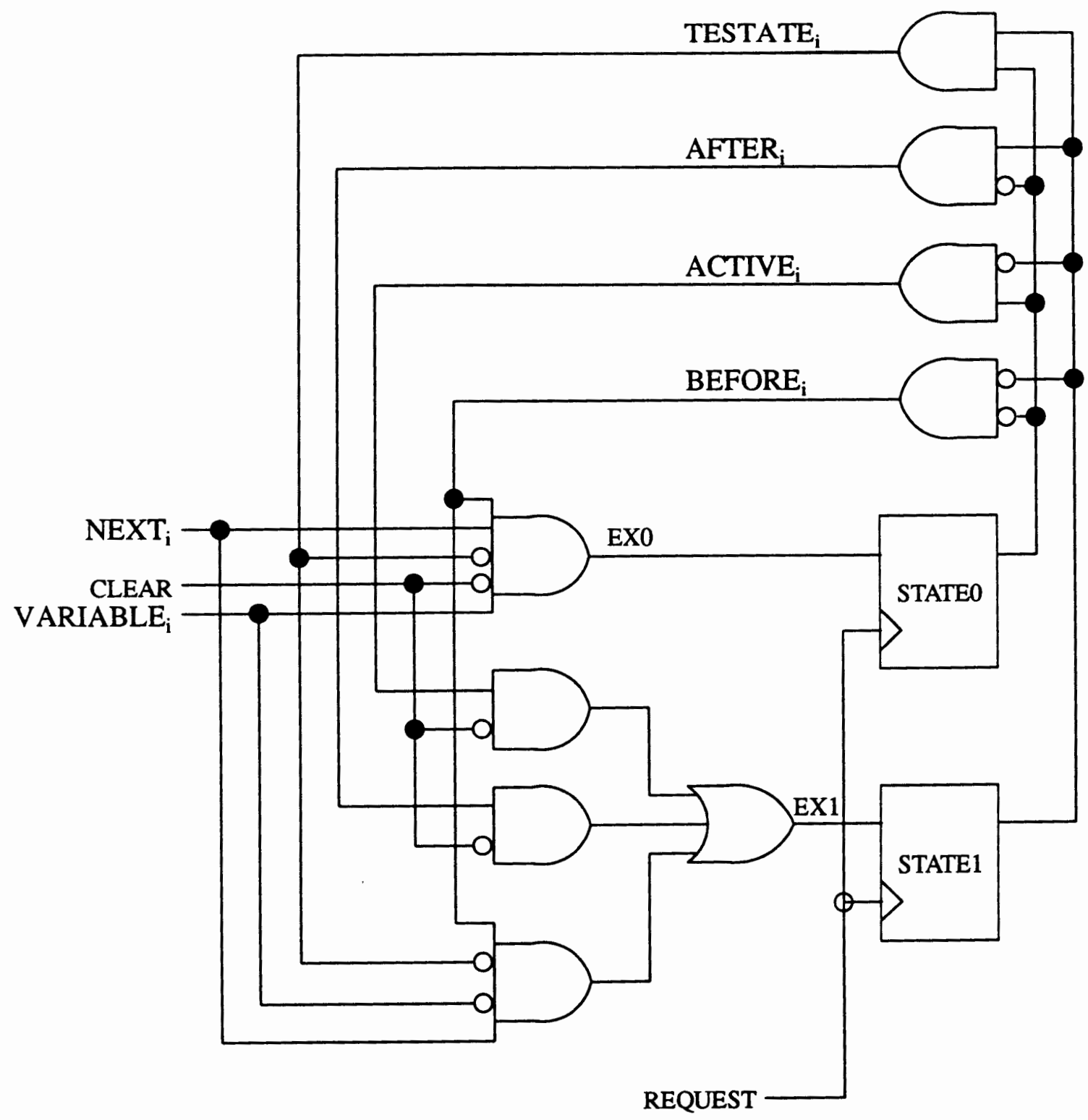

Figure 31. Gate - level realization of STATE ${ }_{i}$.

$\mathrm{NEXT}_{\mathrm{i}+1}$. Essentially, the position of the active literal moves from one specific literal to the next when the next resultant cube is going to be calculated. In order to avoid unnecessary delays, signal $\operatorname{NEXT}_{i}$ is propagated along the IT chain during the processing of input cubes, preparing ITs that are supposed to transit to the ACTIY'E state. Therefore, 
the transition to the ACTIVE state can be done immediately (that is, in the time determined by delays inside a single IT) with the coming of the active edge of the REQUEST clock. NEXT $T_{i}$ starts propagation to the right and is propagated until RIGHT_E $E_{i}=1$ and VARIABLE $E_{i}=1$. So on the last $\mathrm{IT}_{\mathrm{j}}$ of the next specific position $\mathrm{NEXT}_{j+1}$ will be 0 and in all previous $\mathrm{IT}_{\mathrm{i}} \mathrm{SNEXT}_{\mathrm{i}+1}$ will be 1.

$\operatorname{NEXT}_{i+1}=$

$\left(A_{C T I V E}+\right.$ NEXT $\left._{i} \cdot \overline{\left(\text { RIGHT_E }_{i} \cdot \text { VARIABLE }_{i}\right)}\right) \cdot \overline{W_{i}}$

$+\mathrm{NEXT}_{\mathrm{i}} \cdot \mathrm{W}_{\mathrm{i}}$

The signal NEXT $\mathrm{T}_{\mathrm{i}+1}$ is realized in the ILU by the logic circuitry shown in Figure 32.

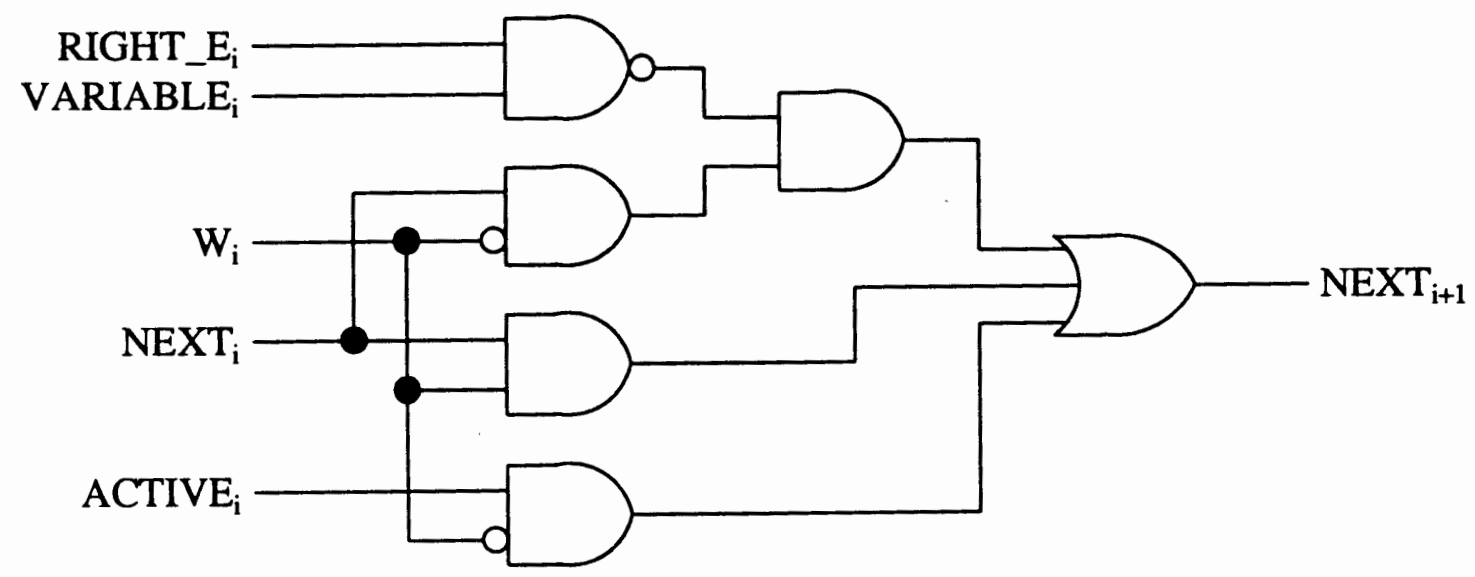

Figure 32. Gate - level realization of $\mathrm{NEXT}_{\mathrm{i}}$.

READY $_{\mathrm{i}} \cdot \operatorname{READY}_{\mathrm{i}}=1$ means that the rippling $\operatorname{NEXT}_{\mathrm{i}}$ signal has reached its destination and the next specific position is ready to be activated. The equation means: IT on the right edge of a specific variable has the active $\mathrm{NEXT}_{i}$ signal on its input and this happens after the REQUEST clock has been activated. When an $I_{i}$ becomes ACTIVE it sends the NEXT $_{i+1}$ signal, rippling to the next specific group of ITs (that is, ITs assigned to a specific variable). The NEXT $\mathrm{N}_{i}$ signal on the left side of the currently ACTIVE IT 
becomes low as soon as its low state ripples from the previously ACTIVE IT (now that IT is in the AFTER state, for which variable AFTER is true).

READY $_{\mathrm{i}}=\overline{\mathrm{REQUEST}} \cdot \mathrm{RIGHT}_{-} \mathrm{E}_{\mathrm{i}} \cdot \mathrm{NEXT}_{\mathrm{i}} \cdot$ VARIABLE $_{\mathrm{i}}$

READY $_{\mathrm{i}}$ is realized in the CCM2 by the logic seen in Figure 33.

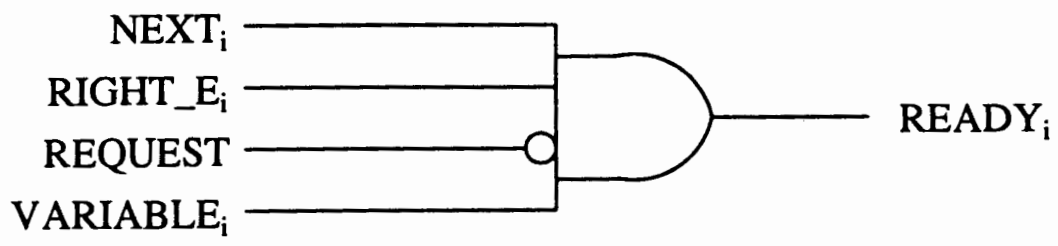

Figure 33. Gate-level realization of READY

$\mathrm{SLCT}_{\mathrm{i}}$. This two-bit variable is represented by the two signals $S L C T 0_{i}$ and $S L C T 1_{i}$, the least significant bit and most significant bit respectively. These signals are used to simply select the appropriate function: BEFORE, ACTIVE, or AFTER, to be performed by a given $\mathrm{T}$.

$S L C T 0_{i}=$ VARIABLE $_{i} \cdot$ PRIME STATE $_{i} \cdot \overline{\text { PRIME }}$

$\operatorname{SLCT} 1_{i}=\operatorname{STATE} 1_{i} \cdot \overline{\text { PRIME }}$

$\mathrm{SLCTO}_{\mathrm{i}}$ and $\mathrm{SLCT}_{\mathrm{i}}$ are realized in the ILU with the logic circuitry seen in Figure 34. 


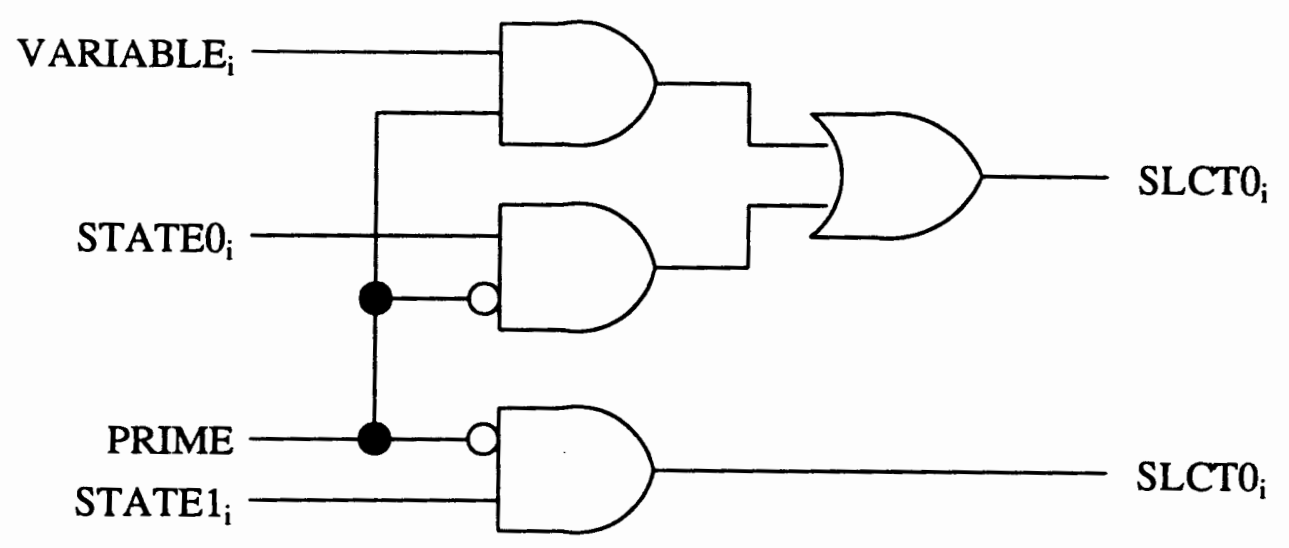

Figure 34. Gate-level realization of $S L C T 0_{i}$ and $\operatorname{SLCT1}_{i}$.

\section{Count}

In the CCM2, it is often necessary to count the occurrences of logical relations, such as counting contradictions (Epsilons) during the consensus operation. Each IT contributes either a zero or a one to the total count. The block COUNT is a successive circuit in each IT that accepts a predetermined input and either passes its input to its output, or produces the next element in the sequence at its output.

This successive circuit consists of a standard shift-register with an XOR gate as seen in Figure 35. This design is a standard binary incremental circuit commonly used in the feedback circuit of binary counters. A shift-register of length $M$ bits can generate a sequence of length $L$ where $L=2^{M}-1$. For most values of $M$, a maximal-length sequence can be generated with one two-input exclusive-OR gate in the feedback circuit.

The CCM2 counter must be able to count from zero up to the number of iterative cells. For $\mathrm{N}$ ITs, the count sequence length must be at least $\mathrm{N}+1$ in order to cover the case where the count is zero. The count is passed from one IT to the next through parallel signal lines. The first IT receives a fixed input count with the last IT resulting in 
the output of the total accumulated count.

The CCM2 counter has been implemented in this design, but is not used due to the purpose of our testing. The consensus operations which uses the resultant value of Count in its operations has been tested with a Hamming distance between the two input cubes to be less than or equal to one. It was mainly added here to help in the determination of the consumption of CLBs for the overall design. Future groups that will plan to work on the bus interface and implementing the pipelined version of the CCM2 will use this for delay purposes and preventing the occurrences of contradictions in the resultant cubes.

The equations and figures shown in this chapter represent the actual logic circuitry that has been programmed for each IT of the ILU for the CCM2 design. It is all interconnected as needed and was broken down in sections to aid in each signal's understanding. The following chapter discusses the interconnections of the ITs in the CCM2 design, how the ILU was programmed and downloaded to the devices, and how the demonstration board will be used to teach future classes about the design of the CCM2. 


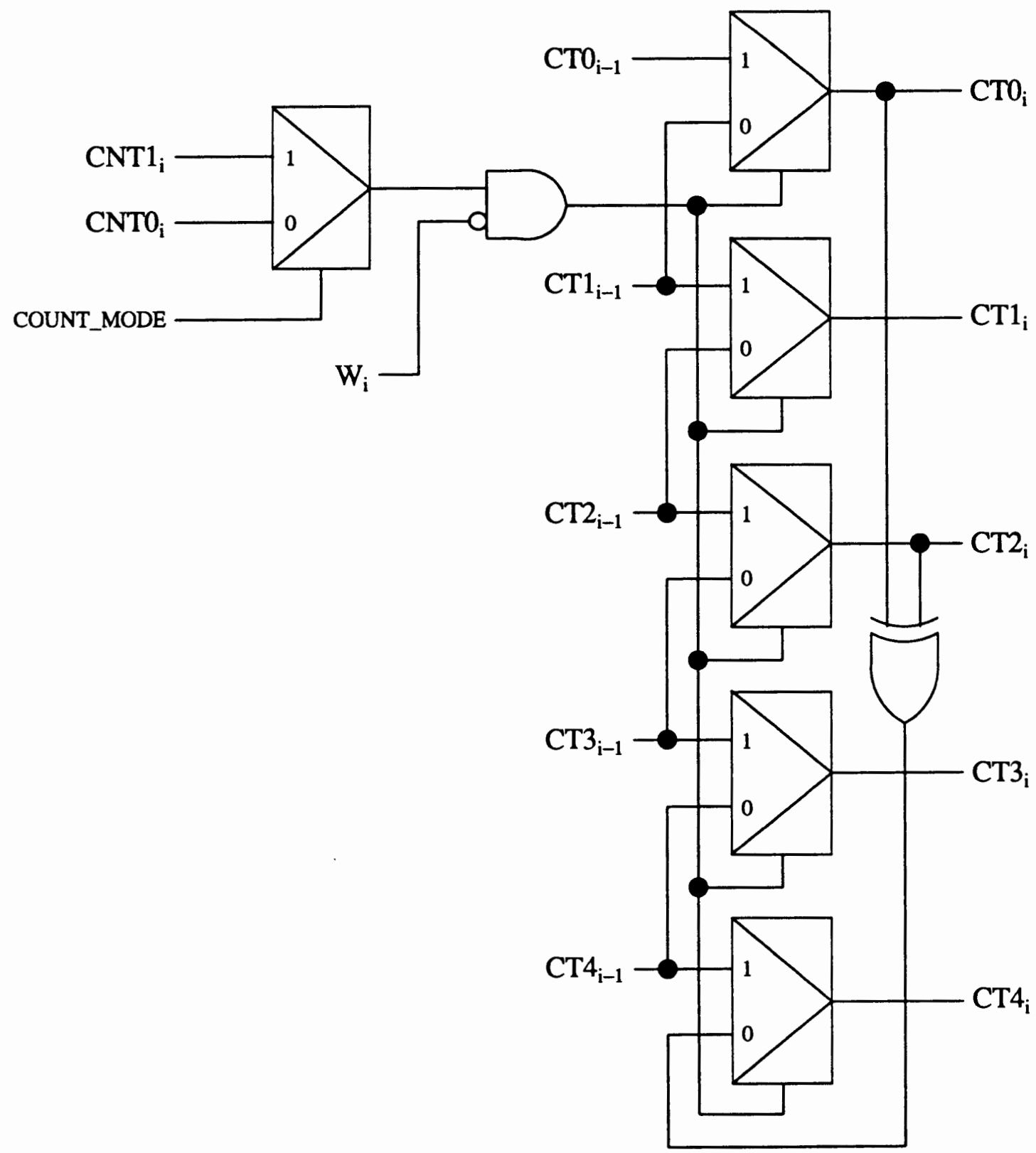

Figure 35. Gate - level realization of block COUNT. 


\section{CHAPTER V}

\section{REALIZATION OF THE ITERATIVE LOGIC UNIT}

The Iterative Logic Unit (ILU) of the Cube Calculus Machine II (CCM2) was chosen to be realized using Xilinx Logic Cell Arrays (LCAs), the pioneer in Field Programmable Gate Arrays (FPGAs). As discussed in Chapter II, this decision was made 'due to the lack of VLSI tools and the advantage of having the Xilinx XACT development system in our possession, which Doug Hall had brought with him from PCC.

This chapter presents the design of the physical ILU of the CCM2. It includes the reasoning behind the selection of the FPGA device, its programming and downloading strategies and examples of cube calculus operations executed within the ILU design.

\section{HARDWARE DESIGN}

When selecting a device, I had to estimate the size of the design and compare this with the LCA devices available for purchase. An ideal fit would consist of fitting a portion of the CCM2 design in an LCA device, while using one-half to two-thirds of the configurable elements per chip (Configurable Logic Blocks (CLBs) and I/O Blocks (I/OBs)) to leave room for revisions and expansion of future CCMs. I estimated that with our current design, 8 Iterative Cells (ITs) could be fit within a single XC3090 LCA, consuming approximately $75-80 \%$ of the available CLBs. With this estimation, an entire 32-bit Iterative Logic Unit (ILU) could fit within two LCA devices.

With the estimation from above, the Xilinx device chosen was the XC3090-50 PP175C. This particular LCA was chosen due to its logic density and the support range 
of our tools. It is the largest device of the XC3000 family, (our current tool set includes macros for the XC2000 and XC3000 families of Xilinx LCAs only) containing 320 CLBs and 144 I/OBs. With the estimated growth of the CCM design in future revisions and the machine's ability to be easily scaled dependent upon the application, it was decided that the XC3090 device would be optimal to support future growth and different projects, as well as using our project funding in the wisest fashion. The XC3090 is a 175 pin plastic PGA-grid device with a system clock speed grade of $50 \mathrm{MHz}$. We purchased four devices through Hamilton/Avnet Electronic in the Spring of 1992 at a cost of 202.50 dollars per device. As of May 16, 1994 these devices are obsolete for this speed grade. The same device with a system clock speed grade of $100 \mathrm{MHz}$ is available for 143.30 per device if purchased in the same manner through Marshall Industries here in Portland.

The design is laid out on a $10 \times 10$ in. circuit board recycled from a previous class project in Dr. Driscoll's EE585. The board has a voltage and ground plane with bananajack plugs for easy VCC and GND plug-in. The XC3090s are mounted in Zero Insertion Force (ZIF) sockets, piggy-backed upon standard 175 pin PGA grid sockets with leads specifically for wire-wrapping. The ZIF sockets are manufactured by AMP Inc. and run for 60.50 dollars apiece. I was able to obtain free samples, ending up with a total of three. The ZIF sockets are not manufactured with wire-wrapped pins, so I needed to obtain additional wire-wrapped sockets that I could piggy-back the ZIF sockets to. The standard wire-wrap PGA sockets are products of Mark-Eylet Inc., available for around 25.00 dollars and were also obtained for free, collecting a total of four.

The data registers are replaced by dip-switches, handling the inputs to the ILU. Inputs pertaining to the ILU that come from these switches include the input cubes A and B, RIGHT_E, W, REL, BEF, ACT and AFT. The instruction register (IR) also is realized as a bank of dip-switches. It provides inputs directly to the ILU as well as aiding in the 
operation of the control unit (CU). Figure 36 shows the general layout of the board, with the long rectangular labeled boxes representing the dip-switch banks.

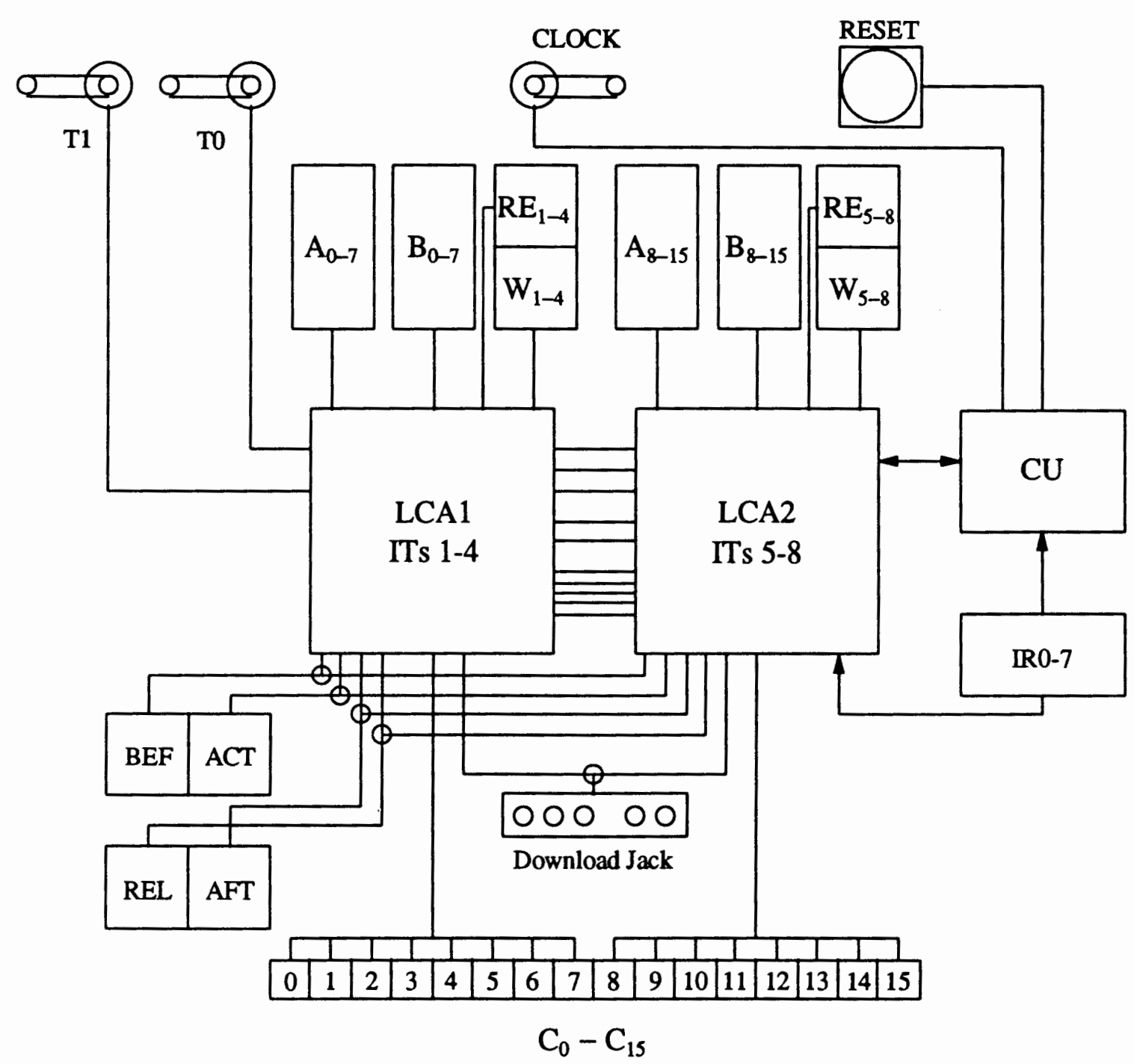

Figure 36. PC board layout of the CCM2.

Figure 37 shows each of the banks of dip-switches, the input value that each switch controls and the corresponding pin for the LCA device that the value is going to. A bottom view of the XC3090 LCA device with its grid-array layout may be seen in Figure 38. 
Additional control on the board includes the inputs TO and T1 which are discussed in Chapter IV and aid in controlling the values output to the LEDs across the bottom which are the values of the resultant cubes if $\mathrm{T} 0$ and $\mathrm{T} 1$ both equal zero. Different input values from $\mathrm{T} 0$ and $\mathrm{T} 1$ will produce different values received at the resultant cube outputs, enabling a user to look at different signals within the ILU. The values of TO and $\mathrm{T} 1$ and the corresponding values produced at the LEDs may be seen in TABLE VII in the previous chapter. To the top right portion of the board is the push-button switch RESET. This has a dual function for the design, acting as a reset to the $\mathrm{CU}$ to reset the unit from an initial unknown state to State 0 , and secondly as a reset to the LCA devices. This second reset of the LCAs will be discussed in a section to come on downloading of the design. 

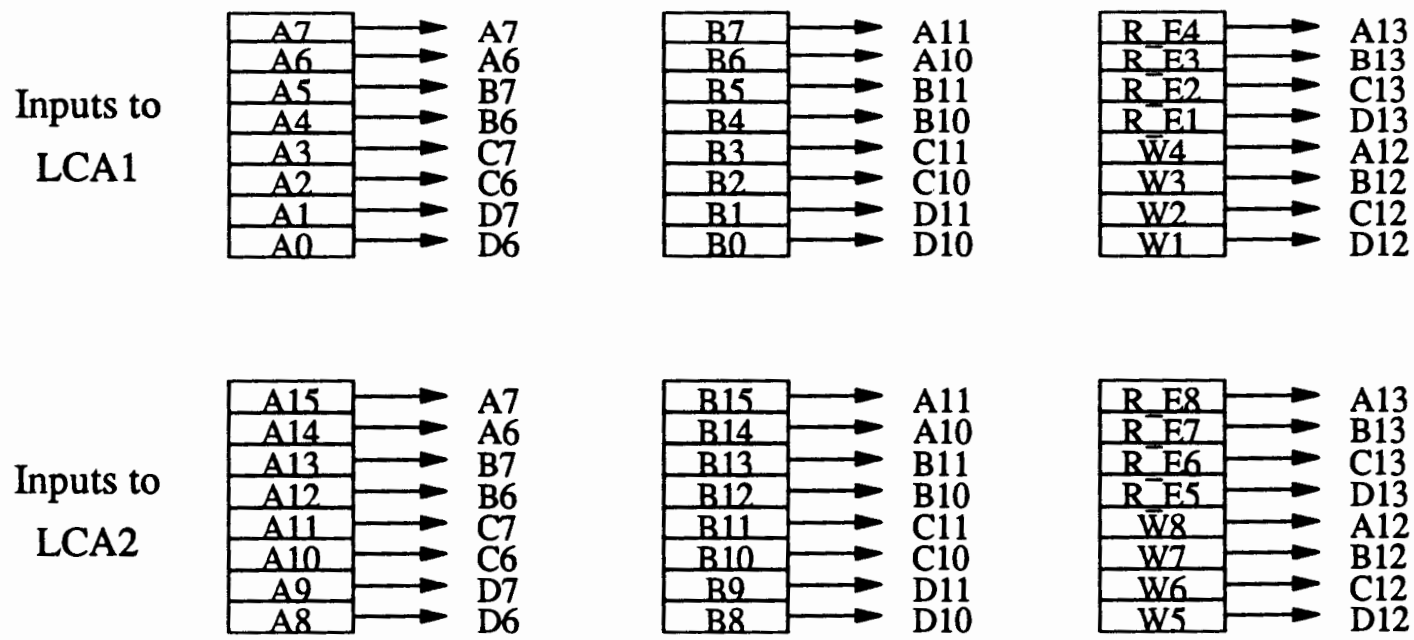

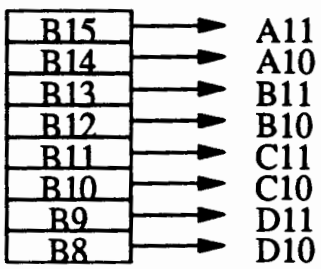

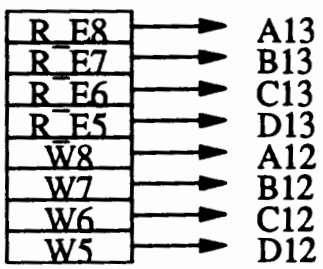

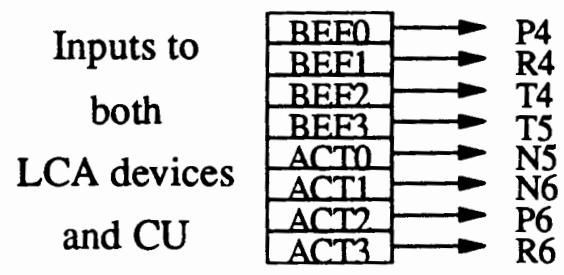

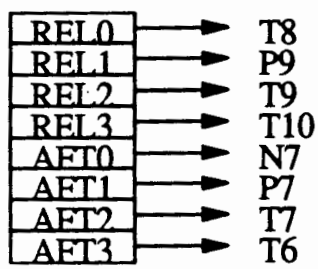

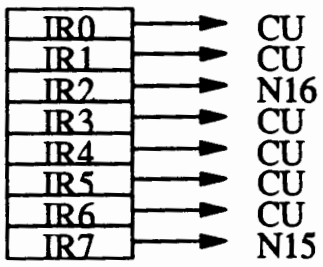

Figure 37. PC board dip-switch values.

The toggle switch at the top labeled CLOCK is the switch enabled to clock the CU for controlling the global signals of the ILU. The clock was implemented in this way so the user may step through a sequential or complex combinational cube calculus operation step by step. There are three LEDs located next to the CU that show the current state of the CU. Each time the switch is toggled, the CU progresses to the next state and that state is represented by the lights. The control unit was realized with a National Semiconductor GAL16V8 programmable device. Figure 21 in CHAPTER IV shows the state diagram for the CU, and the CUPL file that I wrote to program the CU may be found in Appendix A. When the ILU produces a resultant cube $C_{i}$, the values are seen at the bank of 16 LEDs across the bottom of the board. If the specific operation produces 
multiple resultant cubes, the first one is presented, then when the ILU produces the second cube $C_{2}$, it writes over the values of $C_{1}$ and so on. By hand clocking the $C U$, this enables the user to examine the resultant values at each stage of the progression of the operation.

The box below the LCAs labeled Download Pins is the connector where the Xilinx download cable is attached. As Figure 36 shows, I have these pins connecting to both devices. Since I have made the design in a regular fashion, each LCA device holds the identical design. Thus, I am able to download the same design into two devices at the same time. The process of downloading the design will be discussed in a section to come. 


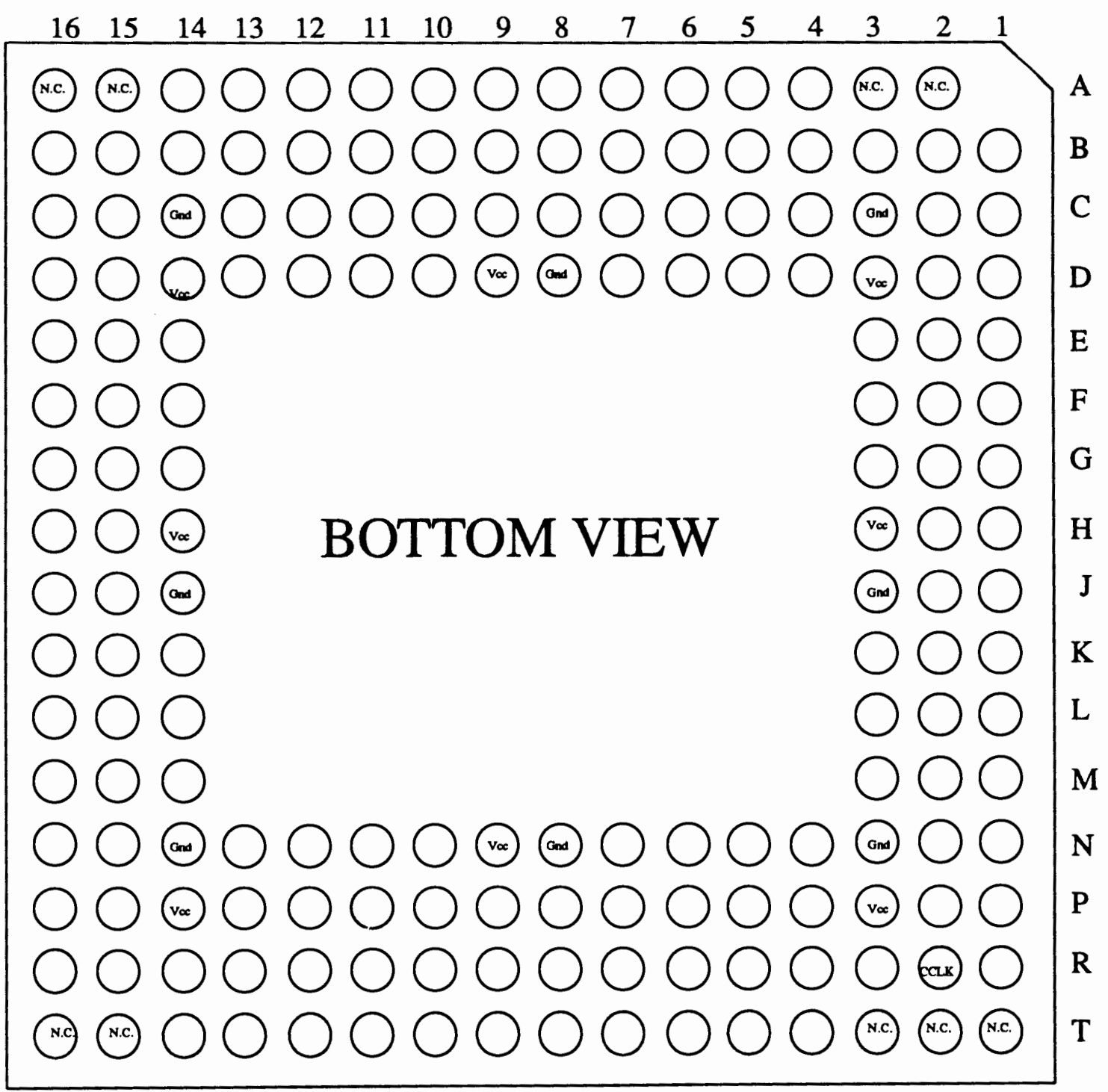

Figure 38. Xilinx XC3090-PP175 pin layout. 


\section{CONVERTING THE DESIGN}

The design of the CCM was first conceived by Louis Kida and Dr. Marek Perkowski. Classes of Dr. Perkowski then refined the machine and made many contributions toward the CCM2 design. An M-model was then made for the design of the ILU and CU, then simulated using Lsim included in the older Mentor tools. The design was then targeted to be realized using OCTOOLS, a VLSI package that is no longer supported here at PSU. The realization of the CCM2 sat idle until it was decided that I attempt the realization of the ILU using the Xilinx tools.

The design of the ILU implemented here is a collaboration of many students' ideas over the years. I have evaluated the design from past text and from the M-models created for the simulation of the design, and have added a few changes, safety precautions and have prepared the design for its mapping to the LCA devices. The actual schematic drawings for each iterative cell (IT) for the implemented design are shown in Chapter IV.

The ILU design was created using FutureNet DASH, a schematic capture package that was one of the first packages with an interface to the Xilinx tools. The design is hierarchical in design with the top-level drawing consisting of an ILU of four ITs. It is named 4ITILU.DWG and may be found on Dr. Perkowski's PC in his lab downstairs under the directory C:DAVIDICCM. The lowest-level drawing is a design of one IT and is named IT.DWG. It includes the internal logic of all the sections of the IT as discussed in detail in Chapter IV. After the single IT cell had been designed, I then created a "symbol" of the IT.DWG file. ITCELL.SYM is a block symbol with the necessary inputs and outputs corresponding to the IT.DWG file. When called, the ITCELL symbol appears in a block representation, replacing the entire schematic representation of IT.DWG. Thus the top-level design 4ITILU.DWG consists of four ITCELLs 
interconnected between its neighbors, and receiving inputs from the data registers and $\mathrm{CU}$, and producing outputs to communicate with the $\mathrm{CU}$ and produce resultant values.

Inputs and outputs are directed to the pins of the LCA device through adjusting the attributes of the IPAD and OPAD symbols in the schematic drawing. Each I/O from the design must be directed to a selected $\mathrm{I} / \mathrm{O}$ pin in the schematic design.

Once the design has been created in schematic form, it needs to first be converted to a Xilinx Netlist file (XNF). Chapter VI contains a short tutorial of a 7-segment decoder design that describes each operation in this conversion. In this chapter, I will present each conversion step within the design, but I will not go into great detail of the operation. The first step is to convert each of the drawing files into DCM files. This is a necessary step in the conversion of the drawing files and executed as follows: DCM 4ITLU.DWG, DCM IT.DWG. This operation creates the files 4ITILU.DCM and IT.DCM. Next, the PINC must be executed to create the FutureNet netlist-like design description files. By executing PINC 4ITILU.DCM and PINC IT.DCM, the files 4ITILU.PIN and IT.PIN are created.

The PIN files are now ready to be converted to XNF files by executing PIN2XNF. This program translates the PIN files into an XNF file. This is done by executing PIN2XNF upon the top-level file 4ITILU.PIN. PIN2XNF automatically finds all of the PIN files that correspond to the top-level file and associates them together, producing a single XNF file. In the case of this design, PIN2XNF -P 3090PG175-50 4ITILU.PIN was executed and the file 4ITILU.XNF was created. The switch -P 3090PG175-50 is used to target the design file toward the selected device. After the design has reached the XNF stage, it is now ready to be converted to an LCA file, which may be downloaded to the device. 
For our unique situation, I first had to convert the XNF file from an older version of Xilinx tools to the newer version. Our original tools date back to some of the earliest releases of Xilinx software. We have since obtained a more recent version of the tools, however, the latest tools did not come with an interface to any schematic capture tools. Thus, I converted the design from the FutureNet DASH schematic capture package with the older version of Xilinx software, then had to convert the files at the XNF level to the latest version of the Xilinx tools. This was done using XNFCVT -A 4ITILU.XNF ILU.XNF.

The design is now ready to be converted to an LCA file for downloading to the board. The command XNFMAP -P 3090PG175-50 ILU.XNF ILU.MAP, converts the XNF file to a partitioned MAP file. After the conversion to a MAP file has been made, the design must now be converted to an LCA file with the program MAP2LCA. The command MAP2LCA -P 3090PG175-50 ILU produces two files: ILU.LCA and ILU.CRF, a partitioned XNF netlist file and a record of logic mapped into CLBs and IOBs respectively. At this point, the design is ready to be automatically placed and routed using the APR program. APR uses the LCA design file ILU.LCA to automatically arrange the CLBs and IOBs, optimize the block and pin net assignments, and determine interconnection patterns to route the design and write the resulting design file to disk [60]. The command APR -W ILU ILUAPR places and routes the ILU.LCA file and writes the resultant LCA file to ILUAPR.LCA.

The APR placing process took 3 hours, 56 minutes and 52 seconds to place the design. The routing portion consumed 48 minutes and 21 seconds, producing a total APR time to be 4 hours, 45 minutes and 11 seconds. As a result, 131 of the available 320 CLBs were used and 74 of the available 144 IOBs were used. This leaves an enormous amount of resources within the device, proving that my initial estimate of fitting an ILU 
of 8 ITs within a single device is a realizable achievement. This will be given a close consideration in Chapter VII under my conclusions. TABLE VIII shows the extensions of the different files created during the design conversion process.

TABLE VIII

XILINX LCA CONVERSION FILE EXTENSIONS

\begin{tabular}{||l|l||}
\hline \hline \multicolumn{1}{|c|}{ Xilinx Files Created } \\
\hline Extension & \multicolumn{1}{|c|}{ File Contents } \\
\hline BAT & DOS batch file \\
DAT & Silos simulator input file \\
DIE & Binary description of LCA dies \\
EXE & DOS executable file \\
HLP & Silos simulator help file \\
MAC & Macro file \\
MSG & Silos simulator message file \\
OVL & Overlay file \\
PKG & Description of LCA packages \\
PRO & Commands executed at program startup (profiles) \\
XCT & Miscellaneous data file for XACT program \\
& \\
\hline \hline & \multicolumn{1}{|c|}{ Other XACT Created Files } \\
BDF & Binary file containing users LCA design \\
CUT & File used by CUT \& PASTE commands \\
DBK & Backup of editor commands in case of machine crash \\
LOG & Log of editor commands for design saved \\
LCA & File containing users LCA design \\
MAC & Macro file \\
OCF & Backup of CUT file overwritten by CUT command \\
OLF & Log of editor commands for design saved \\
OMF & Backup of macro file overwritten by CUTMACRO command \\
ODF & Old design file-backup of previous design file \\
PIC & Graphics print file used by PRINT DESIGN for plotters sup- \\
XNF & porting "Xilinx Metafile" format \\
& Xilinx Netlist Format file used as an interface to "external \\
& tools" such as schematic editors, simulators, and the Xilinx \\
\hline
\end{tabular}

After the APR process is finished, the design is ready to be viewed, modified, etc ... to the needs of the final design. By entering the Xilinx XACT Design Editor package 
(XDE), the design may be tailored in a number of ways. I have chosen to leave the design as it has been converted by the Xilinx programs executed in the above descriptions. At this point the design is ready to be downloaded to the CCM2 board. 


\section{DOWNLOADING THE DESIGN}

To download the ILU design to the Xilinx LCAs, the slave serial mode was chosen. This is the easiest method of downloading the LCA configuration to the device. The slave serial mode is the same mode used with the Xilinx Demo board discussed in Chapter VI, where serial data from the user-generated bit-stream file is downloaded in conjunction with a synchronizing input clock which drives the CCLK input of the LCA device. This bit-stream is generated in the XACT Design Editor.

To create the bit-stream file, I had to enter the Xilinx tools by typing XDE at the C:I prompt. When the main menu was entered, I selected the Programs menu, then selected the MakeBits option from the list of programs that appear. Once the MakeBits option had been selected, a new window appeared with several menu selections across the top. I chose the Config menu and selected MakeBits from the several choices that appeared. Once again several options appear that may be selected for added options for creating the bit-stream. I chose the Tie option which puts all unused interconnects in the device to a known value to reduce power consumption and on-chip noise. This option had a tendency to increase the timing of some nets and was not necessary for prototyping, but since speed was not a critical factor at this stage, I selected the Tie option for safety purposes. Then I selected Done to create the bit-stream and the bit-stream was generated.

When the bit-stream had been created, the message "Bitstream made, not written" appeared at the bottom of the screen. I then reentered the Config menu and selected the WriteBits option. When prompted for a filename, I pressed $\langle\mathrm{CR}\rangle$ to accept the default name of ILUAPR.BIT. The file is now saved to disk and ready to be downloaded to the device. 
To initiate the physical downloading of ILUAPR.BIT to the CCM2 board, the download cable was connected to the Xilinx key which was attached to the parallel port LPT1 of the host PC. The other end was then connected to the physical CCM2 board to the download cable jack which I have implemented. In the design of the CCM2 board, I installed a jack that fit the Xilinx download cable's keyed connector. Figure 36 shows the placement of the jack on the CCM2 board. TABLE IX shows the download cable's pin/color orientation and respective LCA signals that it must be connected to. Power must then be applied to the board via the VCC/GND banana jacks at the top of the board. I then entered the Misc menu and selected the Port option, where the program must be told which port the download cable is attached (LPT1 for our current setup). The port was then initialized, returning a "Printer LPT1 initialized" upon the successful initialization. At that point I entered the Download menu and selected Download. 
TABLE IX

\section{XILINX DOWNLOAD CABLE PIN ASSIGNMENTS}

\begin{tabular}{|l|l|l|l|}
\hline \multicolumn{3}{|c|}{ Download Header Target Connector } \\
\hline Pin & Color & Signal & Connect To \\
\hline \hline 1 & Red & VCC & Target System VCC \\
\hline 2 & Black & GND & Target System Ground \\
\hline 3 & Key & & \\
\hline 4 & Yellow & CCLK & Target LCA Configuration Clock Pin \\
\hline 5 & Blue & D/P & Target LCA Done/Program Pin \\
\hline 6 & Green & DIN & Target LCA Data In Pin \\
\hline
\end{tabular}

Upon choosing the Download option, the program prompted me to reset the LCA by pressing the Reset button on the board. After pressing Reset, I then pressed $<\mathrm{CR}>$ on the host PC and the message "Programming ..." appeared. After only 0.88 seconds, the message " 'done' signal now high" appeared, verifying a successful download to the devices. The serial configuration bit-stream must be available at the DIN/D0 input a short setup time before each rising CCLK edge. The file ILUAPR.BIT was 8046 bytes in size, making the download time of 0.88 seconds quite insignificant in contrast to the time to design and convert the files to LCA files. For a typical design, one could estimate that for minor changes within a design, a user could alter the design and have it downloaded to an LCA device within a single work day. This highly increases the time-to-market period over ASIC devices. 
The following section describes each signal important to the downloading and functionality of the Xilinx devices. Each signal/pin is described in detail, and a description of what I did for this specific design is included.

\section{Permanently Dedicated Pins}

VCC. Connections to the nominal $+5 \mathrm{~V}$ supply voltage. All must be connected.

GND. Connections to ground. All must be connected.

PWRDWN. A Low on this CMOS-compatible input stops all internal activity, but retains configuration. All flip-flops and latches are reset, all outputs are 3-stated, and all inputs are interpreted as High, independent of their actual level. While $\overline{\text { PWRDWN }}$ is Low, VCC may be reduced to any value greater than $2.3 \mathrm{~V}$. When $\overline{\text { PWRDWN }}$ returns High, the LCA becomes operational with DONE Low for two cycles of the internal 1-MHz clock. During configuration, $\overline{\text { PWRDWN }}$ must be High. If not used, $\overline{\text { PWRDWN }}$ must be tied to VCC. In the case of the CCM2 design I have in fact tied this high.

RESET. This is an active Low input which has three functions. Prior to the start of configuration, a Low input will delay the start of the configuration process. An internal circuit senses the application of power and begins a minimal time-out cycle. When the time-out and $\overline{\text { RESET }}$ are complete, the levels of the $M$ lines are sampled and configuration begins. If $\overline{\text { RESET }}$ is asserted during a configuration, the LCA device is reinitialized and restarts the configuration at the termination of $\overline{\text { RESET. If } \overline{R E S E T}}$ is asserted after configuration is complete, it provides a global asynchronous reset of all IOB and CLB storage elements of the LCA device. In the CCM2 design, this signal is controlled by the large black push-button at the top of the board. The inverse signal of $\overline{\text { RESET }}$ is used to reset the CU back to State 0 .

CCLK. During configuration, Configuration Clock is an output of an LCA in 
Master mode or Peripheral mode, but an input in Slave mode. During Readback, CCLK is a clock input for shifting configuration data out of the LCA. CCLK Drives dynamic circuitry inside the LCA. The Low time may, therefore, not exceed a few microseconds. When used as an input, CCLK must be "parked High". An internal pull-up resistor maintains High when the pin is not being driven. The corresponding pin for CCLK is tied to the download cable jack on the CCM2 board located right below the LCA devices.

DONE/ $\overline{\text { PROG }}(\mathrm{D} / \overline{\mathrm{P}})$. Done is an open-drain output, configurable with or without an internal pull-up resistor. At the completion of configuration, the LCA circuitry becomes active in a synchronous order: DONE is programmed to go active High one cycle either before or after the outputs go active. Once configuration is done, a High-to-Low transition of this pin will cause an initialization of the LCA and start a reconfiguration. This signal is also controlled by the download process and is connected to the download cable jack below the LCA devices.

M0/RTRIG. As Mode 0, this input and M1, M2 are sampled before the start of configuration to establish the configuration mode to be used. A Low-to-High input transition, after configuration is complete, acts as a Read Trigger and initiates a Readback of configuration and storage-element data clocked be CCLK. By selecting the appropriate Readback option when generating the bit-stream, this operation may be limited to a single Readback, or be inhibited altogether. In the CCM2 design, I have tied this signal high since the slave-mode download configuration is used.

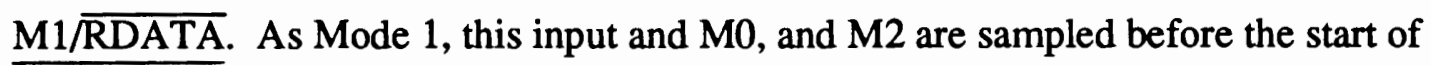
configuration to establish the configuration mode to be used. If Readback is never used, M1 can be tied directly to GND or VCC. If Readback is never used, M1 must use a 5-k $\Omega$ resistor to ground or VCC, to accommodate the $\overline{\text { RDATA }}$ output. As an active Low Read Data, after configuration is complete, this pin is the output of the Readback data. This 
signal is also tied high for the CCM2 slave-mode download configuration.

User I/O Pins That Can Have Special Functions.

M2. During configuration, this input has a weak pull-up resistor. Together with M0 and $\mathrm{M} 1$, it is sampled before the start of configuration to establish the configuration mode to be used. After configuration, this pin is a user-programmable $1 / O$ pin. In the design of the CCM2, I have decided to tie this pin high since the download configuration is done in slave mode. Since this user $\mathrm{I} / \mathrm{O}$ is not needed for this smaller design, no problems will be encountered. If the design is doubled in size, this pin might be needed as an I/O.

HDC. During configuration, this output is held at a High level to indicate that configuration is not yet complete. After configuration, this pin is a user-programmable I/O pin. This pin has not been used as an I/O in the design, so it has been left alone.

$\overline{\mathrm{LDC}}$. During configuration, this output is held at a Low level to indicate that the configuration in not yet complete. After configuration, this pin is a user-programmable I/O pin. $\overline{\mathrm{LDC}}$ is particularly useful in Master mode as a Low enable for an EPROM, but it must then be programmed as a High after configuration. This pin has been left alone since it is not needed as $\mathrm{I} / \mathrm{O}$ after configuration.

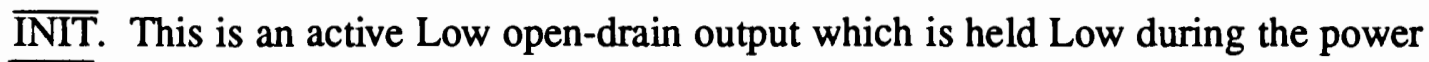
stabilization and internal clearing of the configuration memory. It can be used to indicate status to a configuring microprocessor or, as a wire-AND of several slave mode devices, a hold-off signal for a master mode device. After configuration this pin becomes a userprogrammable I/O. This pin has been left alone.

$\underline{B C L K I N}$. This is a direct CMOS level input to the alternate clock buffer (Auxiliary Buffer) in the lower right corner. This pin has not been used in the design of the CCM2.

XTL1. This user I/O pin can be used to operate as the output of an amplifier driving 
an external crystal and bias circuitry. The crystal option has not been used in this design, so the pin has been left alone.

XTL2. This user I/O pin can be used as the input of an amplifier connected to an external crystal and bias circuitry. The I/O Block is left unconfigured. The oscillator configuration is activated by routing a net from the oscillator buffer symbol output and by the MakeBits program. The crystal option has not been used in this design, so the pin has been left alone.

$\overline{\mathrm{CS} 0}, \overline{\mathrm{SC} 1}, \mathrm{CS} 2, \overline{\mathrm{WS}}$. These four inputs represent a set of signals, three active Low and one active High, that are used to control configuration-data entry in the Peripheral mode. Simultaneous assertion of all four inputs generates a Write to the internal data buffer. The removal of any assertion clocks in the D0-D7 data. In Master-Parallel mode, $\overline{\mathrm{WS}}$ and CS2 are the A0 and A1 outputs. After configuration, these pins are userprogrammable I/O pins. These pins were not needed for the configuration in slave mode and were left alone.

$\overline{\mathrm{RCLK}}$. During Master parallel mode configuration $\overline{\mathrm{RCLK}}$ represents a "read" of an external dynamic memory device (normally not used). After configuration is complete, this pin becomes a user-programmed I/O pin.

RDY/ $\overline{\text { BUSY }}$. During Peripheral parallel mode configuration this pin indicates when the chip is ready for another byte of data to be written to it. After configuration is complete, this pin becomes a user-programmed I/O pin.

D0-D7. This set of eight pins represents the parallel configuration byte for the parallel Master and Peripheral modes. After configuration is complete, they are userprogrammed I/O pins. D0 was the only pin that was needed during the download configuration. It acts as the DIN pin where the serial bit-stream generated by the host system is accepted. It is connected to the download cable jack on the board located 
below the LCA devices.

A0-A15. During Master Parallel mode, these 16 pins present an address output for a configuration EPROM. After configuration, they are user-programmable I/O pins.

DIN. During Slave or Master Serial configuration, this pin is used as a serial-data input. In the Master or Peripheral configuration, this is the Data 0 input. After configuration is complete, this pin becomes a user-programmed I/O pin. This pin is connected to the download cable jack on the board below the LCA devices.

DOUT. During configuration this pin is used to output serial-configuration data to the DIN pin of a daisy-chained slave. After configuration is complete, this pin becomes a user-programmed I/O pin. This pin was not implemented since the design is not configured between the two devices in a daisy-chained manner.

TCLKIN. This is a direct CMOS-level input to the global clock buffer. This pin can also be configured as a user programmable I/O pin. However, since TCLKIN is the preferred input to the global clock net, and the global clock net should be used as the primary clock source, this pin is usually the clock input to the chip. This I/O was not used since the design does not use the global clock net option.

Unrestricted User I/O Pins.

I/O. An I/O pin may be programmed by the user to be an input or an Output pin following configuration. All unrestricted I/O pins, plus the special pins mentioned on the following page, have a weak pull-up resistor of $50 \mathrm{k} \Omega$ to $100 \mathrm{k} \Omega$ that becomes active as soon as the device powers up, and stays active until the end of configuration. TABLE $\mathrm{X}$ shows all of the pins in the XC3090 device, the respective input or output signal at that pin, and how I have assigned the user-configurable I/O pins for the CCM2 design. 
TABLE $\mathbf{X}$

XC3090 PIN ASSIGNMENTS

\begin{tabular}{|c|c|c|}
\hline \multicolumn{3}{|c|}{ XC3090 175-Pin Plastic PGA Pinouts } \\
\hline PGA Pin \# & Pin Description & CCM2 Signals \\
\hline$\overline{\mathrm{A} 2}$ & N.C. & \\
\hline$\overline{\mathrm{A} 3}$ & N.C. & \\
\hline A4 & T/O & \\
\hline A5 & $\mathrm{I} / \mathrm{O}$ & \\
\hline A6 & I/O & A6 \\
\hline A7 & IOO & A7 \\
\hline A8 & I/O & \\
\hline A9 & I/O & \\
\hline A10 & 1/O & B6 \\
\hline A11 & I/O & B7 \\
\hline A12 & I/O & W4 \\
\hline A13 & I/O & RIGHT_E4 \\
\hline A14 & I/O & \\
\hline A15 & N.C. & \\
\hline A16 & N.C. & \\
\hline$\overline{\mathrm{B} 1}$ & IO & RIGHT Ei-1 \\
\hline$\overline{\mathbf{B} 2}$ & PWRDN & VCC \\
\hline B3 & $\mathrm{I} / \mathrm{O}$ & \\
\hline $\bar{B} 4$ & $\mathrm{I} / \mathrm{O}$ & \\
\hline$\overline{\mathrm{B} 5}$ & $\mathrm{I} / \mathrm{O}$ & \\
\hline B6 & 1/O & $\overline{\mathrm{A} 4}$ \\
\hline$\overline{\mathrm{B} 7}$ & I/O & $\overline{\mathrm{A} 5}$ \\
\hline B8 & I/O & \\
\hline B9 & $1 / 0$ & \\
\hline B10 & IO & $\overline{\mathrm{B} 4}$ \\
\hline$\overline{B 11}$ & $\overline{\mathrm{DO}}$ & $\overline{\mathrm{B} 5}$ \\
\hline B12 & I/O & W3 \\
\hline$\overline{\mathrm{B} 13}$ & I/O & RIGHT_E3 \\
\hline$\overline{B 14}$ & M1 - RDATA & VCC \\
\hline$\overline{\mathrm{B} 15}$ & M0-RTRIG & VCC \\
\hline B16 & I/O & \\
\hline C1 & A8-1/O & \\
\hline
\end{tabular}




\begin{tabular}{|c|c|c|}
\hline \multicolumn{3}{|c|}{ XC3090 175-Pin Plastic PGA Pinouts } \\
\hline PGA Pin \# & Pin Description & CCM2 Signals \\
\hline $\mathrm{C} 2$ & A9-I/O & \\
\hline C3 & GND & GND \\
\hline $\mathrm{C4}$ & T/O & \\
\hline C5 & I/O & \\
\hline C6 & I/O & A2 \\
\hline C7 & I/O & A3 \\
\hline C8 & $\mathrm{I} / \mathrm{O}$ & \\
\hline C9 & I/O & \\
\hline C10 & T/O & B2 \\
\hline C11 & I/O & $\overline{B 3}$ \\
\hline C12 & I/O & W2 \\
\hline C13 & I/O & RIGHT_E2 \\
\hline C14 & GND & GND \\
\hline C15 & M2 - I/O & VCC \\
\hline C16 & I/O & \\
\hline & 111 IC & \\
\hline D1 & A11 - I/O & \\
\hline D2 & $1 / 0$ & \\
\hline$\overline{\mathrm{D} 3}$ & VCC & VCC \\
\hline$\overline{\mathrm{D} 4}$ & TCLKIN - I/O & \\
\hline D5 & I/O & \\
\hline D6 & T/O & A0 \\
\hline D7 & I/O & A1 \\
\hline D8 & GND & GND \\
\hline D9 & VCC & VCC \\
\hline D10 & T/O & B0 \\
\hline D11 & 1/O & BT \\
\hline$\overline{D 12}$ & T/O & W1 \\
\hline D13 & T/O & RIGHT_EI \\
\hline D14 & VCC & VCC \\
\hline D15 & T/O & \\
\hline D16 & $\overline{L D C}-\mathrm{I} / \mathrm{O}$ & \\
\hline & & \\
\hline E1 & A7 - I/O & \\
\hline E2 & $1 / 0$ & \\
\hline E3 & A10-I/O & \\
\hline E14 & HDC-I/O & \\
\hline E15 & I/O & \\
\hline E16 & $\mathrm{I} / \mathrm{O}$ & \\
\hline & & \\
\hline F1 & $1 / 0$ & \\
\hline $\bar{F} 2$ & A12 - I/O & \\
\hline
\end{tabular}




\begin{tabular}{|c|c|c|}
\hline \multicolumn{3}{|c|}{ XC3090 175-Pin Plastic PGA Pinouts } \\
\hline PGA Pin \# & Pin Description & CCM2 Signals \\
\hline$\overline{\overline{F 3}}$ & $\overline{\mathrm{I} / \mathrm{O}}$ & \\
\hline F14 & T/O & \\
\hline F15 & I/O & \\
\hline F16 & IOO & \\
\hline G1 & I/O & CONFi-1 \\
\hline G2 & $\overline{\mathrm{I} / \mathrm{O}}$ & \\
\hline G3 & I/O & \\
\hline G14 & 1/O & \\
\hline G15 & I/O & \\
\hline G16 & $1 / 0$ & CONFi \\
\hline $\mathrm{H} 1$ & A6.IIO & \\
\hline & 112 & \\
\hline & $A 15-1 / U$ & \\
\hline H3 & VCC & VCC \\
\hline H14 & VCC & VCC \\
\hline$\overline{\mathrm{H} 15}$ & $\mathrm{INT}-\mathrm{I} / \mathrm{O}$ & \\
\hline H16 & $\mathrm{I} / \mathrm{O}$ & CARRYi+1 \\
\hline $\mathrm{J} 1$ & $\mathrm{I} / \mathrm{O}$ & CARRYi \\
\hline $\mathrm{J} 2$ & IO & NEXTi \\
\hline $\mathrm{J} 3$ & GND & GND \\
\hline $\mathrm{J} 14$ & GND & GND \\
\hline J15 & I/O & \\
\hline J16 & I/O & NEXTi+1 \\
\hline & & \\
\hline K1 & A5 - I/O & \\
\hline $\mathrm{K} 2$ & $\mathrm{~A} 14-\mathrm{I} / \mathrm{O}$ & \\
\hline$\overline{\mathrm{K}} 3$ & $\mathrm{I} / \mathrm{O}$ & CT1i-1 \\
\hline K14 & I/O & CTli \\
\hline K15 & 1/O & CT2i \\
\hline K16 & $1 / 0$ & CT3i \\
\hline C1 & ग/0 & CT2i-1 \\
\hline $\mathrm{L} 2$ & $\mathrm{~A} 4-\mathrm{D} / \mathrm{O}$ & \\
\hline $\mathrm{L} 3$ & I/O & CT3i-1 \\
\hline L14 & I/O & \\
\hline L15 & $\mathrm{I} / \mathrm{O}$ & CT4i \\
\hline L16 & $\mathrm{I} / \mathrm{O}$ & CT5i \\
\hline M1 & & \\
\hline $\mathrm{M} 2$ & A4-I/O & CT41-1 \\
\hline
\end{tabular}




\begin{tabular}{|c|c|c|}
\hline \multicolumn{3}{|c|}{ XC3090 175-Pin Plastic PGA Pinouts } \\
\hline PGA Pin \# & Pin Description & CCM2 Signals \\
\hline$\overline{\mathrm{M3}}$ & A1 - CS2-I/O & \\
\hline M14 & $1 / 0$ & CLEAR \\
\hline M15 & 1/O & \\
\hline M16 & I/O & REQUEST \\
\hline $\mathrm{N1}$ & $\overline{A 3-110}$ & \\
\hline $\mathrm{N} 2$ & $1 / 0$ & CT5i-1 \\
\hline N3 & GND & GND \\
\hline $\mathrm{N} 4$ & DOUT - I/O & \\
\hline N5 & I/O & ACT0 \\
\hline N6 & I/O & ACT1 \\
\hline N7 & I/O & AFT0 \\
\hline N8 & GND & GND \\
\hline N9 & VCC & VCC \\
\hline N10 & IDO & TESTO \\
\hline N11 & IVO & C5 \\
\hline N12 & I/O & \\
\hline N13 & D7-1/O & \\
\hline N14 & GND & GND \\
\hline N15 & $\mathrm{T} / \mathrm{O}$ & PRIME \\
\hline N16 & I/O & COUNT_MODE \\
\hline$\overline{\mathbf{P 1}}$ & $\mathrm{A} 2-1 / 0$ & \\
\hline$\overline{\mathbf{P} 2}$ & $\mathrm{~A} 0$ - WS - I/O & \\
\hline P3 & VCC & VCC \\
\hline P4 & T/O & BEF0 \\
\hline P5 & RDY/BUSY - RCLK - I/O & \\
\hline P6 & $\mathrm{I} / \mathrm{O}$ & ACT2 \\
\hline P7 & $\mathrm{I} / \mathrm{O}$ & AFT1 \\
\hline$\overline{\mathrm{P} 8}$ & $\mathrm{D} 3-1 / 0$ & \\
\hline P9 & 1/O & RELT \\
\hline P10 & I/O & TEST1 \\
\hline P11 & $\mathrm{T} / \mathrm{O}$ & $\mathrm{C6}$ \\
\hline P12 & D6-1/O & \\
\hline P13 & $\mathrm{I} / \mathrm{O}$ & $\mathrm{CO}$ \\
\hline P14 & VCC & VCC \\
\hline P15 & XTAL (IN) - I/O & \\
\hline P16 & $\mathrm{I} / \mathrm{O}$ & AND_OR \\
\hline R1 & TOO & READYi \\
\hline $\mathrm{R} 2$ & CCLK & \\
\hline$\overline{\mathrm{R} 3}$ & D0-I/O & \\
\hline
\end{tabular}




\begin{tabular}{|c|c|c|}
\hline \multicolumn{3}{|c|}{ XC3090 175-Pin Plastic PGA Pinouts } \\
\hline PGA Pin \# & $\begin{array}{l}\text { Pin Description } \\
\end{array}$ & CCM2 Signals \\
\hline$\overline{\overline{\mathrm{R} 4}}$ & $\overline{\mathrm{I} / \mathrm{O}}$ & $\overline{\text { BEF1 }}$ \\
\hline R5 & D1-1/O & \\
\hline R6 & 1/O & ACT3 \\
\hline$\overline{\mathrm{R} 7}$ & D2-I/O & \\
\hline$\overline{\mathrm{R} 8}$ & CSI - I/O & \\
\hline R9 & D4 - I/O & \\
\hline $\bar{R} 10$ & CSO - I/O & \\
\hline R11 & I/O & C7 \\
\hline $\bar{R} 12$ & $\mathrm{I} / \mathrm{O}$ & C3 \\
\hline R13 & 1/O & C1 \\
\hline$\overline{\mathrm{R} 14}$ & DONE - PG & \\
\hline $\bar{R} 15$ & RESET & \\
\hline R16 & I/O & READYo \\
\hline & & \\
\hline T1 & N.C. & \\
\hline T2 & N.C. & \\
\hline T3 & N.C. & \\
\hline T4 & I/O & BEF2 \\
\hline T5 & I/O & BEF3 \\
\hline T6 & $\mathrm{I} / \mathrm{O}$ & AFT3 \\
\hline T7 & I/O & AFT2 \\
\hline T8 & I/O & RELO \\
\hline T9 & $\mathrm{I} / \mathrm{O}$ & REL2 \\
\hline T10 & T/O & REL3 \\
\hline T11 & DS - I/O & \\
\hline T12 & $1 / 0$ & C4 \\
\hline T13 & $\mathrm{I} / \mathrm{O}$ & $\mathrm{C} 2$ \\
\hline T14 & XTAL1(OUT) - BCLKIN-I/O & \\
\hline T15 & N.C. & \\
\hline T16 & N.C. & \\
\hline
\end{tabular}




\section{TESTING THE DESIGN}

Once the design had been programmed, converted to LCA files and placed and routed, then downloaded to the CCM2 board, it then needed to be tested with the cube calculus operations mentioned in Chapter III. A test plan was drafted to systematically test the functionality of the design in the realized hardware model. This testing began with the combinational operations, progressing on to the complex combinational operations then finally testing the sequential operations. The order of this testing was specifically chosen and will be talked about in the respective sections that follow.

\section{Combinational Operations}

The combinational operations were selected to be tested first for several reasons. First, and most important, the combinational operations do not require the use of the control unit $(\mathrm{CU})$ to produce its resultant cube. Secondly, the combinational operations do not make use of the internal positional state machine (PSM) within each IT. The operation stays in the state BEFORE for the entire operation, thus eliminating the clocking of the PSM, and eliminating problems that might be caused by the globally controlling CU. Finally, by testing the combinational operations first, this allows the user to verify that the internal logic of the ILU is functioning properly, especially in the RELATION block of each IT.

Supercube. The supercube operation was initially tested using two multi-valued 16-bit input cubes A and B, allowing the entire 8-IT ILU to be occupied for the operation. Input cube $A=X^{12345} Y^{12}$ and cube $B=X^{34} Y^{3456}$. These cubes may be seen in Karnaugh map representation in Figure 39. 


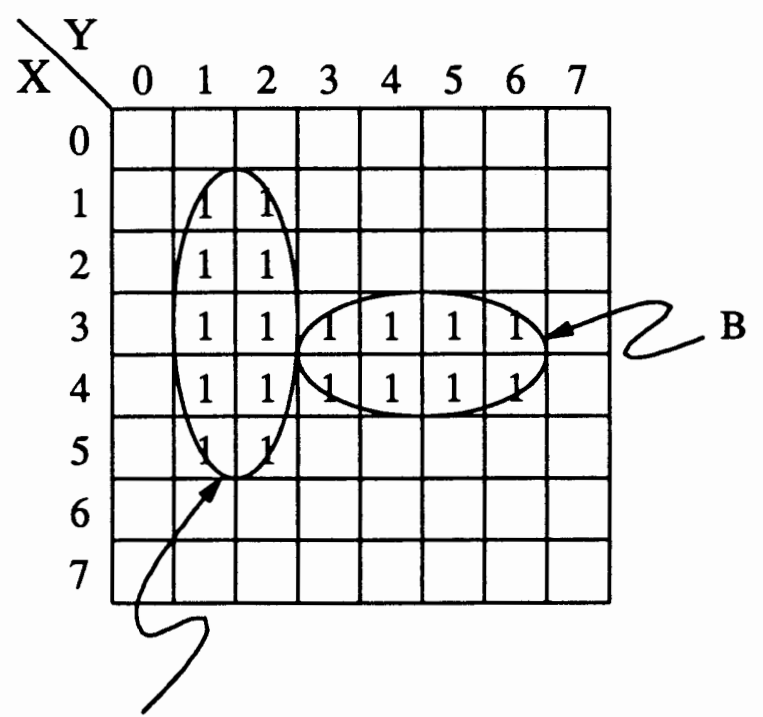

A

Figure 39. Supercube $-\mathrm{A} \cup \mathrm{B}=\mathrm{X}^{12345} \mathrm{Y}^{12} \cup \mathrm{X}^{34} \mathrm{Y}^{3456}$

The supercube operation is and AND-Type relation with a BEFORE relation of $A_{i} \cup B_{i}$. In the case of combinational operations, IR0 was set to "1" since the CU is not needed. To load the board with the proper values, the dip-switches were configured as follows: $A=0111110001100000 ; B=0001100000011110$; RIGHT_E $=00010001$;

$\mathrm{W}=00000000 ; \mathrm{BEF}=0111 ; \mathrm{REL}=1111 ; \mathrm{R}=10011000$. Upon applying power to the board and downloading the design from the Xinlix tools, the resultant cube lights produced the following values: 0111110001111110 . This resultant cube value is $\mathrm{X}^{12345} \mathrm{Y}^{123456}$, which is the correct result for this operation. Figure 40 shows the Karnaugh map representation of the resultant value for the supercube operation. 


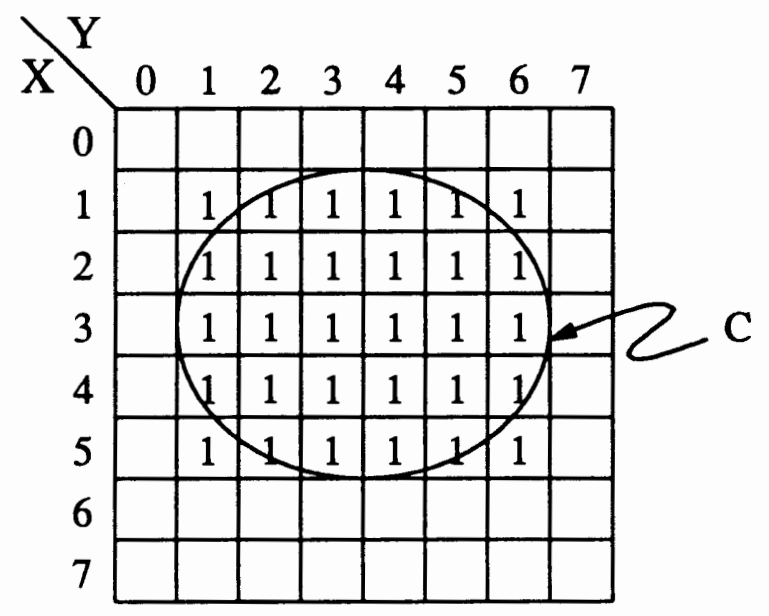

Figure 40. Supercube resultant cube $C=\mathrm{X}^{12345} \mathrm{Y}^{123456}$.

Intersection. Once the supercube operation was confirmed in its operation, the intersection operation was then tested using similar 16-bit input cubes. Input cube $A=$ $X^{23456} Y^{123}$ and $B=X^{45} Y^{2345}$. Figure 41 shows a Karnaugh map of the input cubes for the intersection operation. 


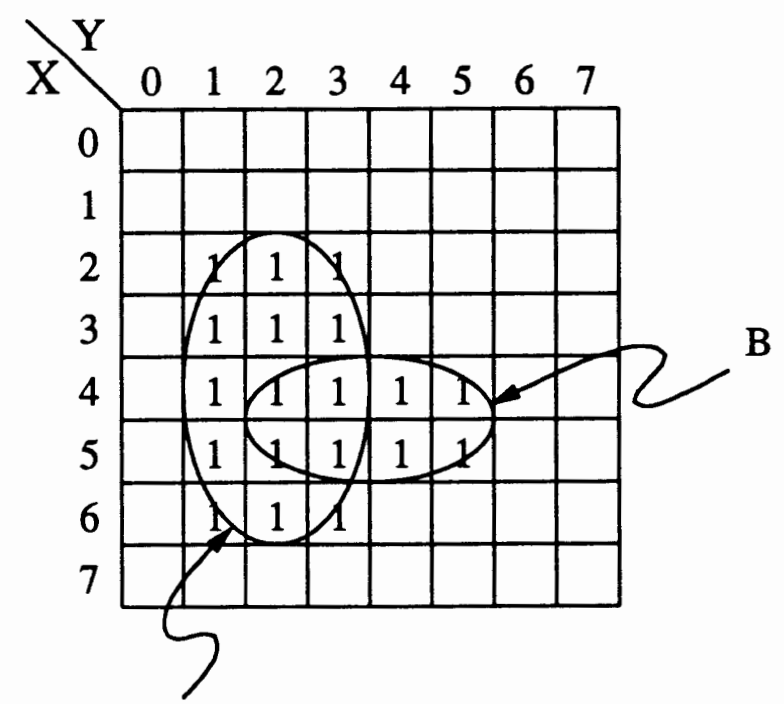

A

Figure 41. Intersection - $\mathrm{A} \cap \mathrm{B}=\mathrm{X}^{23456} \mathrm{Y}^{123} \cap \mathrm{X}^{45} \mathrm{Y}^{2345}$.

To load the board with the proper values, the dip-switches were configured as follows: $A=0011111001110000 ; B=0000110000111100$; RIGHT_E $=00010001 ; \mathrm{W}=$ $00000000 ; \mathrm{BEF}=0001 ; \mathrm{REL}=1111 ; \mathrm{IR}=10011000$. Upon applying power to the board and downloading the design, the resultant cube lights produced: 0000110000110000 . This is the correct value for the resultant cube $\mathrm{C}$ which has the value $\mathrm{X}^{45} \mathrm{Y}^{23}$. Figure 42 shows a Karnaugh map of the resultant cube $\mathrm{C}$. 


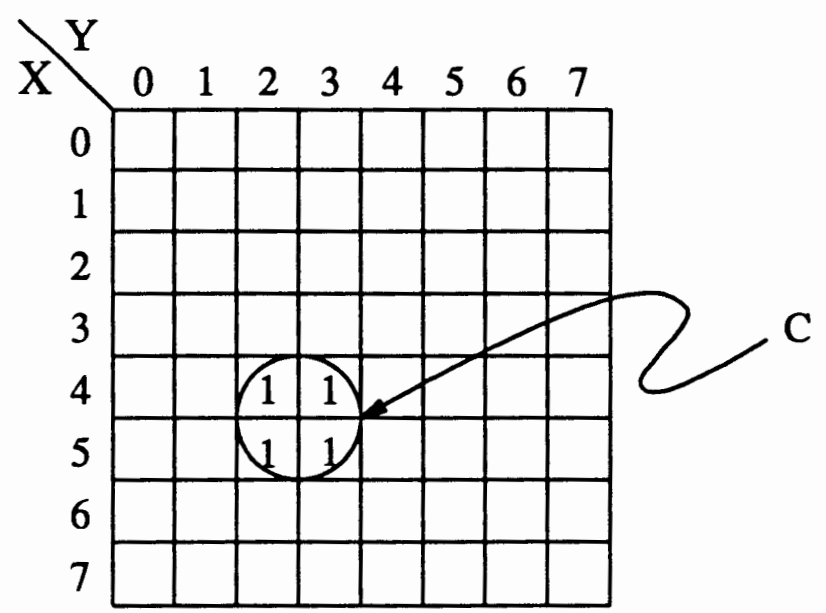

Figure 42. Intersection resultant cube $\mathrm{C}=\mathrm{X}^{45} \mathrm{Y}^{23}$.

Sequential Operations

Once the combinational operations had been proven in their operation within the ILU, the sequential operations were then tested. This class of operations uses the entire IT for the two bits that are to be operated upon, including the PSM which is not needed for the combinational operations. This means that for proper operation upon two bits of an input cube, proper timing must be achieved between the ILU and the CU since the clocking of the PSMs, upon which generates the proper values for each prime implicant of the resultant value, is controlled by the CU.

The $\mathrm{CU}$ is a scaled down version of the original design, since it also controlled the interface to the bus logic. Since the goal of this thesis is to realize only the ILU, the CU is only partially functioning. The following sections will show the examples that were tested on the design and present the values received as well as the values expected.

Crosslink. The crosslink operation was tested using two multi-valued 8-bit input cubes A and B, using only the first 4 ITs of the ILU to be used for the operation. This 
allowed testing of the Water register W. The input cubes for this operation may be seen in Figure 42. This allowed testing of the Water register W.

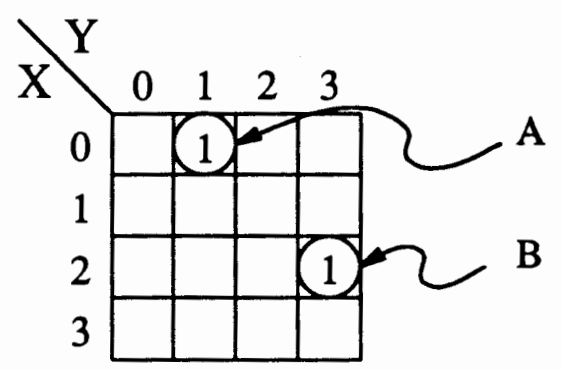

Figure 43. Crosslink $A \square B=X^{0} Y^{1} \square X^{2} Y^{3}$.

To load the board with the proper values, the dip-switches were configured as follows: $A=1000010000000000 ; B=0010000100000000$; RIGHT_E $=00010000 ; \mathrm{W}=$ 00001111; $\mathrm{BEF}=0011 ; \mathrm{ACT}=0111 ; \mathrm{AFT}=0101 ; \mathrm{REL}=1110 ; \mathrm{IR}=00011110$. Upon applying power to the board and downloading the design, the CU was reset to State 0 , then upon stepping through the states of the $\mathrm{CU}$, the resultant cube lights produced the first resultant cube $C_{1}=1010010000000000$. The $C U$ continued to step through to produce the second resultant cube $\mathrm{C}_{2}=001001010000000$. This is the correct value for the resultant cubes which have the respective values as shown in Figure 44.

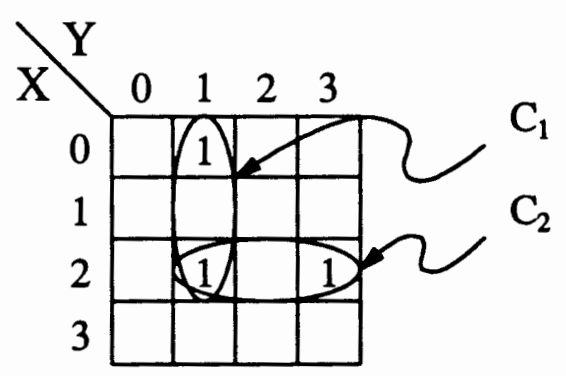

Figure 44. Resultant cubes $C_{1}=X^{02} Y^{1}$ and $C_{2}=X^{2} Y^{13}$. 
Symmetric Consensus. The symmetric consensus operation was tested using two multi-valued 16-bit input cubes A and B, occupying the entire 8 ITs of the ILU design. The input cubes may be seen in Karnaugh map representation in Figure 45.

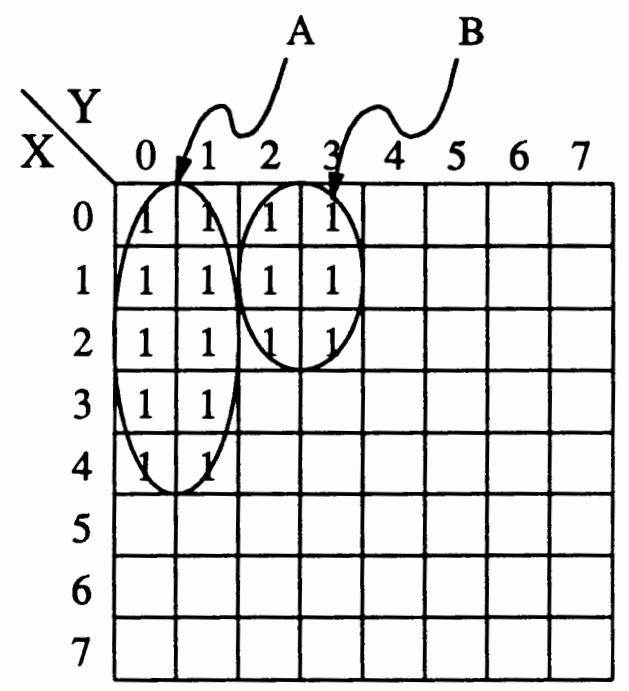

$\underline{\text { Figure 45. Symmetric consensus } A * s B=X^{01234} \mathrm{Y} 01 * s X^{012} Y^{23}}$.

To load the board with the proper values, the dip-switches were configured as follows: $A=1111100011000000 ; B=1110000000110000$; RIGHT_E $=00010001 ; \mathrm{W}=$ 00000000; $\mathrm{BEF}=0001 ; \mathrm{ACT}=0111 ; \mathrm{AFT}=0001 ; \mathrm{REL}=1111 ; \mathrm{R}=00011100$. Upon applying power to the board and downloading the design, the CU was reset to State 0, then upon stepping through the states of the $\mathrm{CU}$, the resultant cube lights produced the resultant cube $C_{1}=1110000011110000$. This is the correct value for the resultant cube which has the respective value as shown in Figure 46. 


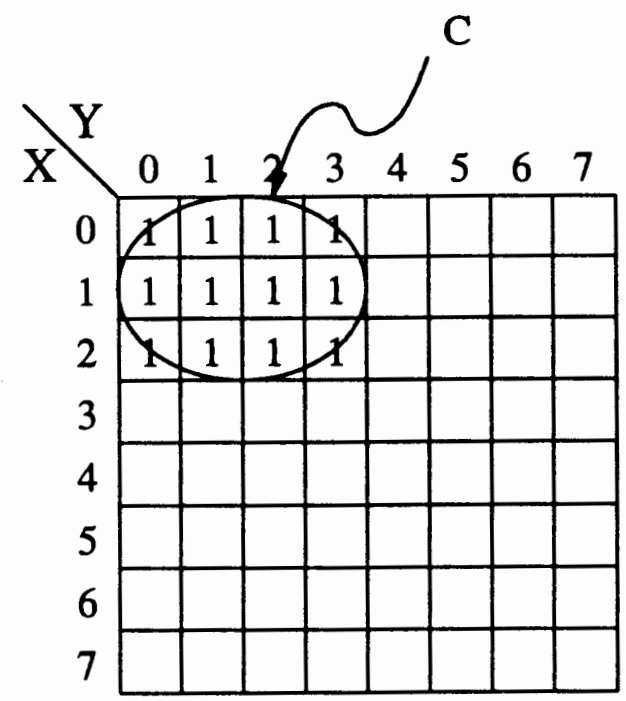

Figure 46. Resultant cube $\mathrm{C}=\mathrm{X}^{012} \mathrm{Y}^{0123}$.

Non-Disjoint Sharp. The nondisjoint sharp operation was tested using multi-valued input cubes occupying the entire 8 ITs of the ILU, through two multi-valued variables and one binary valued variable. Figure 47 shows the input cubes in Karnaugh map representation. 


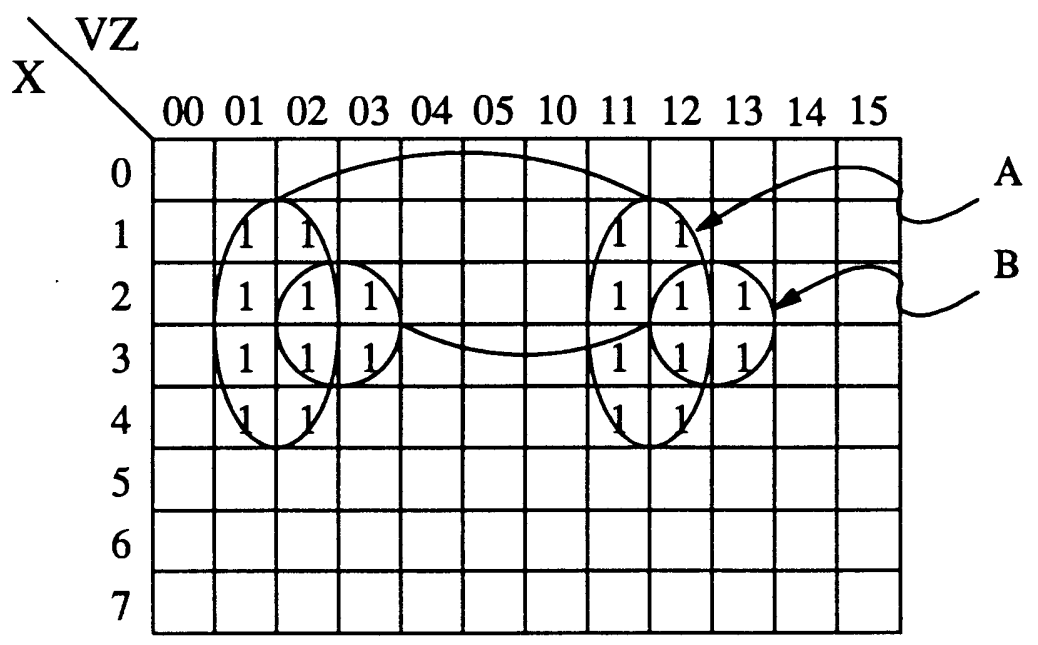

Figure 47. Sharp A \# B U ${ }^{12345} \mathrm{~V}^{01} \mathrm{Z}^{12} \# \mathrm{U}^{23} \mathrm{~V}^{01} \mathrm{Z}^{23}$.

To load the board with the proper values, the dip-switches were configured as follows: $A=0111100011011000 ; \mathrm{B}=0011000011001100$; RIGHT_E $=00011001 ; \mathrm{W}=$ $0000000 ; \mathrm{BEF}=0011 ; \mathrm{ACT}=0010 ; \mathrm{AFT}=0011 ; \mathrm{REL}=0010 ; \mathrm{AND} \_\mathrm{OR}=0 ; \mathrm{R}=$ 00000010. Upon applying power to the board and downloading the design, the CU was reset to State 0 , then upon stepping through the states of the $\mathrm{CU}$, the resultant cube lights produced the resultant cubes $C_{1}=0100100011011000$ and $C_{2}=0111100011010000$. These are the correct values for the resultant cubes which have the respective values as shown in Figure 48. 


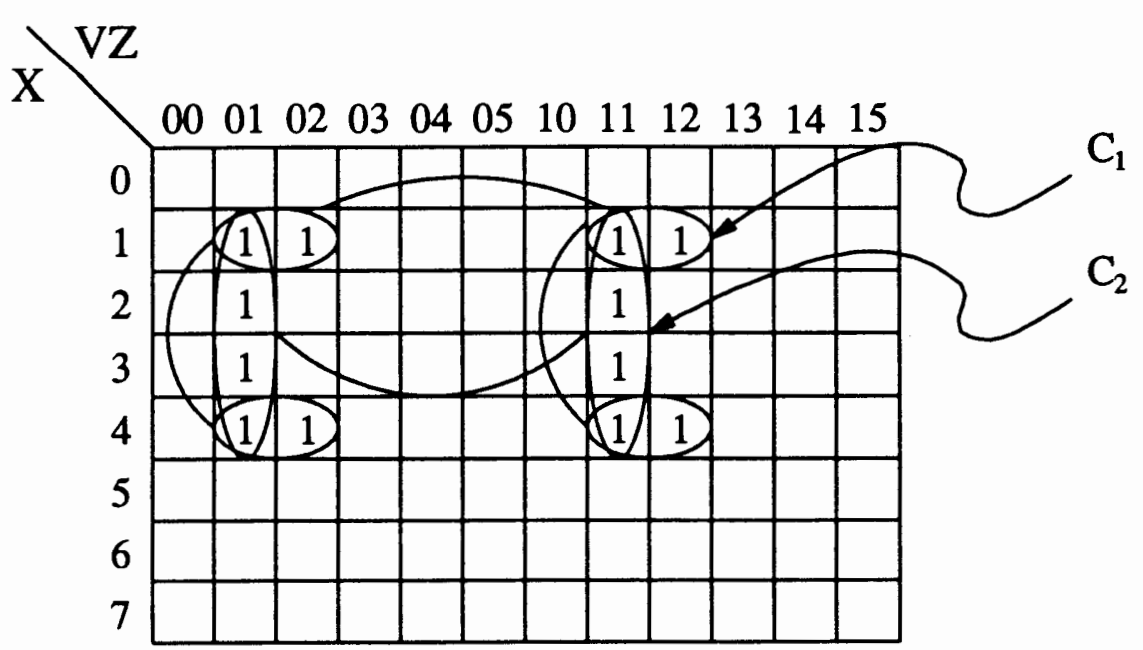

Figure 48. Non-disjoint resultant cubes $\mathrm{C}_{1}=\mathrm{U}^{14} \mathrm{~V}^{01} \mathrm{Z}^{12}$ and $\mathrm{C}_{2}=\mathrm{U}^{1234} \mathrm{~V}^{01} \mathrm{Z}^{1}$.

Disjoint Sharp. The disjoint sharp operation was tested using the identical multivalued input cubes as in the nondisjoint sharp operation. The only difference here is in the relation of AFTER. To load the board with the proper values, the dip-switches were configured as follows: $\mathrm{A}=0111100011011000 ; \mathrm{B}=00110000011001100$; RIGHT_E $=$ $00011001 ; \mathrm{W}=00000000 ; \mathrm{BEF}=0011 ; \mathrm{ACT}=0010 ; \mathrm{AFT}=0001 ; \mathrm{REL}=0010 ;$ AND_OR $=0 ; \mathrm{IR}=00000010$. Upon applying power to the board and downloading the design, the CU was reset to State 0 , then upon stepping through the states of the $\mathrm{CU}$, the resultant cube lights produced the resultant cubes $C_{1}=0100100011011000$ and $C_{2}=$ 0011000011010000 . These are the correct values for the resultant cubes which have the respective values as shown in Figure 49. 


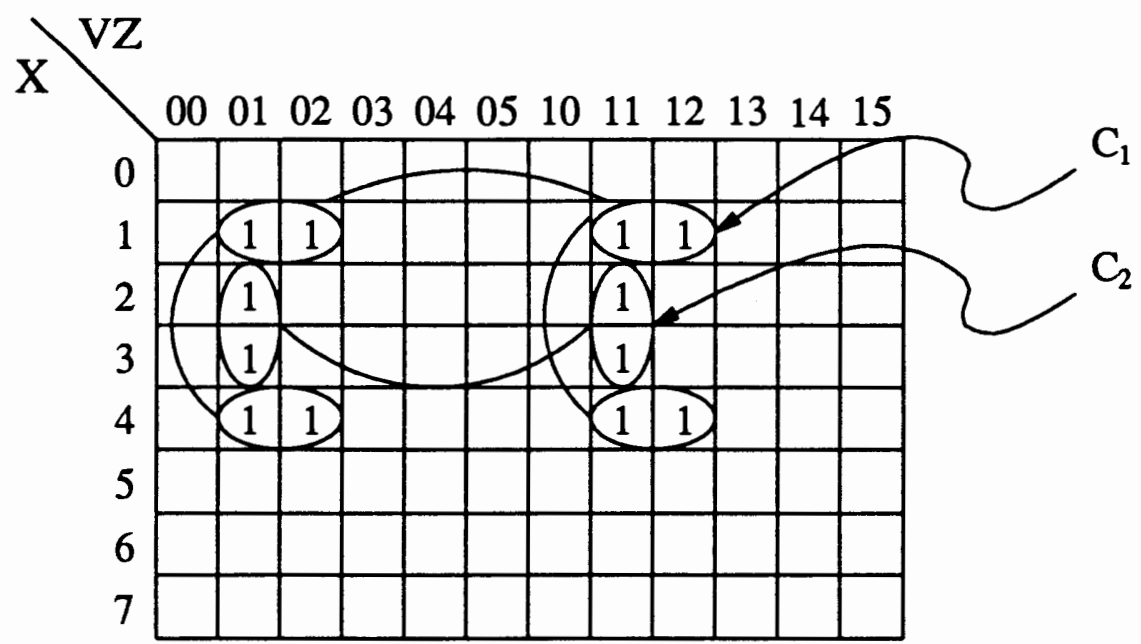

Figure 49. Disjoint resultant cubes $\mathrm{C}_{1}=\mathrm{U}^{14} \mathrm{~V}^{01} \mathrm{Z}^{12}$ and $\mathrm{C}_{2}=\mathrm{U}^{23} \mathrm{~V}^{01} \mathrm{Z}^{1}$.

These operations are a mere beginning to introducing the functionality of the ILU of the CCM2. The selected examples give a comprehensive coverage of the tests needed to be run on the ILU to verify its performance and to introduce a new user to the operability of the design. Resolutions of the problems encountered, solutions to these problems and what lies ahead for future CCM designs is covered in the conclusions in Chapter VII. 


\section{CHAPTER VI}

\section{DESIGN EVALUATION AND COMPARISON}

Now that the ILU has been verified in its operation, a proper timing analysis must be done to complete the evaluation of the CCM2 hardware. By analyzing the CCM2's timing characteristics, both the internal delays of the logic devices and the amount of time it takes to process data received at the inputs and produce the results at the outputs must be taken into account. Since only the ILU has been investigated in this thesis, all external delays to the hardware will not be considered.

\section{TIMING ANALYSIS}

When determining the internal timing of the ILU, there are three types of delays that must be investigated: vertical signals received from the host computer at the inputs and leaving the ILUs outputs; horizontal signals received from the CU at the inputs of the first IT and returned from the outputs of the last IT back to the $\mathrm{CU}$; and lastly the delays incurred from executing sequential and complex combinational operations upon the input cubes.

The vertical signals include input, output, prerelation, relation and instruction register signals. The delay of concern here is the time it takes to compute a combinational operation once the input cube values have been received, then passed to the outputs of the device. For simple combinational operations such as intersection and supercube, the $\mathrm{CU}$ is not needed, so the time involved in calculating the output cubes is determined by the delay of the signals through the RELATION subsection of each IT. 
With the most recent placement and routing of the CCM2 design, it was found that the greatest delay was in the first IT of each device. The delay of 145.8 nanoseconds was the greatest delay found, while a delay of 89.2 nanoseconds was the smallest delay found in the last IT of each Xilinx device. All ITs within the ILU have the identical design, so the reason for the 56.6 nanoseconds difference in delays is caused by the placement and routing of the design. Thus, these delays are likely to vary each time the automatic placement and routing routine is run.

The horizontal signals include NEXT, READY, CLEAR, PRIME and REQUEST, and are the signals used in the generation of cubes from sequential and complex combinational operations. All of these signals are received by or returned to the $\mathrm{CU}$, however, only the NEXT and READY signals will be considered here, since they are critical for the generation of output cubes. CLEAR, PRIME and REQUEST are received at each IT at approximately the same time and do not get passed to adjacent cells during operation. The NEXT and READY signals are used to coordinate the states at which specific ITs of the ILU should be for the generation of multiple output cubes. These signals ripple through the chain of ITs under the control of the CU. Obviously the delay is dependent upon the size of the input cubes and the number of output cubes produced, so the delay times are based upon all ITs being used, and the ILU producing a single resultant cube. The signal NEXT has a delay of 353.4 nanoseconds while READY has a delay of 219.4 nanoseconds. It will be shown in the following section that these two signals are the limiting factor in the speed of my design. The attached timing sheets found in Appendix B, show the delays of the two signals found for a single device. It can be seen that a major source of delay is from the signal entering and exiting the device to the $\mathrm{I} / \mathrm{O}$ pins. Once the design is fit into a single programmable device, these delays will decrease significantly since the signals must currently pass through two devices. 
To justify the need for special hardware to accelerate the processing of logic functions, we must first evaluate existing logic minimization programs and determine which software routines might benefit from this hardware. Many logic minimization software tools such as ESPRESSO [4][45][46], MINI [27], and MIS II [5][34] may benefit from the addition of the CCM2. In ESPRESSO, there is no direct relation between any one measure of problem size (inputs, outputs, literals, terms, and so forth) and the computing time. If a routine is detected that would greatly benefit from the addition of the CCM2, it may then justify the additional cost depending upon the frequency that the routine is called during typical operation.

It was found that one essential algorithm used in ESPRESSO-II could have its processing time significantly reduced with the addition of the CCM2. The COMPLEMENT procedure is executed exactly once during typical operation, and consumes $14 \%$ of the total CPU time on average [4]. It is second in time consumption to the EXPAND procedure which on average requires $29 \%$ of the CPU time. The EXPAND procedure uses the results of the complement to quickly determine primes covering a given cube and to help guide which of the primes will be chosen to produce the best cover. One might chose to eliminate the COMPLEMENT algorithm, however, the initial cost of the procedure is offset by the increase in computing time needed by EXPAND. In addition, the quality of the cover obtained after each expand step would be reduced.

On the CCM2, a combinational operation such as complement [8][26][37][48] may be executed in six clock cycles of the $\mathrm{CU}$ for a single resulting output cube. Assuming an Intel 80486 or equivalent clone processor with a system clock of $33 \mathrm{MHz}$, giving a period of 30.30 nanoseconds per clock cycle, the result of a single cube output from the complement of an input function may be calculated in 181.80 nanoseconds. This may be seen in Figure 21 on page 48, showing the single resultant cube being calculated after 6 
clock cycles. For multiple resultant cubes, the first cube is produced after 6 clock cycles, then the subsequent output values are produced after every additional three clock pulses, until the NEXT signal has been received. Thus, the complement of a function producing two resultant cubes will take 272.70 nanoseconds, three resultant cubes will take 363.60 nanoseconds, and so on. These figures are not entirely true since the calculation cannot be written to the data bus until the NEXT signal is received. This takes 353.4 nanoseconds to be received, then an additional 30.30 nanoseconds is needed to reset the CU back to its initial state. This combines for a total of 383.7 nanoseconds for one or two resultant cubes. If three resultant cubes were produced, it would take 393.30 nanoseconds. From this data, it can be seen that for cube calculus operations producing multiple cubes, there is a great speedup benefit since only 3 clock cycles are needed for each additional resultant cube after the first one has been generated.

Assuming that the COMPLEMENT function was sped up by one thousand times, this still does not justify the addition of special hardware since we are essentially speeding up 14 percent of the entire processing operation. The remaining 86 percent will be calculated in the same amount of time as before. However, many software tools and special hardware accelerators are designed to handle specific minimization problems and operations, and this is where we feel the CCM2 will find its niche. Two such possible problems presented below are satisfiability and tautology [37][38]. Both of these problems may take advantage of the complement and sharp (\#) operations available on the CCM2.

\section{$\underline{\text { Satisfiability }}$}

The satisfiability problem asks the question "for what values of arguments is a particular formula satisfied". Basically, "are there any cells or groups of cells of a Karnaugh map of function $f$ that equal 1." The satisfiability problem may be reduced to 
the tautology problem and vice-versa through the complementation operation.

If the function $f(a, b, c)=1$, this implies that $1 \# f(a, b, c)=\varnothing$. This may also be written as $1 \# \mathrm{f}=1 \cdot \overline{\mathrm{f}}=\overline{\mathrm{f}}$, which is the complement of the function $\mathrm{f}$. Figure 50 shows the function $f=X^{56} Y^{012345678}$ for which the complement of the function equals $1 \cdot \bar{f}=\bar{f}$.

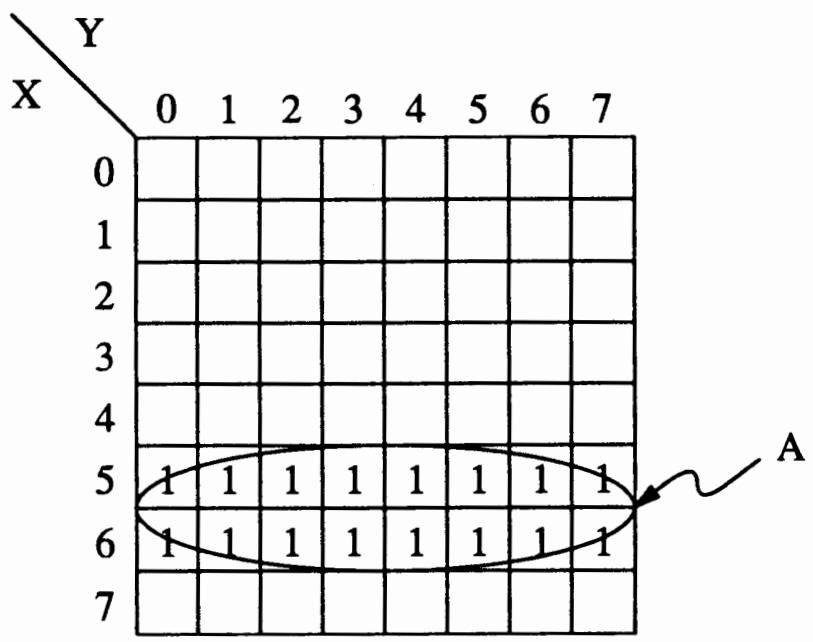

Figure 50. Input cube $A=X^{56} Y^{01234567}$

The complement of the input cube $A$ is as follows:

$$
\bar{A}=\left\{X_{1}{ }^{1} \cdots X_{i-1}{ }^{1} X_{i}{ }^{\sim A_{i}} X_{i+1}{ }^{1} \cdots X_{N}{ }^{1} \text { I for such } i=1, \ldots, N \text {, that } A_{j} \neq 1\right\}
$$

The resultant cube of the complement function on input cube $A$ is equal to $\mathrm{X}^{012347} \mathrm{Y}^{01234567}$ and may be seen in Figure 51. 


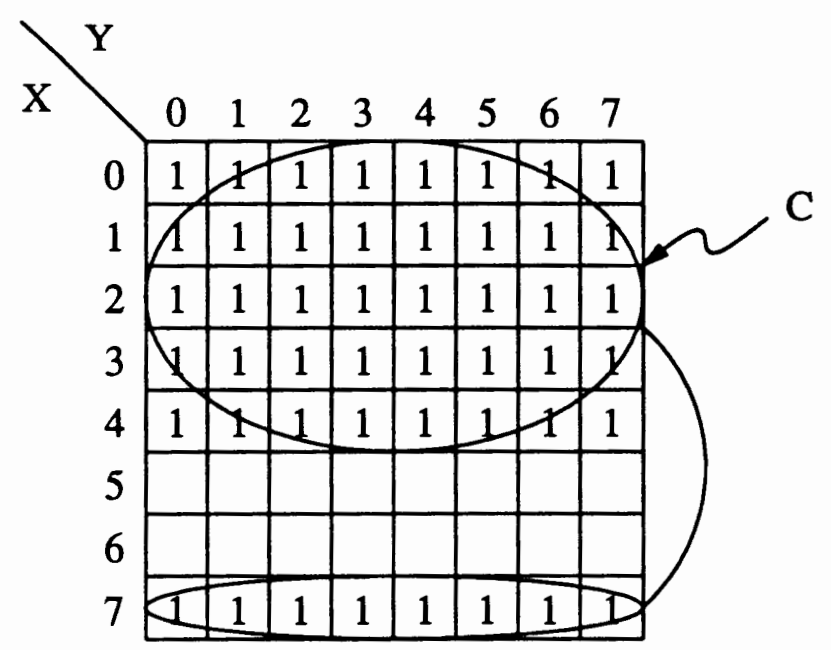

Figure 51. Resultant cube $\mathrm{C}=\overline{\mathrm{f}}=\mathrm{X}^{012347} \mathrm{Y}^{01234567}$

\section{Tautology}

The tautology problem is the verification of a logical formula to see if it is always true such as verifying whether $a b+a=a$. If the function $f(a, b, c)=1$, this implies that $1 \#$ $f(a, b, c)=\varnothing$. If $f=\bar{a} \bar{c}+\bar{a} \bar{b} c+b c+a b \bar{c}+a \bar{b}$, then $1 \# f$ must equal $\varnothing$. The sharp of two cubes $\mathrm{A}$ and $\mathrm{B}$ denoted $\mathrm{A} \# \mathrm{~B}$, is a set of all of the largest cubes included in $\mathrm{A}$ and not included in $B$. Given the function $f(a, b, c)=\bar{a} \bar{c}+\bar{a} \bar{b} c+b c+a b \bar{c}+a \bar{b}$, we can see in the Karnaugh map in Figure 52 that the function equals one although that is not apparent to us by just looking at the expression of the function. 


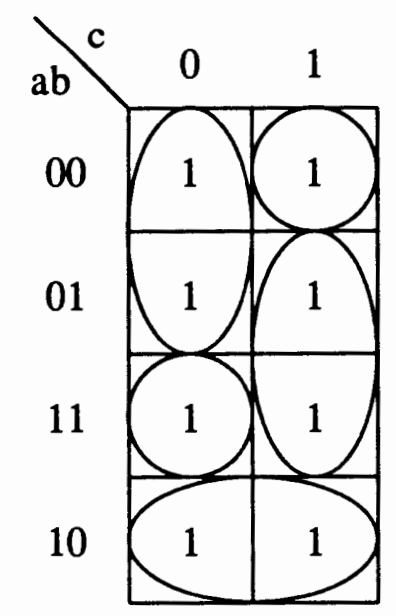

Figure 52. $f(a, b, c)=\bar{a} \bar{c}+\bar{a} \bar{b} c+b c+a b \bar{c}+a \bar{b}$

In order to verify if the function is equal to one, our methodology would be to sharp a cube of the input function from the function $f=1$, then take the resultant function $f^{\prime}$ and sharp the next cube of the original input function from it, iterate for all cubes of the input function and check if the final result is empty. This method may be seen in Figure 53. 

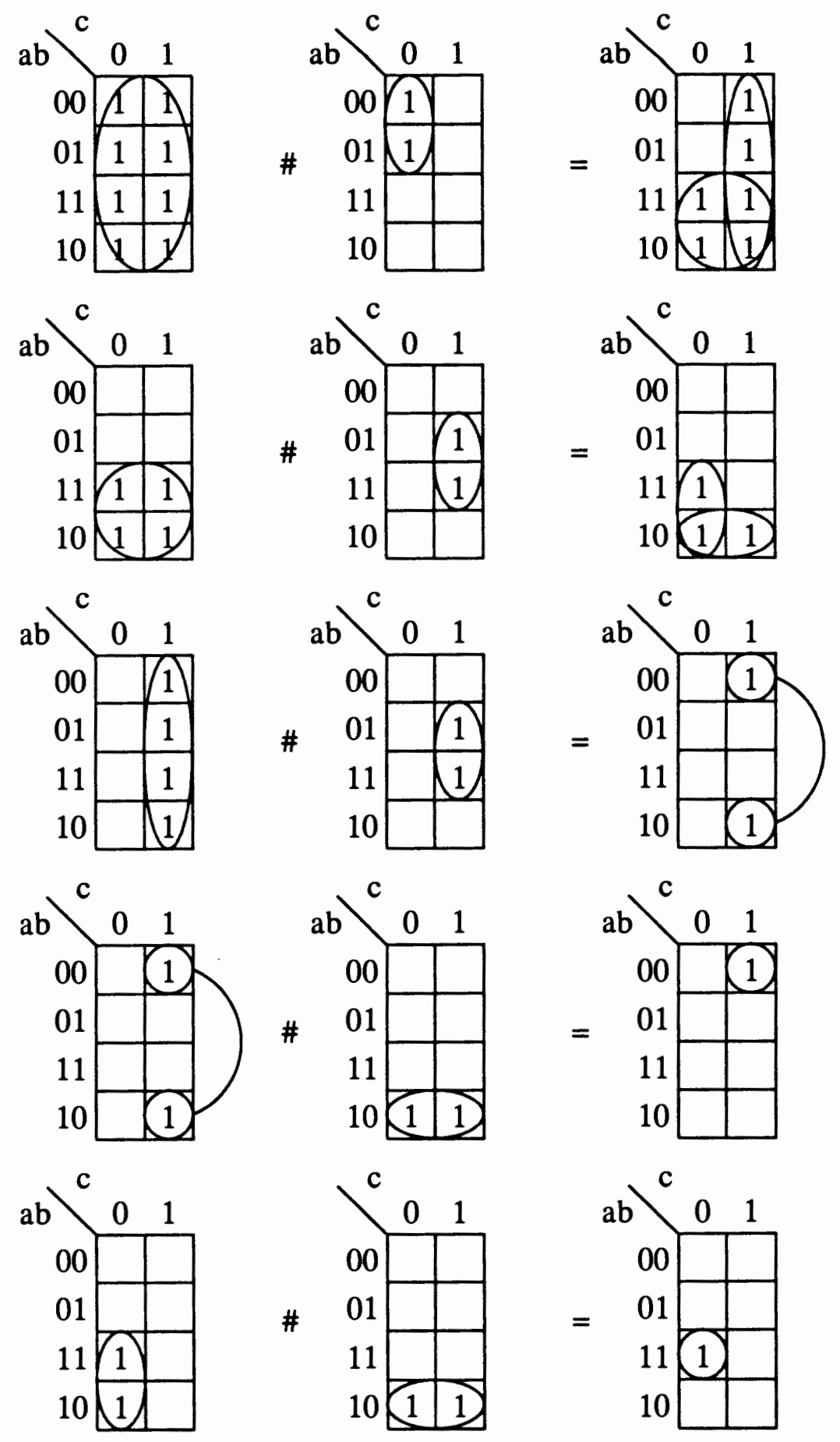

Figure 53. TautologyVerification. 

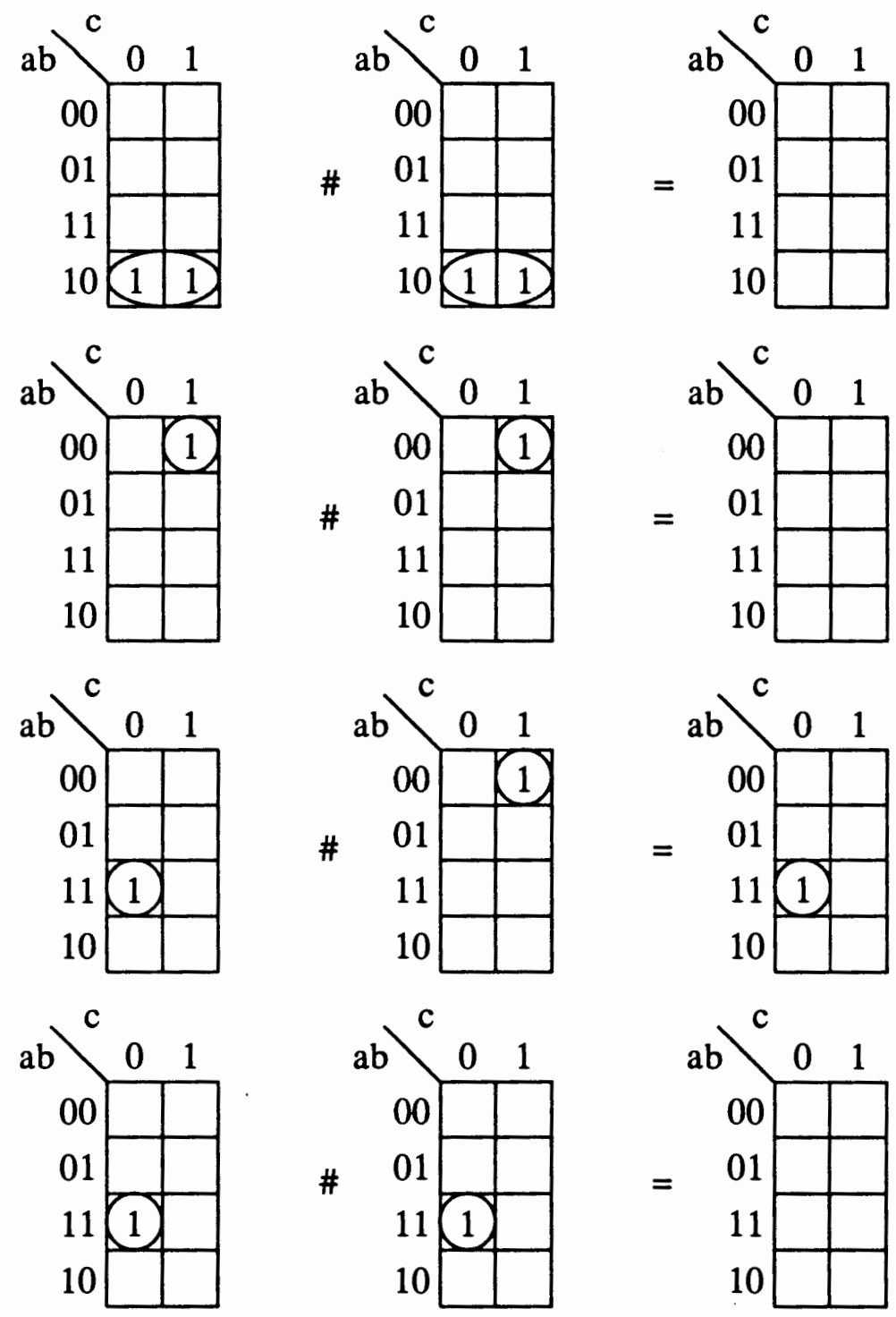

Figure 53(Continued). TautologyVerification.

Observing the methodology that is used in Figure 53 to prove that our input function $F$ is equal to one, the first input cube $\bar{a} \bar{c}$, is sharped from a function equal to "1". The two resultant cubes from this operation are stored, then they each sharp the second cube of the input function, storing the resultant cubes from each nondisjoint sharp operation. This process is repeated until there are no further input cubes left. The final result is checked, 
and if the final resultant cube is equal to zero, then we have indeed verified that our input function of $f(a, b, c)=\bar{a} \bar{c}+\bar{a} \bar{b} c+b c+a b \bar{c}+a \bar{b}$ equals 1 . From this example of tautology, I have written a small program that executes the tautology algorithm based on these principles.

This program was created in order to determine the speedup of such a problem using the CCM2 hardware instead of using a software manipulation. Located in Appendix D, is the $\mathrm{C}$ code program that simulates the above tautology example. The code was developed on an Intel $486 \mathrm{DX}-33$ platform, using a Microsoft $\mathrm{C}$ version 6.0 compiler. The granularity of the time reported back from a PC is accurate to approximately $1 / 18$ th of a second, so the program was placed in a loop that iterated the tautology problem one million times in order to obtain a reasonable time measurement. The program was able to compute the tautology example in approximately 33.56 microseconds. Using the times that were calculated earlier for sequential operations to be processed in the CCM2's ILU, I was then able to determine the processing time for the same tautology example.

It was found that the nondisjoint sharp operation was performed ten times for this particular example, with each operation producing anywhere from zero to two resultant cubes per sharp operation. This time was determined to take a total of 2.30 microseconds. Comparing the software and hardware times shows that the ILU of the CCM2 is able to accelerate the operation by approximately 13.59 times. This speedup is based only upon the ILU functionality, and does not reflect external signals to the Xilinx devices such as the host and bus control operations. These times will change significantly when the bus control features are added by future classes. Once the final design, placement and routing changes are made, the ILU delays will likely decline. Additionally, the LCA design may be ported to an ASIC device, and the programmable connections will be hardwired, dropping delay times considerably. 
After observing the differences in the processing time between the hardware and software implementations of the three variable tautology example, I decided to try some examples of other tautology problems with varying numbers of input variables. TABLE XI shows the variation of problems attempted. There is a definite increase in the speedup ratio between the hardware and software, however, the smaller problems had two input cube features changing, thus I was unable to determine what the true cause of the speedup was. I then tried some larger input functions, holding the number of input cubes within each function to be constant, thus making the additional variable a don't-care value in the input function. By doing this, I was able to see that the CCM2 was able to process input functions of 4,5 , and 6 variables at the same amount of time. The software simulation, however, steadily increased in time with the addition of extra variables. This is due to the software's need to iterate through an another loop for each additional variable that is presented in the input function. Figure 54 shows the speedup comparison of multivariable tautology functions that were tested.

\section{TABLE XI}

\section{DIFFERING VARIABLE SIZE SHARP COMPARISON}

\begin{tabular}{|l|c|c|c|c|c|}
\hline Number of Variables & 2 & 3 & 4 & 5 & 6 \\
\hline Number of Input Cubes & 3 & 5 & 7 & 7 & 7 \\
\hline \hline Software Sharp Simulation ( $\mu \mathrm{s})$ & 12.53 & 33.56 & 135.67 & 162.58 & 182.63 \\
\hline CCM2 Sharp Operation $(\mu \mathrm{s})$ & 1.15 & 2.30 & 7.32 & 7.32 & 7.32 \\
\hline \hline Hardware Speedup & 10.90 & 14.59 & 18.53 & 22.21 & 24.95 \\
\hline
\end{tabular}

I then held the number of variables constant at three, and constructed 10 different covers, ranging from one to ten input cubes. TABLE XII shows the results obtained from this trial. As you can see, the software ramps up at a linear increase in processing time as 
the variable count rises. The graphical representation of this may be seen if Figure 55. A large hit in processing time is taken on the seven input cube example. This may be attributed to the manner in which the algorithm processes the tautology example. If the function were ordered in a slightly different manner, less resultant cubes would likely result, since the generation of multiple resultant cubes adds an additional loop of computation. The CCM2 also increases linearly, however, it tends to level off. This is attributed to the fact that the tautology has likely been proven at this point. With a three variable function, the cover may be made with eight of the smallest possible input cubes.

\section{TABLE XII}

\section{3-VARIABLE SHARP COMPARISON}

\begin{tabular}{|l|c|c|c|c|c|}
\hline Number of Input Cubes & 1 & 2 & 3 & 4 & 5 \\
\hline Software Sharp Simulation $(\mu \mathrm{s})$ & 5.33 & 10.55 & 14.86 & 19.50 & 33.56 \\
\hline CCM2 Sharp Operation $(\mu \mathrm{s})$ & 0.38 & 0.77 & 1.15 & 1.53 & 2.30 \\
\hline Hardware Speedup & 14.03 & 13.70 & 12.92 & 12.75 & 14.59 \\
\hline \hline Number of Input Cubes & 6 & 7 & 8 & 9 & 10 \\
\hline Software Sharp Simulation $(\mu \mathrm{s})$ & 30.37 & 128.18 & 85.90 & 45.97 & 39.88 \\
\hline CCM2 Sharp Operation $(\mu \mathrm{s})$ & 2.30 & 5.76 & 6.94 & 5.76 & 3.07 \\
\hline Hardware Speedup & 13.20 & 18.47 & 14.91 & 14.97 & 17.34 \\
\hline
\end{tabular}

It can be noted from the internal delays of the Xilinx devices, that large functions with many variables will be what fully utilizes the functionality of the CCM2. Small functions such as the three variable tautology example presented in this thesis, don't necessarily utilize the parallel architecture of the ILU. These small functions are crippled by the delays inflicted by the horizontal signals that are used to control the output of the resultant cubes. The CCM2 must also target logic minimization problems that are quite repetitive in their structure such as the tautology example, or applications that are extremely cube calculus intensive. To simply replace a small percentage of cube calculus 
operations within an existing logic minimization package would not be worth the added expense. 


\section{Differing Variable Size Sharp Comparison}

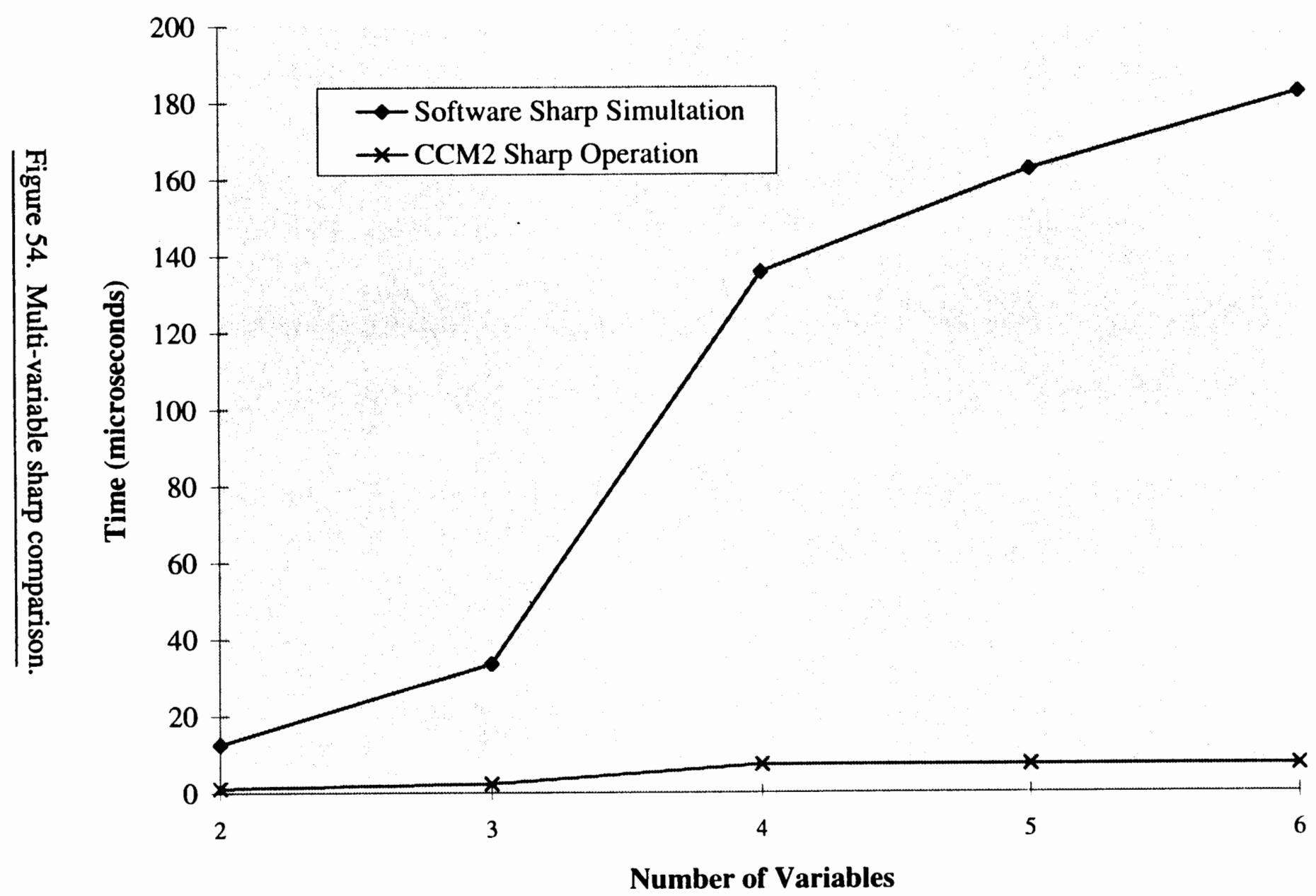


3-Variable Sharp Comparison

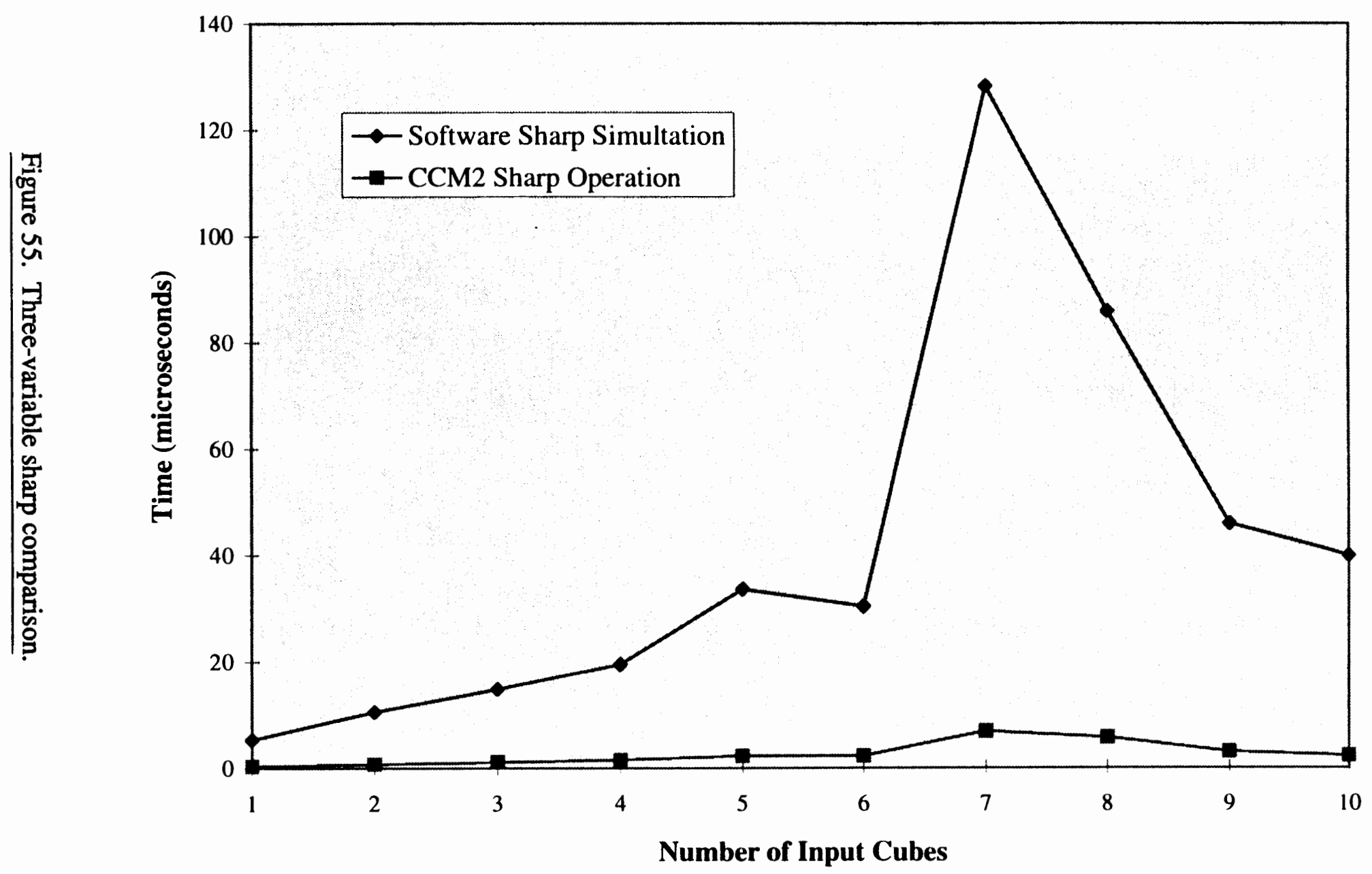




\section{BDD COMPARISON}

An alternative method to Cube Calculus in the minimization of logical functions is through the use of Binary Decision Diagrams (BDDs) [1][6][8][20][34]. BDDs are a data structure used to represent boolean functions and an associated set of manipulation algorithms. BDDs support many common logical operations including and, or, xor, smoothing, consensus, containment, tautology and satisfiability. Functions are represented by directed, acyclic graphs commonly referred to as DAGs. The resulting graph is a concise description of a logical function telling the user how to determine the output value of the function by examining the values at the inputs. Considering the function $\mathrm{F}=\mathrm{A}+\overline{\mathrm{BC}}$, the resulting $\mathrm{BDD}$ is created in Figure 56.

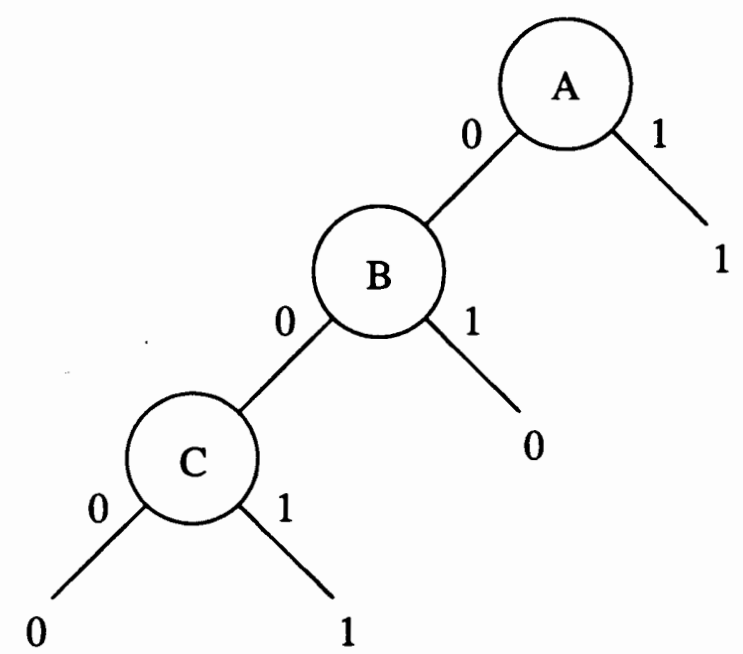

Figure 56. Function $\mathrm{F}=\mathrm{A}+\overline{\mathrm{B}} \mathrm{C}$ in $\mathrm{BDD}$ representation.

Each node in the diagram refers to a single variable from the function $F$, with each branch leading in the direction of the value of the variable. A node may have the value of 0 or 1 . With $n$ variables, there will be an initial $2^{\mathrm{n}}-1$ nodes in such a diagram. Reducing this number has been the focus of research in BDD implementations over the 
past decade.

Nodes may be easily removed by observing the structure of the diagram and removing ones that lead to identical output values regardless of the variable value. In addition, two separate nodes may both lead to the same output value and are capable of being merged. These two methodologies provide simplification to the diagram, however, with the exponential increase in the number of nodes, large logic functions require significant amounts of processing time.

The size of the BDD representation for a given function is extremely sensitive to the selection of variable order. The problem of finding the optimal variable ordering is NPcomplete, with all known exact methods being based upon exhaustive searches. As a result of this ordering dependence, poor orderings can limit the use of BDDs in complex problems due to finite memory resources. In addition, optimal CPU times are achieved by good variable orderings since the time to perform a BDD operation is proportional to the size of the BDD. Thus, considerable research is focusing upon heuristics for finding good orderings.

Ordered BDDs (OBDDs) are currently being explored for use in synthesizing and analyzing combinational and sequential circuits, with the emphasis of their power being directed towards design verification [7][16][36]. By developing clever representations and efficient manipulation algorithms, it is possible to avoid the time consuming exponential computations of simple BDD structures. Reduced Ordered BDDs (ROBDDs) have been used in different stages of logic design due to their representative form of Boolean functions and canonical structure [3][32][35]. ROBDDs have been proven in signal and fault detection applications since they may be constructed to represent the detection function. Modified BDDs (MBDDs) are simply ROBDDs with a third terminal used as a don't-care variable [29]. The method is based on the replacement 
of the don't-care terminal by other subgraphs of the MBDD, thus leading to the reduction of the graphs size. This technique may be used in FPGA synthesis to simplify circuit subfunctions before mapping the design to the device.

These methodologies are all based upon heuristics which reduce the number of possible input variables, which directly effect the size of the graph structure. These heuristic techniques use partial simulations which allow the possibility that comparisons between two functions may not be detected. Additionally, these algorithms may require large amounts of memory for their storage, increasing cost and affecting the speed of computation. For large circuits, this is infeasible since the trade-off between speed and cost is so high. However, it has been determined that BDDs give much better results than with cube calculus.

The BDD structures have just recently gained researchers' attention over the last decade, and due to the before mentioned problems, not all logic designers have moved away from using cube calculus. At this time, it is not known by the author, the extent of ROBDD or MBDD structures that have been realized in hardware. Standard BDDs' have a simplistic structure which may be easily realized using 2-to-1 multiplexors. With this ability, recent research has focused on multiplexor-based FPGA synthesis. FPGA architectures such as ACTEL have configurable memory cells that are multiplexor-based, allowing easy mapping of Boolean functions of the BDD form. Due to advances in multiplexor-based programmable logic devices, mapping BDD designs to programmable resources will be quite convenient.

To do a proper comparison between the CCM2 design and a BDD logic minimization package, I had to compare existing packages using the two methodologies. Jacobi and Trullemans [29], present a logic minimization method using MBD's that they determined to perform at a 35 percent efficiency over ESPRESSO [4][45][46]. This 
efficiency is measured in the size of the function after the minimization process. From our data in TABLE XII, we achieve a speedup of over 14 times that of the software representation. If I make the assumption that my particular code is half as optimized as that of the sharp operation in ESPRESSO, I would be making the statement that the CCM2 is seven to eight times faster than ESPRESSO for the sharp operation. The efficiency in terms of minimization would be equal since they would both use the sharp operation to perform the optimization. I can then make the statement that the CCM2 should be able to out-perform the MBD optimization under these assumptions.

If these two methods were available in an off-the-shelf fashion to perform the same function for the same price, the CCM 2 would provide the best performance for the price. The BDD implementation might provide better performance in some instances such as the case seen in Figure 55, where the input cube ordering has a direct effect on the number of resultant cubes produced and the processing time needed. This appears to happen approximately 20 percent of time for the examples I have run.

Due to the programmable resources available, and the progress already achieved in the CCM2 design, cube calculus remained as the methodology of choice for the design. Future research should consider building a BDD-based accelerator board, or the possibility of a combined cube calculus and BDD board. With a universal hardware implementation such as this, logic minimization programs will be able to take advantage of both methodologies, adding flexibility and processing power to many applications. The difficulty of fitting the BDD structure to hardware, is that BDD's become quite complex for large input functions and are not as easily scaled as our CCM2 processor. BDD's have proven to be very suitable for software implementations, since the complex scalability that is needed may be easily achieved. 


\section{CHAPTER VII}

\section{CONCLUSIONS AND FUTURE WORK}

\section{CONCLUSIONS}

The purpose of the Cube Calculus Machine II (CCM2) is to execute logic operations in high-level formal systems in a real-time fashion. The CCM2 targets applications in logic optimization, logic synthesis, pattern recognition and image processing. It may be implemented as a coprocessor to a host computer, or developed as an application-specific add-in card, much like existing hardware accelerator cards.

Presented in this thesis has been the Iterative Logic Unit (ILU) of the CCM2. A 16-bit ILU, consisting of 8 iterative cells (ITs) has been realized using commercial, offthe-shelf Field Programmable Gate Arrays (FPGAs). The ILU is quite regular in its design, allowing equal portions (4 ITs) of the design to be divided between the two Xilinx Logic Cell Arrays (LCAs). FPGAs allow an ideal environment for university projects due to their off-the-shelf availability, PC-based design tools, and time-to-market capabilities. Design modifications may be made in hours versus weeks with the same design being realized in VLSI. Also, a single device may be used across multiple design applications consuming only minutes changing from one application to another.

My contributions to the CCM2 project are outlined below in the manner in which the project pieces were completed:

- Participated in the design and simulation of the CCM2 as a member of a group in Dr. Perkowski's EE510 Computer Architecture for Robotics and Artificial Intelligence courses during the 1991-92 school year. 
- Developed an application of the CCM2 for image processing using a modified Hough transform, which is not covered in this thesis.

Updated, improved and added understanding to previous descriptions and explanations of the CCM2 architecture and the cube calculus operations upon which the machine's data path is based, into a comprehensive and systematic teaching tool.

- Prepared a tutorial for classes to use to learn the design flow necessary for using the FutureNet DASH and Xilinx XACT development tools. This tutorial is presently being used by students of Dr. Perkowski for developing designs of other student projects.

Converted and improved the logic description of the ILU from previous literature and simulation models into a hierarchical schematic representation.

- Designed the FutureNet DASH schematic files of an IT cell that was mirrored and connected to additional IT cells to create the realization of the ILU schematic file.

Converted the schematic design to the Xilinx LCA files necessary for programming the FPGA devices.

- Constructed a demonstration board for the downloading and testing of the ILU design.

- Downloaded and tested the ILU design with the constructed board, providing multiple examples to aid future classes in the understanding of the operation of the CCM2. This was the first hardware realization of the ILU and will be used as a foundation for future expansions and improvements in the CCM design.

- Wrote a $\mathrm{C}$ code program simulating the sequential operation of nondisjoint 
sharp. This program was used to do a speedup comparison of the CCM2 over a typical software representation.

\section{FUTURE WORK}

Suggestions are made here for improvements on the current design and future work that may be done based upon what has been presented in this thesis. These suggestions will be presented by the chapter in which the topic was presented.

\section{Chapter II}

The decision to use the Xilinx family of FPGA devices was based upon the resources we have in our possession here at Portland State University. Future work might include investigating other FPGA families such as those produced by Actel, AT\&T and Atmel. These families may implement the design of the CCM much more efficiently, however, the devices and development tools would have to be acquired through some type of donation. Other areas of interest would be to explore additional logic synthesis tools that may interface with the Xilinx devices we currently have. If development tools were acquired and installed on the Sun workstations, a larger number of students would be able to take advantage of what FPGAs have to offer.

\section{Chapter III}

The current class of cube calculus operations executed upon the CCM2 covers a wide range of applications. However, with the addition of a wider class of operations, the more applications needing the minimization of logic expressions may be implemented. The CCM3 architecture has been adapted to work on extensions of the operations presented in this thesis, including the ability to operate upon symbols. Adding more operations to the CCM's library will only add to its flexibility. 


\section{Chapter IV}

This chapter presents the CCM2 architecture and how it is currently realized in logic. Future machines will expand upon this design allowing a greater amount of inputs and outputs per IT. Additional work on the CCM2 might include the implementation of the counter used to check for contradictions within operations upon two cubes. The current ILU design implements a successive counter with a pseudo-random count output. This was included to allow its accommodation within the ILU programmed design, but is not implemented since an external decoder and encoder are needed to inform the control unit $(\mathrm{CU})$ of a detected contradiction.

Additional work other than extensions to the ILU include the realization of a bus interface, bus control circuitry and a control unit to handle the necessary global signals between a host computer and the CCM2. Data and instruction registers also need to be constructed. These are large projects within themselves and may be done in class projects or as future thesis work.

\section{Chapter V}

The current demonstration board that I have constructed is merely for the testing of the ILU. The current design is a 16-bit ILU shared between two LCA devices and only consumes 40 percent of each device. One of the first things that should be done is to extend the ILU to a 32-bit machine. This would double the size of the design, consuming 80 percent of each device. Currenly, 51 percent of the I/O Blocks (I/OBs) are being used for this design. By doubling the size of the ILU design, an additional $32 \mathrm{I} / \mathrm{OBs}$ are needed pushing the total IOB consumption to 75 percent. This design change may be made in a short period of time without many problems. Additional wiring would be needed as well as 6 additional dip-switch banks and 16 LEDs to handle the inputs and outputs respectively. This will take some to organize since the majority of user $\mathrm{V} / \mathrm{O}$ pins 
becomes more scarce. This addition makes the CCM scalable in design by 16 bits. Future application needs may require the CCM2 to expand to handle input cubes of 64 bits.

The design needs to eventually be moved to a board that will accommodate the bus interface circuitry and CU to interface with a host computer. Software would then need to be designed to allow a user to input cubes and receive resultant values back from the board, storing the values in specified registers. If the design is not improved through additions and modifications to the design, it will still serve a purpose as a learning tool for future classes wanting to learn about general purpose logic computer architectures. 


\section{REFERENCES}

1 Akers, S.B., "Binary Decision Diagrams," IEEE Trans. on Computers, Vol. C-27, No. 6, June, 1978, pp. 509-516.

2 Antola, A., Avai, A., Breveglieri, L., Paparella, A., "Modular Design Methodologies for Image Processing Architectures," 6th Intl. Conf. on VLSI Design, January, 1993.

3 Brace, K.S., Rudell, R.L., Bryant, R.E., "Efficient Implementation of a BDD Package," Proc. 27th ACM/IEEE DAC, June 1990, pp. 40-45.

4 Brayton, R.K., McMullen, C., Hachtel, G.D., Sangiovanni-Vincentelli, A., Logic Minimization Algorithms for VLSI Synthesis, Kluwer Academic Publishing Co., Boston, 1984.

5 Brayton, R.K., Rudell, R., Sangiovanni-Vincentelli, A., Wang, A.R., "MIS: A Multiple-Level Logic Optimization System," IEEE Trans. on Computer-Aided Design, Vol. CAD-6. No. 6, pp. 1062-1081, November 1987.

6 Bryant, R.E., "Symbolic Manipulation of Boolean Functions Using a Graphical Representation," Proc. IEEE 22nd Design Automation Conference, 1985, pp. 688-694.

7 Chakravarty, S., "A Characterization of Binary Decision Diagrams," IEEE Trans. on Computers, Vol. 42, No. 2, pp. 129-137, February 1993.

8 Chan, A.H., "Using Decision Trees to Derive the Complement of a Binary Function with Multiple-Valued Inputs," IEEE Trans. on Computers, Vol. C-36, No. 2, February, 1987, pp. 212-214.

9 Chan, P.K., Schlag, M.D.F., Martin, M., "BORG: A Reconfigurable Prototyping Board using Field-Programmable Gate Arrays," ACM Intl. Workshop on FPGA, UC Berkeley, CA, Feb. 17-18, 1992.

10 Chuang, H.Y.H., and Li, C.C., "A Systolic Array Processor for Straight Line Detection by Modified Hough Transform," Proc. IEEE Comput. Soc. Workshop Computer Architecture for Pattern Analysis and Image Database Management, 1985 .

11 Clark, T.R., "Fitting Programmable Logic," IEEE Spectrum, March 1992, pp. 36-39. 
12 Dietmeyer, D.L., Logic Design of Digital Systems (2nd Edition), Allyn and Bacon Inc., Boston MA, 1978.

13 Engelbarts, C., "The Multiple-Valued Cube Calculus Machine Version 2.5," Department of Electrical Engineering, Portland State University, August 1993.

14 Filo, D., Yang, J.C.Y., Mailhot, F., De Micheli, G., "Technology Mapping for a Two-Output RAM-Based Field Programmable Gate Array," IEEE, 1991.

15 Falkowski, B.J., Perkowski, M.A., "Algorithm for the generation of disjoint cubes for completely and incompletely specified boolean functions," Int. Journal of Electronics, Vol. 70, No. 3, 1991, 533-538.

16 Felt, E., York, G., Brayton, R., Sangiovanni-Vincentelli, A., "Dynamic Variable Reordering for BDD Minimization," Proc. IEEE, pp. 130-135.

17 Fleisher, H., Tavel, M., Yeager, J., "Exclusive-OR Representations of Boolean Functions," IBM Journal of Research and Development, Vol. , pp 412-415, March 16, 1982.

18 Francis, R., Rose, J., Vranesic, Z., "Chortle-crf: Fast Technology Mapping for Lookup Table-Based FPGAs," Proc. 28th ACM/IEEE Design Automation Conference.

19 Gray, C.T., Liu, W., Hughes, T., Cavin, R., "The Design of a High-Performance Scalable Architecture for Image Processing Applications," IEEE Int. Conf. on Application Specific Array Processors, 1990.

20 Hall, D.V., "Introduction to Binary Decision Diagrams," Report, Portland State University, Winter 1992.

21 Hartenstein, R.W., Hirshbiel, A., Weber, M., "XPUTERS: An Open Family of Non-von Neumann Architectures," Universitat Kaiserslautern, Fachbereich Informatik, Postfach 3049, D-6750 Kaiserslautern, Germany, 1989.

22 Hartenstein, R.W., Hirshbiel, A., Reinig, H., Riedmuller, M., Schmidt, K., Weber, M., "XPUTERS: A New R \& D Area," Universitat Kaiserslautern, Fachbereich Informatik, Postfach 3049, D-6750 Kaiserslautern, Germany, 1990.

23 Hartenstein, R.W., Hirshbiel, A., Reinig, H., Riedmuller, M., Schmidt, K., Weber, M., "A Novel ASIC Design Approach Based on a New Machine Paradigm," IEEE Jornal of Solid-State Circuits, July 1991, Vol. 26, Nr. 7, pp. $1-14$.

24 Helliwell, M., Perkowski, M., "A Fast Algorithm to Minimize Mult-Output Mixed-Polarity Generalized Reed-Muller Forms," Proc. 25th ACM/IEEE Design Automation Conference, 1988, pp. 427-432. 
25 Ho, P.M., Perkowski, M.A., "Systolic architecture for Solving NP-Hard Combinatorial Problems of Logic Design and Related Areas," IEEE/ISCAS, 1989, pp. 1170-72.

26 Hong, S.J., Ostapko, D.L., "On Complementation of Boolean Functions," IEEE. Trans. on Computers, pp. 1022, September, 1972.

27 Hong, S.J., Cain, R.G., Ostapko, D.L., "MINI: A Heuristic Approach for Logic Minimization," IBM Journal of Research and Development, Vol. 18, pp 443-458, September 1974.

28 Hussain, Z., Digital Image Processing - Practical Applications of Parallel Processing Techniques, Ellis Horwood, New York, 1991.

29 Jacobi, R.P., Trullemans, A.M., "A New Logic Minimization Method for Multiplexor-Based FPGA Synthesis," Proc. IEEE, 1993, pp. 312-317.

30 Kida, L.P., Perkowski, M.A., "The Cube Calculus Machine: A Ring of Asynchronous Automata to Process Multiple-Valued Boolean Functions," Portland State University, 1990

31 Kohavi, Z., Switching and Finite Automata Theory, McGraw-Hill, New York, 1978.

32 Kreiger, R., Becker, B., Sinkovic, R., "A BDD-based Algorithm for Computation of Exact Fault Detection Probabilities," Proc. IEEE, 1993, pp. 186-195.

33 M Synthesis Users Guide, Mentor Graphics Corp., 1990.

34 Malik, S., Wang, A., Brayton, R., Sangiovanni-Vincentelli, A., "Logic Verification using Binary Decision Diagrams in a Logic Synthesis Environment," Proc. IEEE ICCAD, November 1988, pp. 6-9.

35 Moller, D., Mohnke, J., Weber, M., "Detection of Symmetry of Boolean Functions Represented by ROBDDs," Proc. IEEE, 1993, pp. 680-684.

36 Ochi, H., Yasuoka, K., Yajima, S., "Breadth-First Manipulation of Very Large Binary-Decision Diagrams," Proc. IEEE, pp. 48-54.

37 Perkowski, M.A., Brandenburg, J., "Solving Basic Boolean Algebra Problems on a Hypercube Computer," Dept. of Electrical Engineering, Portland State University.

38 Perkowski, M.A., "Systolic Architecture for the Logic Design Machine," Department of Electrical Engineering, Portland State University, 1985. 
Perkowski, M.A., Helliwell, M., Wu, P., "Minimization of Multiple-Valued Input Multi-Output Mixed-Radix Exclusive Sums of Products for Incompletely Specified Boolean Functions," Proc. 25th ACM/IEEE Des. Auto. Conf., pp. 427-432, June, 1989.

40 Perkowski, M.A., Dysko, P., Falkowski, B.J., "Two Learning Methods for a Tree-Search Combinatorial Optimizer," Proc. IEEE, 1990, pp. 606-613.

41 Perkowski, M.A., "A Synergistic Approach to Logic, Design, Computers and Problem-Solving," Ch. 22, 1991.

42 Perkowski, M.A., "The Cube Calculus Machine Number Three," Department of Electrical Engineering, Portland State University, 1991.

43 Roth, C.H., Fundamentals of Logic Design, 4th Edition, West Publishing Co., St. Paul, 1992.

44 Roth, J.P., Computer Logic, Testing and Verification, Computer Science Press, Potomac MD, 1980.

45 Rudell, R.L., Sangiovanni-Vincentelli, A.L.M., "ESPRESSO-MV: Algorithms for Multiple-Valued Logic Minimization," Proc. IEEE Custom Integrated Circuits Conference, 1985, pp. 230-234.

46 Rudell, R.L., Sangiovanni-Vincentelli, A.L.M., "Multiple-Valued Minimization for PLA Optimization," IEEE Trans. on Computer-Aided Design, Vol. CAD-6, No. 5, September 1987, pp. 727-750.

47 Sanz, J.L.C., Hinkle, E.B., Dinstein, I., "Computing Geometrical Features of Digital Objects in General Purpose Image Processing Pipeline Architectures," IBM Research, San Jose, CA, 1985.

48 Sasao, T., "An Algorithm to Derive the Complement of a Binary Function with Multiple-Valued Inputs," IEEE Trans. on Computers, Vol. C-34, No. 2, February 1985, pp. 131-140.

49 Sasao, T., Brand, D., "On the Minimization of AND-EXOR Expressions," Dept. of C.S. and E.E., Kyushu Inst. of Tech., July 20. 1990.

50 Silberberg, T.M., "The Hough Transform on the Geometric Arithmetic Parallel Processor," Proc. IEEE Conf. Computer Vision and Pattern Recognition, pp. 265-270. 1985.

51 Svoboda, A., "Boolean Analyzer," Proc. Information Processing 68, Amsterdam, North-Holland, 1969.

52 Svoboda, A., "Parallel Processing in Boolean Algebra," IEEE Trans. on Computers, Vol. C-22, No. 9, September 1973, pp. 848-851. 
53 Ulug, M.E., "VLSI Knowledge Representation Using Predicate Logic and Cubical Algebra," GE Co., New York, 1987.

54 Van Den Bout, D.E., Morris, J.N., Thomae, D., Labrozzi, S., Wingo, S., Hallman, D., "AnyBoard: An FPGA-Based, Reconfigurable System," IEEE Design and Test of Computers, Sept. 1992, pp. 21-30.

55 Weiss, R., "Flash-Programmable Complex PLD Holds Pin-to-Pin Delays to 10 nsec," EDN, March 17, 1994, pp. 93.

56 Weiss, R., "FPGA Targets Dynamically Reloadable Logic," EDN, March 17, 1994, pp. 93-94.

57 Weiss, R., "Probing the Limits of Logic Synthesis," EDN, March 17, 1994, pp. 50-62.

58 XILINX: The Programmable Logic Data Book, Xilinx Inc., San Jose, CA, 1992.

59 XILINX: The Programmable Logic Data Book, Xilinx Inc., San Jose, CA, 1993.

60 XILINX: User Guide and Tutorials, Xilinx Inc., San Jose, CA, 1991. 
APPENDIX A

CONTROL UNIT PLD CODE 
NAME

PARTNO

REVISION

DATE

DESIGNER

COMPANY

ASSEMBLY

LOCATION

DEVICE

CU.PLD;

00 ;

00 ;

4/14/94;

David W. Foote;

Portland State University;

None;

None;

GAL16V8;

\begin{abstract}
/* Control Unit
$1 *$ for the design and testing of the

/* Cube Calculus Machine II
\end{abstract}

/* INPUTS

PIN1 = CLK; /* Signal driving control unit

PIN2 $=\mathbb{R R} 0 ; / * 0=$ Sequential, $1=$ Combinational

PIN3 $=\mathbb{I R} 1 ; * 0=$ Stand-alone, $1=$ Chain

PIN4 $=\mathbb{I R} 3 ; / * 0=\mathrm{OR}$ relation, $1=\mathrm{AND}$ relation

PIN5 $=\mathbb{I R} 4 ; / * 0=\mathrm{OR}$ prerelation, $1=\mathrm{AND}$ prerelation

PIN6 $=\mathbb{I R} 5 ; / *$ IR5IIR6 $=00$ - prerelation 01 - sharp 10 - consensus $11-$ crosslink

PIN7 = IR6;

PIN8 = READY; $/ *$ READY from ILU

PIN9 = NEXTIN; $/ *$ NEXT from ILU

PIN19 = RESET; $/ *$ RESET TO STATE 0

/* OUTPUTS

PIN12 = CLEAR; / CLEAR to ILU to reset PSMs

PIN13 = REQUEST; /* REQEST to ILU to change state of PSMs

PIN14 = ANDOR; $/ *$ AND_OR to ILU to determine operation

PIN15 = NEXTOUT; $/ *$ NEXT to ILU

PIN16 = Q2;

$\mathrm{PIN} 17=\mathrm{Q} 1$

$\mathrm{PIN} 18=\mathrm{Q}$;

/* DECLARATIONS AND INTERMEDIATE VARIABLE DEFINITIONS

FIELD bstate $=[\mathrm{Q} 2, \mathrm{Q} 1, \mathrm{Q} 0] ; \quad / *$ Define Control States

DEFINE S0 ' $b$ '000

DEFINE $S 1$ ' $b$ '001

DEFINE $S 2$ 'b'011

DEFINE S3 'b'010 
DEFINE S4 'b' 110

DEFINE S5 'b' 111

DEFINE S6 'b'101

DEFINE S7 'b'100

/* LOGIC EQUATIONS */

and = (!IR0 \& !IR4 \& !IR5 \& !IR6) \# (!IR0 \& IR4 \& (IR5 \# IR6));

or = (!IR0 \& IR4 \& !IR5 \& !IR6) \# (!IR0 \& !IR4 \& (IR5 \# IR6));

sequence bstate \{

PRESENT S0 if !IR0 \& !IR1 \& !RESET NEXT S1 OUT CLEAR, REQUEST; DEFAULT NEXT S0;

PRESENT S1 if RESET NEXT S0;

if and NEXT S2 OUT ANDOR, CLEAR;

if or NEXT S2 OUT CLEAR;

PRESENT S2 if RESET NEXT S0;

if IR3 NEXT S3 OUT NEXTOUT, REQUEST, ANDOR;

if !IR3 NEXT S3 OUT NEXTOUT, REQUEST;

DEFAULT NEXT S3;

PRESENT S3 if RESET NEXT S0;

DEFAULT NEXT S4 OUT NEXTOUT;

PRESENT S4 if RESET NEXT S0;

DEFAULT NEXT S5 OUT REQUEST;

PRESENT S5 if RESET NEXT S0;

if READY NEXT S6;

DEFAULT NEXT S5;

PRESENT S6 if RESET NEXT S0;

DEFAULT NEXT S7 OUT REQUEST;

PRESENT S7 if RESET NEXT S0;

if !NEXTIN \& READY NEXT S4;

DEFAULT NEXT S0; \} 


\section{APPENDIX B}

\section{LOGIC CELL ARRAY PLACEMENT REPORT}


AUTOMATIC PLACE AND ROUTE PROGRAM -- Version 3.30

Copyright (C) 1986-1992 by Xilinx, Inc. All Rights Reserved.

Includes DOS/4G Version 1.7

Copyright (c) Rational Systems, Inc. 1990-1992

Tue May 17 16:27:03 1994

Input Design File: ilu.lca

Part Type: 3090PP175

Speed Grade: -50

Guide Design File: (none)

Schematic File: ilu.scp

Constraints File: (none)

Options: -a3 -r4 -s28759-w

Output Design File: iluapr.lca

Report File: iluapr.rpt

Message File: (none)

Placement time: $3 \mathrm{hrs}, 56$ mins, 52 secs

Routing time: $0 \mathrm{hrs}, 48 \mathrm{mins}, 21$ secs

Total elapsed time: $4 \mathrm{hrs}, 46$ mins, 11 secs

FINAL RESULTS:

Total:

Unrouted:

Blks (CLBs IOBs TBUFs PLUPs CLKs OSCs) Pins Nets Loads Nets $\begin{array}{lllllllllll}205 & 131 & 74 & 0 & 0 & 0 & 0 & 814 & 222 & 0 & 0\end{array}$

Net Routing Order:

REQUEST, CTOi, \$1-233302, \$2-CNTOi, \$2-324333, \$2-330339, \$32-S0, \$36So, \$37-D23, \$45-CNTOi, \$45-324318, \$45-327336, \$75-S0, \$79-S0, \$80-D23, \$88-CNTOi, \$88-324333, \$88-330339, \$118-S0, \$122-S0, \$123-D23, \$131CNTOi, \$131-324318, \$131-327336, \$165-S1, \$170-D23, CT3i, CT2i, CT1i, SC7, \$1-312302, \$2-324303, \$2-324318, \$2-327336, \$32-S1, \$36-S1, \$41-D23, \$45324333, \$45-330339, \$45-453300, \$84-D23, \$88-324318, \$88-327336, \$88453300, \$118-S1, \$122-S1, \$127-D23, \$131-324333, \$131-330339, \$131453300, \$161-S1, \$165-SO, \$166-D23, SC6, \$45-BEFOREi, \$88-BEFOREi, \$131-BEFOREi, CARRYip1, \$45-324303, CONFi, \$131-TESTATEi, \$131300289, CT4i, \$1-181281, \$2-453300, \$99-D01, \$161-S0, CT3i-1, \$2-POS2, \$45-POS2, \$45-TESTATEi, \$45-300289, \$88-ACTIVEi, \$88-TESTATEi, \$131POSO, \$131-POS2, \$1-260268, \$45-COUNTi, \$1-339281, \$2-TESTATEi, \$2STATE1, \$1-154302, \$1-339290, \$1-391302, \$88-324303, \$95-D01, \$131324303, \$1-181284, \$2-BEFOREi, \$45-POS3, \$88-300289, \$1-339268, \$2STATEO, CTOi-1, SC4, \$2-COUNTi, \$9-D01, \$75-S1, \$79-S1, \$138-D01, \$142D01, CT4i-1, \$1-339284, \$2-ACTIVEi, \$2-300289, \$45-ACTIVEi, \$45-POSO, 
\$131-STATE1, \$45-STATE0, \$45-STATE1, \$88-STATE0, \$131-STATE0, READYo, SC5, \$1-339293, \$131-ACTIVEi, CT1i-1, CT2i-1, AFT1, \$13-D01, \$52D01, \$56-D01, \$2-POSO, \$2-POS1, \$2-POS3, \$131-POS3, \$1-181268, \$1181287, \$88-STATE1, \$1-260274, \$1-181290, \$1-181293, \$88-POS2, \$131POS1, \$1-339287, \$1-181274, SC2, ACT1, \$131-REL1i, \$1-339274, AFT3, \$88SLCTOi, SC3, \$45-POS1, \$88-POS0, \$88-POS3, ACT3, AFT0, \$88-SLCT1i, \$131-SLCT1i, \$131-RELOi, SB2, BEF3, BEF1, T1, \$88-POS1, \$45-REL1i, \$131SLCTOi, SC0, SC1, SBO, CLEAR, AFT2, \$1-260287, \$45-RELOi, \$88-COUNTi, \$88-REL1i, ACT2, COUNT_MODE, \$2-SLCT1i, PRIME, W3, \$1-181262, \$1260262, NEXTip1, REL1, \$1-260290, \$1-260293, ACTO, REL2, RIGHT_E4, SAO, SA1, SA3, \$2-SLCTOi, \$1-260281, SB3, REL3, SA2, W1, \$1-339262, RELO, \$1-260284, \$88-RELOi, BEF2, \$45-SLCTOi, BEF0, W4, RIGHT_E3, T0, \$131-COUNTi, SB1, SB6, SA4, W2, READYi, SB4, \$2-REL1i, RIGHT_Ei-1, SA6, SA7, \$2-RELOi, SA5, \$45-SLCT1i, RIGHT_E1, RIGHT_E2, NEXTi, SB5, AND_OR, SB7, CARRYi, CONFi-1

Final Placement:

\begin{tabular}{|c|c|c|}
\hline Block Name & Location & Moved or Swapped Pins \\
\hline $\begin{array}{l}\text { ACT0 } \\
\text { ACT1 } \\
\text { ACT2 } \\
\text { ACT3 } \\
\text { AFT0 } \\
\text { AFT1 } \\
\text { AFT2 } \\
\text { AFT3 } \\
\text { AND_OR } \\
\text { BEF0 } \\
\text { BEF1 } \\
\text { BEF2 } \\
\text { BEF3 } \\
\text { CARRYi } \\
\text { CARRYip1 } \\
\text { CLB1 } \\
\text { CLB2 } \\
\text { CLB3 } \\
\text { CLB4 } \\
\text { CLB5 } \\
\text { CLB6 } \\
\text { CLB7 } \\
\text { CLB8 }\end{array}$ & $\begin{array}{l}\text { N5 } \\
\text { N6 } \\
\text { P6 } \\
\text { R6 } \\
\text { N7 } \\
\text { P7 } \\
\text { T7 } \\
\text { T6 } \\
\text { P16 } \\
\text { P4 } \\
\text { R4 } \\
\text { T4 } \\
\text { T5 } \\
\text { J1 } \\
\text { H16 } \\
\text { SG } \\
\text { NE } \\
\text { NI } \\
\text { MK } \\
\text { NJ } \\
\text { OL } \\
\text { OK } \\
\text { OM }\end{array}$ & $\begin{array}{l}A \rightarrow B, B \rightarrow C, C \rightarrow E, E \rightarrow A \\
A \rightarrow C, B \rightarrow A, C \rightarrow E, E \rightarrow B \\
A \rightarrow D, B \rightarrow E, D \rightarrow B, E \rightarrow A \\
X \ll Y, B \rightarrow E, D \rightarrow B, E \rightarrow D \\
B \rightarrow E, C \rightarrow B, E \rightarrow C \\
X \ll Y, A<->D, B<->E \\
A \ll D, B<->E \\
A \rightarrow D, B \rightarrow A, D \rightarrow E, E \rightarrow B\end{array}$ \\
\hline
\end{tabular}




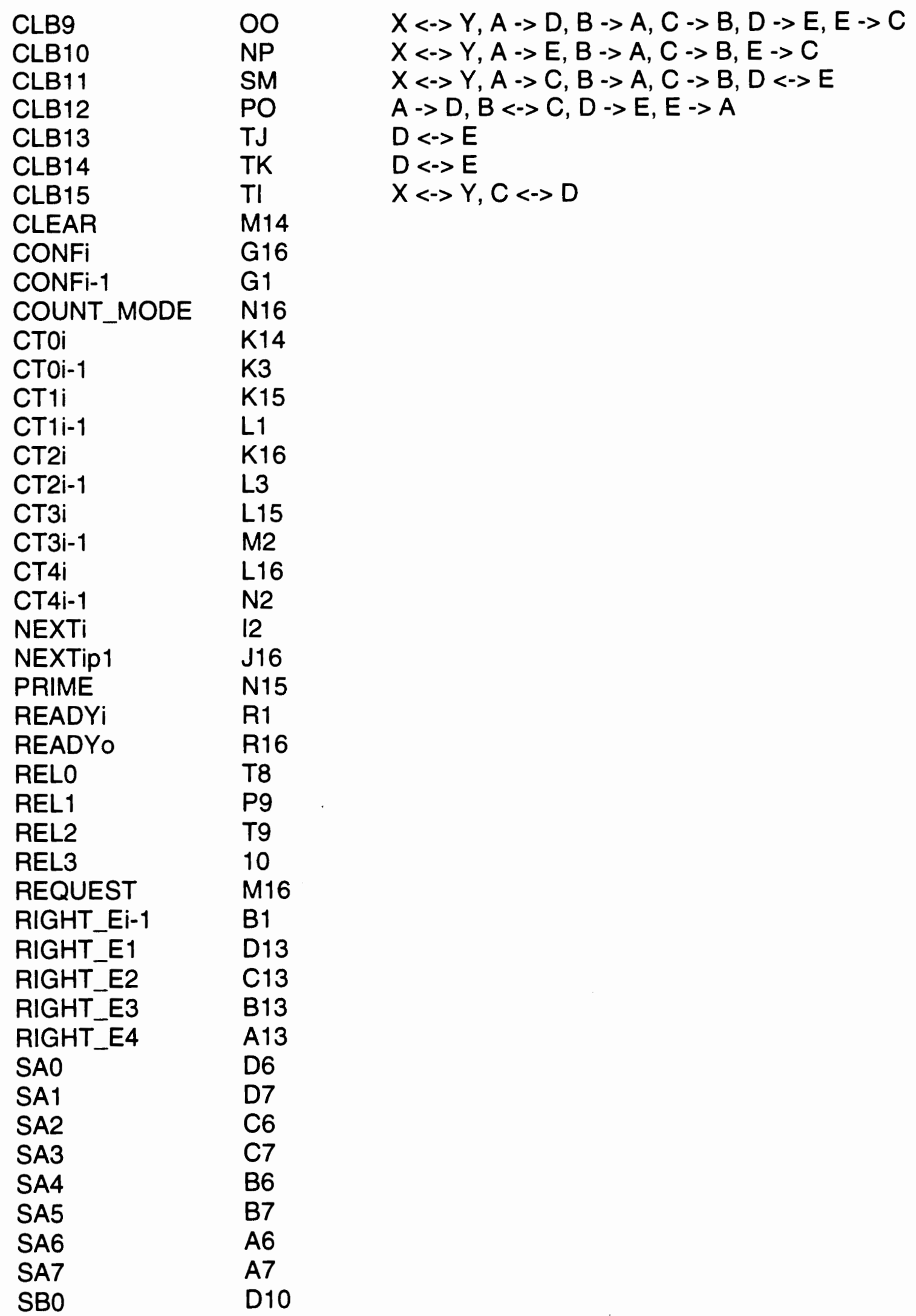




\begin{tabular}{|c|c|c|}
\hline $\begin{array}{l}\text { SB1 } \\
\text { SB2 } \\
\text { SB3 } \\
\text { SB4 } \\
\text { SB5 } \\
\text { SB6 } \\
\text { SB7 } \\
\text { SC0 } \\
\text { SC1 } \\
\text { SC2 } \\
\text { SC3 } \\
\text { SC4 } \\
\text { SC5 } \\
\text { SC6 } \\
\text { SC7 } \\
\text { T0 } \\
\text { T1 } \\
\text { W1 } \\
\text { W2 } \\
\text { W3 } \\
\text { W4 }\end{array}$ & $\begin{array}{l}\text { D11 } \\
\text { C10 } \\
\text { C11 } \\
\text { B10 } \\
\text { B11 } \\
\text { A10 } \\
\text { A11 } \\
\text { P13 } \\
\text { R13 } \\
\text { T13 } \\
\text { R12 } \\
\text { T12 } \\
\text { N11 } \\
\text { P11 } \\
\text { R11 } \\
\text { N10 } \\
\text { P10 } \\
\text { D12 } \\
\text { C12 } \\
\text { B12 } \\
\text { A12 }\end{array}$ & ' \\
\hline
\end{tabular}


APPENDIX C

LOGIC CELL ARRAY TIMING DELAYS 
XDelay: ILUAPR.LCA (3090PP175-50), XACT 4.30, Wed Aug 3 04:46:08 1994

Xdelay path report options:

From IOB "NEXTi" (J2)

To IOB "NEXTip1" (J16)

TO "\$131-POS3.E"

To "\$131-POS3.C"

To "\$131-POS3.B"

To "\$131-POS3.D"

To "\$131-POS3.A"

Output will be sorted by decreasing path delays.

Logical Path

From: BIk NEXTi

Thru: Net NEXTi

Thru: Blk \$1-181274

Thru: Net \$1-181274

Thru: Blk \$1-260274

Thru: Net \$1-260274

Thru: Blk \$1-339274

Thru: Net \$1-339274

Thru: BlK CLB10

Thru: Net NEXTip1
Delay Cumulative

\begin{tabular}{|c|c|}
\hline $\begin{array}{l}\text { AD to J2.I } \\
\text { to DH.B } \\
\text { to DH.X } \\
\text { to MH.E } \\
\text { to MH.Y } \\
\text { to PH.E } \\
\text { to PH.X } \\
\text { to RJ.C } \\
\text { to RJ.X } \\
\text { to J16.O } \\
\text { ETip1 }\end{array}$ & 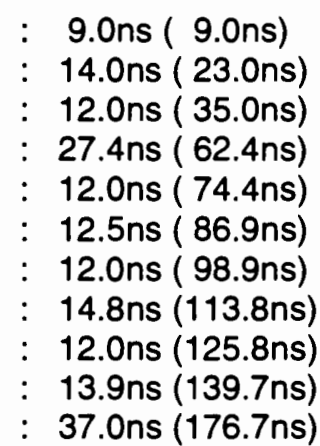 \\
\hline
\end{tabular}

Xdelay path report options:

From IOB "READYi" (R1)

To IOB "READYo" (R16)

TO "\$131-POS3.E"

To "\$131-POS3.C"

To "\$131-POS3.B"

To "\$131-POS3.D"

To "\$131-POS3.A"

Output will be sorted by decreasing path delays.

Logical Path

From: Blk READYi

Thru: Net READYi

Thru: BlK CLB11

Thru: Net READYo

To: O pin to PAD,

\begin{tabular}{|c|c|}
\hline \multicolumn{2}{|c|}{ Delay Cumulative } \\
\hline $\begin{array}{l}\text { PAD to R1.I } \\
\text { to } M L . A \\
\text { to } M L . Y \\
\text { to } R 16.0\end{array}$ & $\begin{array}{c}9.0 \mathrm{~ns}(9.0 \mathrm{~ns}) \\
: 36.3 \mathrm{~ns}(45.3 \mathrm{~ns}) \\
: 12.0 \mathrm{~ns}(57.3 \mathrm{~ns}) \\
: \quad 15.3 \mathrm{~ns}(72.7 \mathrm{~ns}) \\
: \quad 37.0 \mathrm{~ns} \text { (109.7ns) }\end{array}$ \\
\hline
\end{tabular}


Xdelay path report options:

Input delays

Logical Path

Delay Cumulative

From: BIk BEFO

Thru: Net BEFO

$$
\begin{aligned}
& \text { PAD to P4.I : 9.0ns (9.0ns) } \\
& \text { to KO.B : 16.1ns (25.1ns) }
\end{aligned}
$$

To: Blk \$45-POSO

From: Blk \$45-POSO

To: BIK CLB6

From: Blk CLB6

Thru: Net SC5

to OM.X : $\quad$ O.Ons ( $0.0 \mathrm{~ns})$

to N11.O : 7.0ns (7.0ns)

To: O pin to PAD, Blk SC5

: 37.0ns (44.0ns)

(101.3ns)

From: Blk \$45-POSO

To: Blk CLB7

From: Blk CLB7

to OL.X : 0.0 ns ( 0.0ns)

Thru: Net SC4

to T12.0 : 15.8ns (15.8ns)

To: O pin to PAD, Blk SC4

: 37.0ns ( $52.8 \mathrm{~ns})$

(105.3ns)

From: Blk BEFO

Thru: Net BEFO

$$
\begin{array}{cl}
\text { PAD to P4.I } & : \text { 9.0ns ( } 9.0 n s) \\
\text { to JO.B } & : \text { 15.6ns (24.6ns) } \\
& : \text { O.Ons (24.6ns) }
\end{array}
$$

To: Blk \$2-POSO

From: Blk \$2-POSO

To: Blk CLB8

From: Blk CLB8

to NL.X : $0.0 n s$ ( $0.0 \mathrm{~ns})$

Thru: Net SC7

to R11.0 : 10.7ns (10.7ns)

To: O pin to PAD, Blk SC7

: 37.0ns (47.7ns)

(103.6ns)

From: Blk \$2-POSO

To: Blk CLB9

From: Blk CLB9

Thru: Net SC6

To: O pin to PAD, Blk SC6

to ONX : $\quad$ (27.9ns)

to P11.0 : 4.7ns (4.7ns)

: $37.0 \mathrm{~ns}$ ( $41.7 \mathrm{~ns})$

( $94.2 n s)$

From: Blk BEFO

Thru: Net BEFO

$$
\begin{array}{cl}
\text { PAD to P4.I } & : \text { 9.0ns (9.0ns) } \\
\text { to IO.B } & : \text { 15.6ns (24.6ns) } \\
& : \text { o.0ns (24.6ns) }
\end{array}
$$

Blk \$88-POSO

To: Blk CLB4

From: Blk CLB4

Thru: Net SC3

to MM.X : 0.0 ns ( $0.0 n s)$

To: O pin to PAD, Blk SC3

to R12.0 : 15.0ns (15.0ns)

From: Blk \$88-POSO

(108.3ns) 
To: Blk CLB5

From: BIk CLB5

Thru: Net SC2

To: O pin to PAD, Blk SC2

to ML.X : 0. Ons ( 0.0 ns)

to T13.0 : $22.5 \mathrm{~ns}$ (22.5ns)

: 37.0ns ( $59.5 \mathrm{~ns})$

(115.1ns)

From: BIk BEFO

Thru: Net BEFO

PAD to P4.I : 9.0ns (9.0ns)

to GO.B : $15.6 \mathrm{~ns}$ (24.6ns)

To: Blk \$131-POSO

: $0.0 n s(24.6 n s)$

From: Blk \$131-POSO

To: BIK CLB2

From: BIK CLB2

to HL.X : $0.0 n s$ ( 0.0ns)

Thru: Net SC1

To: O pin to PAD, Blk SC1

to R13.0 : $56.5 \mathrm{~ns}$ ( $56.5 \mathrm{~ns})$

: 37.0ns (93.5ns)

(145.8ns)

From: Blk \$131-POSO

To: BIK CLB3

From: Blk CLB3

Thru: Net SCO

to HO.Y : O.Ons ( 0.0 ns)

to P13.0 : 20.8ns (20.8ns)

To: O pin to PAD, Blk SCO

37.0ns ( $57.8 \mathrm{~ns})$

(101.3ns)

From: Blk BEF1

Thru: Net BEF1

PAD to R4.1 : 9.0ns ( 9.0ns)

To: Blk \$45-POS1

to KP.B : $14.4 \mathrm{~ns}(23.4 \mathrm{~ns})$

: $0.0 n s(23.4 n s)$

From: Blk \$45-POS1

To: Blk CLB6

From: Blk CLB6

Thru: Net SC5

To: O pin to PAD, Blk SC5

to OM.X : $\quad$ O.Ons ( $0.0 \mathrm{~ns}$ )

to N11.O : 7.0ns (7.0ns)

: 37.0ns (44.0ns)

(101.8ns)

From: Blk \$45-POS1

To: Blk CLB7

From: Blk CLB7

Thru: Net SC4

To: O pin to PAD, Blk SC4

to OL.X : $\quad$ O.Ons ( $0.0 n s)$

to T12.0 : 15.8ns (15.8ns)

: 37.0ns (52.8ns)

(99.3ns)

From: Blk BEF1

Thru: Net BEF1

$$
\begin{array}{ccc}
\text { PAD to R4.1 } & : & \text { 9.0ns ( } 9.0 n s) \\
\text { to JP.B } & : & 13.9 n s(22.9 n s) \\
& : & 0.0 n s(22.9 n s)
\end{array}
$$

To: Blk \$2-POS1

From: Blk \$2-POS1

To: Blk CLB8

From: Blk CLB8

Thru: Net SC7

To: O pin to PAD, Blk SC7

to NL.X : $0.0 \mathrm{~ns}$ ( $0.0 \mathrm{~ns}$ )

to R11.0 : 10.7ns (10.7ns)

: 37.0ns (47.7ns)

(104.8ns)

From: Blk \$2-POS1

To: Blk CLB9

From: Blk CLB9

Thru: Net SC6

to ON.X : 0.0 ns ( 0.0 ns)

to P11.0 : 4.7ns (4.7ns) 
To: O pin to PAD, Blk SC6

From: Blk BEF1

Thru: Net BEF1

TO: BIK \$88-POS1
: $37.0 \mathrm{~ns}(41.7 \mathrm{~ns})$

(98.2ns)

PAD to R4.I : $9.0 \mathrm{~ns}$ ( $9.0 \mathrm{~ns}$ )

to IP.B : $13.9 \mathrm{~ns}$ (22.9ns)

: $0.0 n s(22.9 n s)$

From: Blk \$88-POS1

To: Blk CLB4

From: Blk CLB4

Thru: Net SC3

To: O pin to PAD, BIK SC3

to MM.X : 0.0 ns ( $0.0 \mathrm{~ns})$

to R12.0 : 15.0ns (15.0ns)

: $37.0 \mathrm{~ns}$ ( $52.0 \mathrm{~ns})$

(103.7ns)

From: BIk \$88-POS1

To: BlK CLB5

From: Blk CLB5

Thru: Net SC2

To: O pin to PAD, Blk SC2

to ML.X : $0.0 n s$ ( $0.0 n s)$

to T13.0 : 22.5ns (22.5ns)

: 37.0ns ( $59.5 \mathrm{~ns})$

(112.5ns)

From: Blk BEF1

Thru: Net BEF1

PAD to R4.I : 9.0ns (9.0ns)

To: Blk \$131-POS1

to HP.B : $13.9 \mathrm{~ns}(22.9 \mathrm{~ns})$

: $0.0 n s(22.9 n s)$

From: Blk \$131-POS1

To: BIK CLB2

From: Blk CLB2 $\quad$ to HL.X : $0.0 \mathrm{~ns}$ ( $0.0 \mathrm{~ns}$ )

Thru: Net SC1 to R13.O : $56.5 \mathrm{~ns}$ ( $56.5 \mathrm{~ns})$

To: O pin to PAD, Blk SC1 : 37.0ns (93.5ns)

(142.6ns)

From: Blk \$131-POS1

To: BIK CLB3

From: Blk CLB3 to HO.Y : $0.0 n s$ ( 0.0ns)

Thru: Net SCO to P13.0 : 20.8ns (20.8ns)

To: O pin to PAD, Blk SCO : : 37.0ns (57.8ns)

(100.2ns)

From: Blk BEF2

Thru: Net BEF2

PAD to T4.1 : 9.0ns (9.0ns)

To: Blk \$45-POS2

to KM.B : 20.9ns (29.9ns)

: $0.0 \mathrm{~ns}(29.9 \mathrm{~ns})$

From: Blk \$45-POS2

To: Blk CLB6

From: Blk CLB6 to OM.X : $0.0 \mathrm{~ns}$ ( $0.0 \mathrm{~ns}$ )

Thru: Net SC5 to N11.0 : 7.0ns (7.0ns)

To: O pin to PAD, Blk SC5 : 37.0ns (44.0ns)

$(110.5 \mathrm{~ns})$

From: Blk \$45-POS2

To: Blk CLB7

From: Blk CLB7 to OL.X : 0.0 ns ( 0.0 ns)

Thru: Net SC4 to T12.0 : 15.8ns (15.8ns)

To: O pin to PAD, Blk SC4 : $37.0 \mathrm{~ns}$ ( $52.8 \mathrm{~ns}$ )

(107.7ns) 
From: Blk BEF2

Thru: Net BEF2

To: BIk \$2-POS2

From: Blk \$2-POS2

To: BIK CLB8

From: Blk CLB8

Thru: Net SC7

To: O pin to PAD, Blk SC7

From: Blk \$2-POS2

To: BIK CLB9

From: BIK CLB9

Thru: Net SC6

To: O pin to PAD, Blk SC6

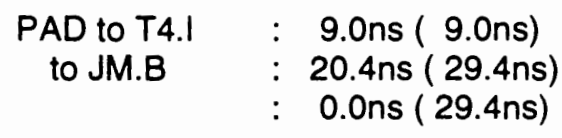

From: BIK BEF2

Thru: Net BEF2

PAD to T4.1 : 9.0 ns (9.0ns)

to ON.X : 0. Ons ( O.Ons)

to P11.0 : 4.7ns (4.7ns)

to NL.X : $\quad$ O.Ons ( $0.0 n s)$

to R11.0 : 10.7ns (10.7ns)

: 37.0ns (47.7ns)

(102.9ns)

To: Blk \$88-POS2

to IM.B : $20.4 \mathrm{~ns}(29.4 \mathrm{~ns})$

: $0.0 n s(29.4 \mathrm{~ns})$

From: Blk \$88-POS2

To: BIk CLB4

From: Blk CLB4

Thru: Net SC3

To: O pin to PAD, Blk SC3

to MM.X : $0.0 \mathrm{~ns}(0.0 \mathrm{~ns})$

to R12.0 : 15.0ns (15.0ns)

(89.2ns)

From: Blk \$88-POS2

To: BIK CLB5

From: Blk CLB5

Thru: Net SC2

To: O pin to PAD, Blk SC2

to ML.X : $\quad 0.0 n s$ ( $0.0 n s)$

to T13.0 : $22.5 \mathrm{~ns}(22.5 \mathrm{~ns})$

(119.9ns)

(121.0ns)

From: Blk BEF2

Thru: Net BEF2

PAD to T4.1 : 9.0ns (9.0ns)

to FM.B : $20.4 \mathrm{~ns}(29.4 \mathrm{~ns})$

To: Blk \$131-POS2

: $0.0 \mathrm{~ns}(29.4 \mathrm{~ns})$

From: Blk \$131-POS2

To: BIK CLB2

From: Blk CLB2

Thru: Net SC1

To: O pin to PAD, Blk SC1

to HL.X : $0.0 \mathrm{~ns}$ ( 0.0ns)

to R13.0 : $56.5 \mathrm{~ns}$ ( $56.5 \mathrm{~ns}$ )

: 37.0ns ( $93.5 \mathrm{~ns})$

(142.3ns)

From: Blk \$131-POS2

To: BIK CLB3

From: Blk CLB3

Thru: Net SCO

to HO.Y : O.Ons ( $0.0 n s$ )

to P13.0 : 20.8ns (20.8ns)

To: O pin to PAD, Blk SCO

: $37.0 \mathrm{~ns}$ ( $57.8 \mathrm{~ns})$

(106.4ns)

From: BIK BEF3

Thru: Net BEF3

PAD to T5.1 : 9.0ns (9.0ns)

to KN.B : $18.5 \mathrm{~ns}(27.5 \mathrm{~ns})$

To: Blk \$45-POS3

: $0.0 n s$ (27.5ns) 
From: Blk \$45-POS3

To: Blk CLB6

From: Blk CLB6

Thru: Net SC5

To: O pin to PAD, Blk SC5

$\begin{array}{ll}\text { to OM.X } & : \text { O.Ons ( O.Ons) } \\ \text { to N11.O } & : \text { 7.0ns ( 7.0ns) } \\ \text { C5 } & : \text { 37.0ns (44.0ns) }\end{array}$

(108.8ns)

From: Blk \$45-POS3

To: Blk CLB7

From: Blk CLB7

Thru: Net SC4

To: O pin to PAD, Blk SC4

to OL.X : 0. Ons ( 0.0 ns)

to $\mathrm{T} 12.0$ : $15.8 \mathrm{~ns}(15.8 \mathrm{~ns})$

: 37.0ns (52.8ns)

(102.7ns)

From: Blk BEF3

Thru: Net BEF3

$\begin{array}{ccc}\text { PAD to T5.I } & : \text { 9.0ns (9.0ns) } \\ \text { to JN.B } & : \text { 18.0ns (27.0ns) } \\ & : \text { o.Ons (27.0ns) }\end{array}$

To: Blk \$2-POS3

From: Blk \$2-POS3

To: BIK CLB8

From: Blk CLB8

Thru: Net SC7

To: O pin to PAD, Blk SC7

to NL.X : 0.0 ns ( $0.0 n s)$

to R11.0 : 10.7ns (10.7ns)

From: Blk \$2-POS3

To: BIK CLB9

From: Blk CLB9

Thru: Net SC6

To: O pin to PAD, Blk SC6

$$
\begin{aligned}
& \text { to ON.X : } 0.0 \text { ns ( } 0.0 n s) \\
& \text { to P11.0 : 4.7ns ( } 4.7 \mathrm{~ns}) \\
& \text { : 37.0ns (41.7ns) }
\end{aligned}
$$

From: Blk BEF3

Thru: Net BEF3

To: Blk \$88-POS3

$$
\begin{aligned}
& \text { PAD to T5.I : 9.0ns (9.0ns) } \\
& \text { to IN.B : 18.0ns (27.0ns) } \\
& \text { : } 0.0 n s(27.0 \mathrm{~ns})
\end{aligned}
$$

From: Blk \$88-POS3

To: Blk CLB4

From: Blk CLB4

Thru: Net SC3

To: O pin to PAD, Blk SC3

to MM.X : $\quad 0.0 \mathrm{~ns}(0.0 \mathrm{~ns})$

to R12.0 : 15.0ns (15.0ns)

: 37.0ns (52.0ns)

(123.3ns)

From: Blk \$88-POS3

To: Blk CLB5

From: Blk CLB5

Thru: Net SC2

To: O pin to PAD, Blk SC2

to ML.X : 0.0 ns ( $0.0 \mathrm{~ns}$ )

to T13.0 : $22.5 \mathrm{~ns}(22.5 \mathrm{~ns})$

: $37.0 \mathrm{~ns}$ ( $59.5 \mathrm{~ns})$

(120.8ns)

From: Blk BEF3

Thru: Net BEF3

To: Blk \$131-POS3

$$
\begin{array}{cc}
\text { PAD to T5. } & : \text { 9.0ns ( } 9.0 \mathrm{~ns}) \\
\text { to GN.B } & : \text { 18.0ns (27.0ns) } \\
& : \text { o.0ns (27.0ns) }
\end{array}
$$

From: Blk \$131-POS3

To: Blk CLB2 


$$
\begin{aligned}
& \text { From: BIK CLB2 to HL.X : } 0.0 \mathrm{~ns} \text { ( } 0.0 \mathrm{~ns} \text { ) } \\
& \text { Thru: Net SC1 to R13.O : 56.5ns ( } 56.5 \mathrm{~ns}) \\
& \text { To: O pin to PAD, Blk SC1 : 37.0ns ( } 93.5 \mathrm{~ns})
\end{aligned}
$$

From: Blk \$131-POS3

To: Blk CLB3

From: BIk CLB3

to HO.Y : 0.0 ns ( $0.0 n s)$

Thru: Net SCO

to P13.0 : 20.8ns (20.8ns)

To: O pin to PAD, Blk SCO

: 37.0ns ( $57.8 \mathrm{~ns})$

(103.6ns) 


\section{APPENDIX D}

SHARP SIMULATION CODE 


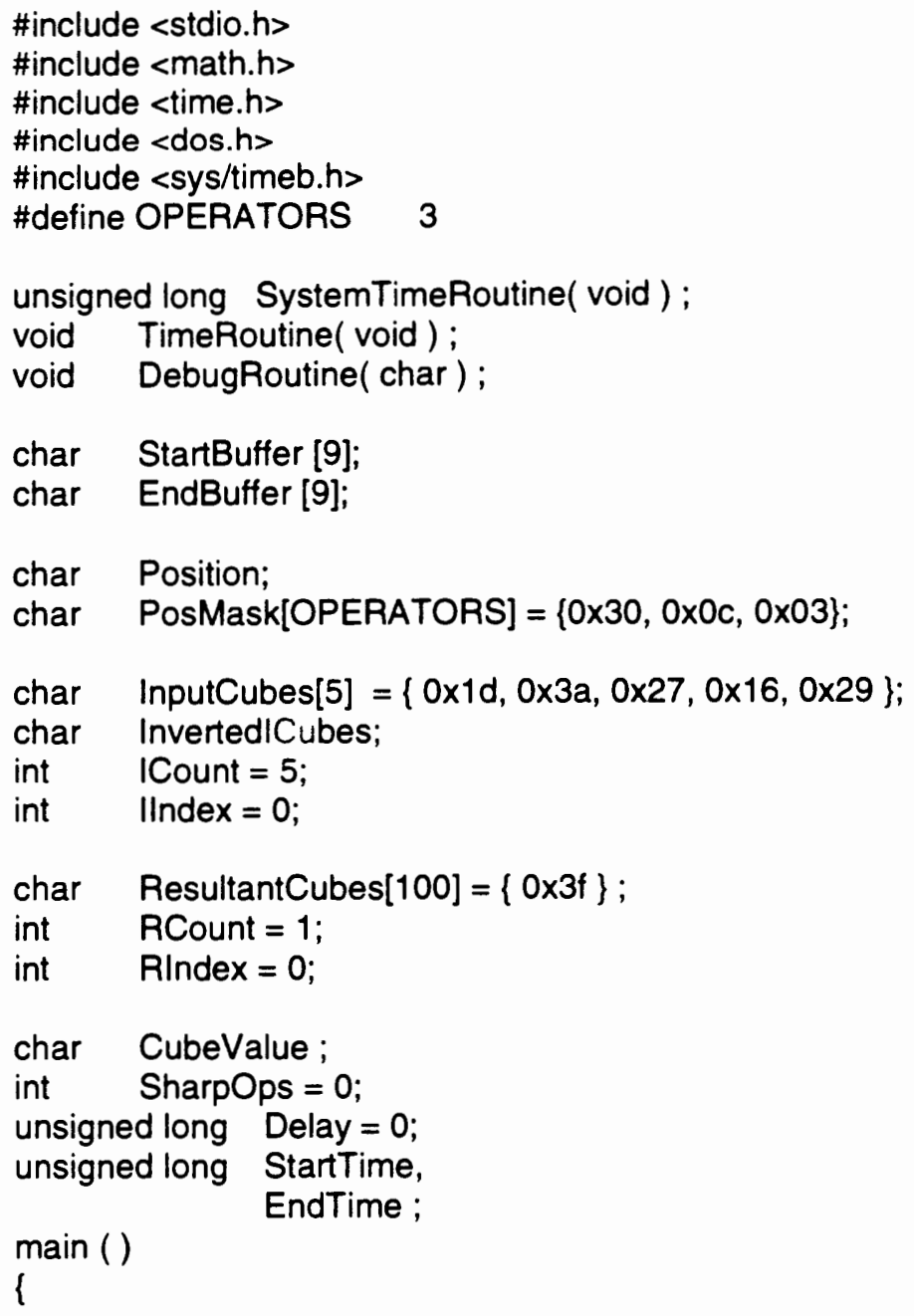




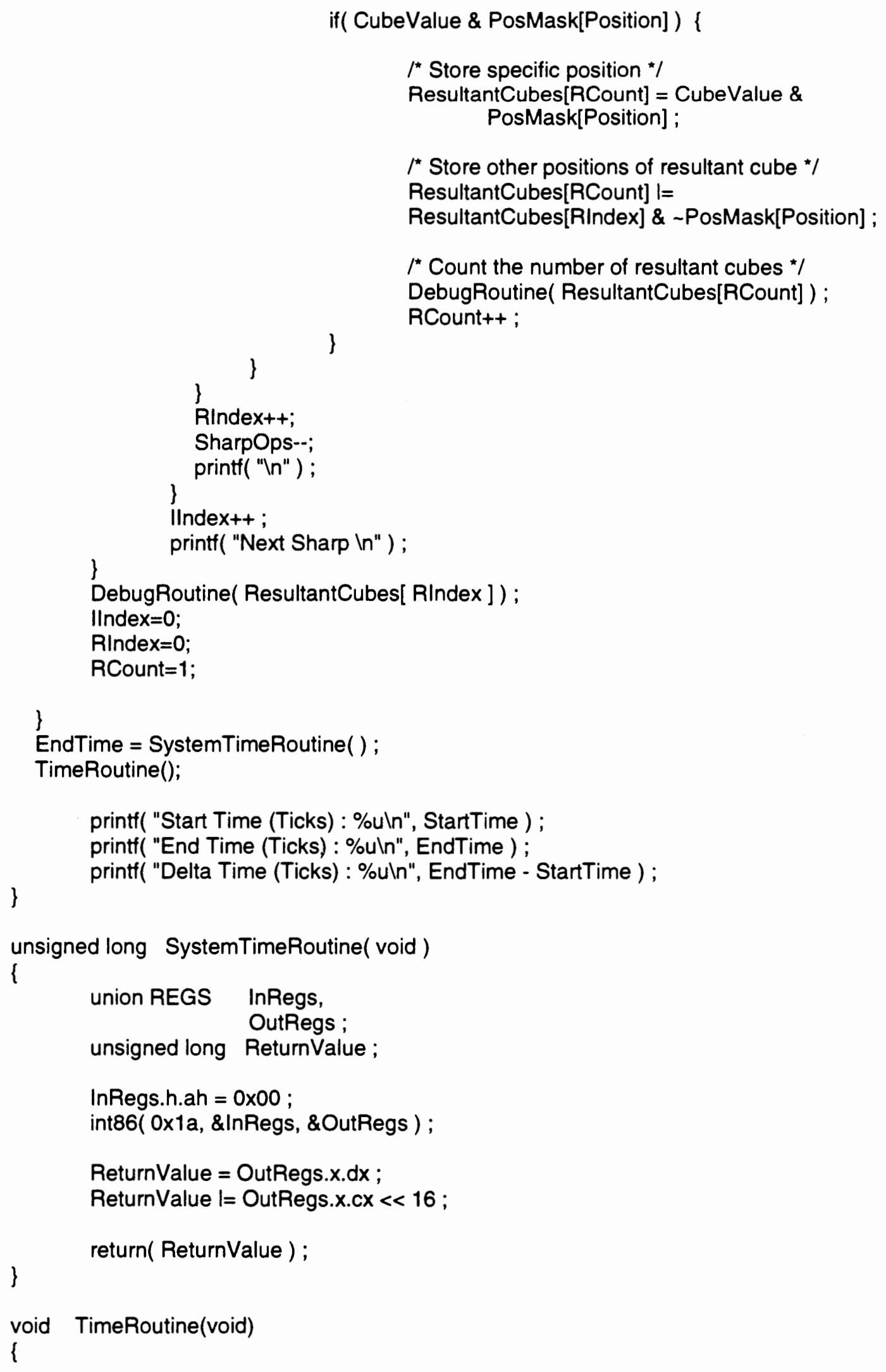




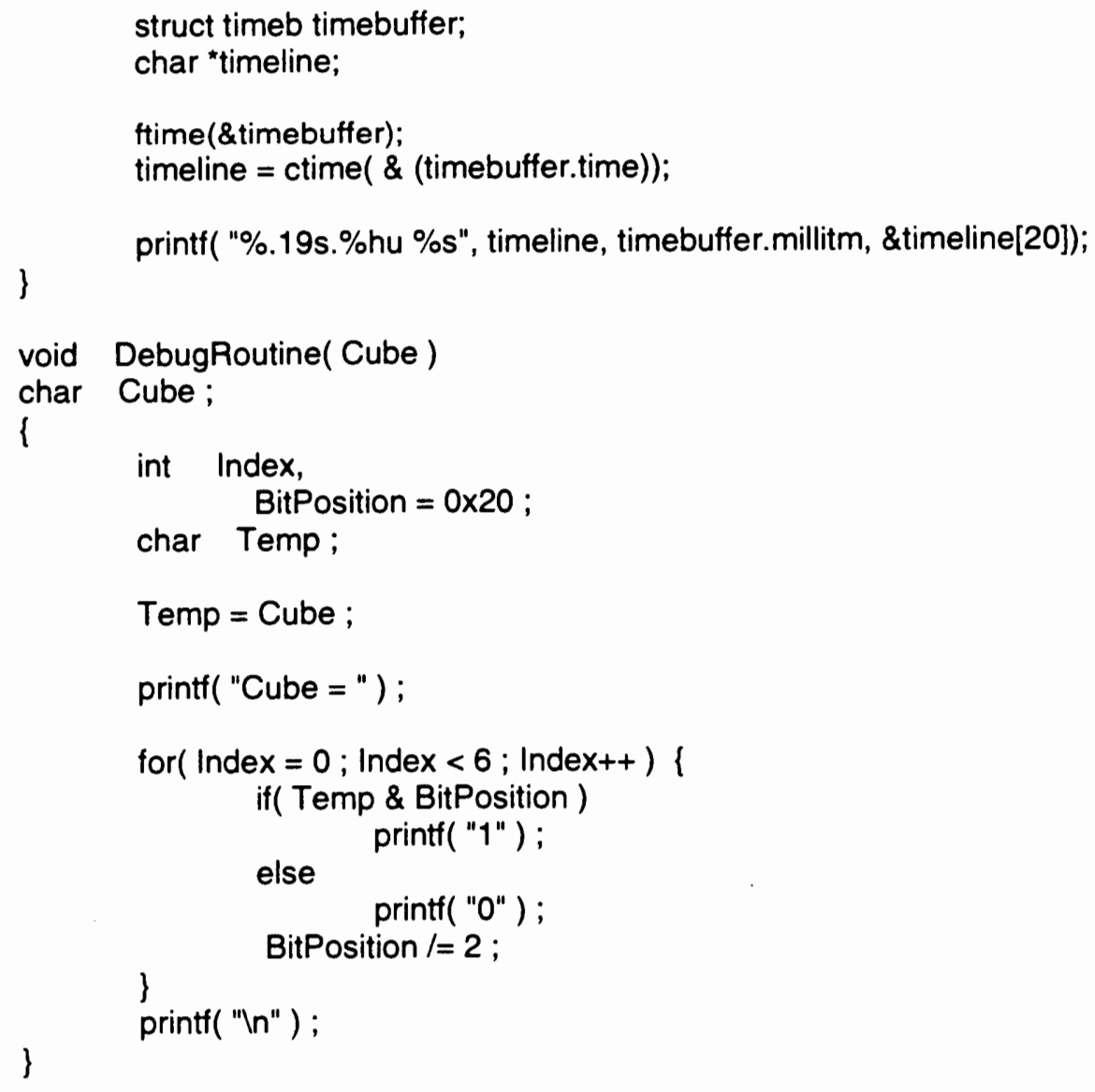


APPENDIX E

XACT DEVELOPMENT SYSTEM TUTORIAL 
The tutorial presented here is an introduction to the design flow and development tools for configuring Xilinx LCAs. The tutorial consists of a simple design for a sevensegment decoder that will be downloaded to the provided demonstration board supplied by Xilinx. It encompasses the viewing of the created design in the FutureNet DASH schematic capture package; the conversion of the drawing files to Xilinx netlist files; the mapping of the netlist files to logic cell array files; the viewing of the LCA design and layout; and the downloading and operation of the design in the Xilinx LCA. The Xilinx tools reside on the 486 PC in Dr. Perkowski's lab downstairs in the front room of the lab. The FutureNet DASH and Xilinx manuals also reside in the front room and Xilinx download cable and Demo board are stored in the back room.

\section{CREATING THE DESIGN}

The design used in this tutorial was created using the supplied schematic capture application FutureNet DASH [60]. The dashdice design is a two-level schematic, with the top-level design calling a lower-level schematic design which holds the operation of the included macro. To view the Dashdice example, you must first enter the FutureNet DASH Schematic Capture tools. To invoke DASH, type: C: $>\backslash$ fn 2 This automatically calls the macro library for the XC2000 family of LCA's. This family is selected since the demonstration board was designed for use with a Xilinx 2064 LCA device. The schematic capture package will be invoked, load the XC2000 macros, load the current profile, then give you control of the program.

The mouse is setup as such:

$$
\text { left button }=\text { select, } \text { middle button }=\text { execute, } \text { right button }=\text { menu }
$$

From the command prompt, type "dir" to view the current directory in which you are 
located, which should be C: $>$ XXACTDESIGNS. You will need to change directories to

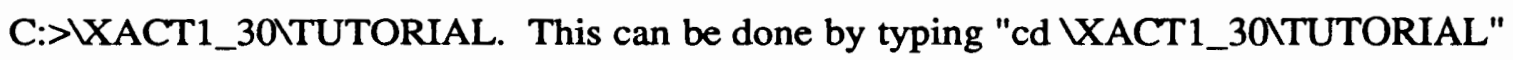
at the command prompt. Then type "dir" to view the contents of this directory. There should be at least two files: dashdice.dwg and dice7seg.dwg for this tutorial to work properly.

If you are currently located in the menu of DASH move to the far right of the menu and select Load. The drawing screen will then appear with a command line at the bottom containing the word load. Type in "dashdice" and press return. The drawing will be loaded into the full screen for you to view. Move the cursor around the screen observing the dashed box that follows the cursor. This is the zoom window. Move the cursor so the box is encompassing the area in which you wish to zoom in upon. To zoom in and out so details of the drawing can be better seen, use the "PgUp" and "PgDn" keys from the keyboard. Features that you might wish to look at are the input/output buffers and pins, and how they are represented in symbolic terms within the drawing. The labels given to drawing are called attributes. The attributes define the labels given to certain macros such as I/O pins, logic gates, and the signals running between the two. As the mouse is moved across the attributes, the values are displayed to the right of the viewing area giving greater insight to their definition. These attributes are quite important to the final LCA design through their ability to direct outputs of the design to the actual device pins, and give a macro in a design an actual call to an additional drawing design.

Now zoom out to the full-size drawing picture and place the zoom window around the large rectangular box in the lower right portion of the drawing (not the title box). Zoom in to that area and notice that the box at the top contains the title "dice7seg". This box actually represents a lower level schematic named "dice7seg.dwg" that is implemented as a user-created macro in the top-level design "dashdice.dwg". To view 
this drawing, go back to the menu by pressing the right mouse button. Move to the far right side of the Menu again and select Load. The viewing screen will once again appear with the command line at the bottom. Type "dice7seg" and hit $<$ RETURN $>$. The drawing of the macro dice7seg will be loaded for you to view.

If you wish to bypass the Menu portion of this process, just type "load dice7seg" and it will do the same job as selecting load from the Menu. This section was meant to let a user see the schematic description of the design in which you will soon be downloading to the device. No changes should have been made at this stage of the tutorial, otherwise the final LCA design may not function correctly. You now want to quit out of the schematic capture package one of two ways:

1) Go back in the menu and select "quit".

2) Type "quit" or "q" from where you are currently located.

You should now be back to the DOS prompt, and should be in the directory:

C:>UXACT1_3OTTUTORIAL>

This is the default directory where DASH places all of its design files. It is necessary to be located in this directory for now, since you will be converting the design files to Xilinx netlist files. Please refer to the FutureNet DASH manual Vol. I for an overview of the available operations in the schematic capture tools. Also, refer to Vol. II of the DASH manuals for definitions of each command and the user selected options stored in the application's profiles. 


\section{CONVERTING THE DRAWING FILES}

The first step that a user will make is to prepare the drawings that you have just viewed for post-processing before conversion into a pinlist file. Follow Figure 2 in Chapter II to see the basic design flow. At the DOS prompt, type:

\section{C:XACT1_30TUTORIAL> dcm dashdice.dwg then,}

C:XACT1_30 TUTORIAL > dcm dice7seg.dwg

This will create a total of four files: dashdice.der, dice7seg.der, dashdice.dcm, and dice7seg.dcm. The two der files are a record of the conversion of the drawing files to .dcm files and are not needed for further processing. The two files that we are concerned with are the. $\mathrm{dcm}$ files. DCM is a preprocessor program used in the translation from DASH-LCA schematics to an XNF file. These preprocessor files are the first step in converting the drawing files to pinlist files. The pinlist files contain a list of the connections within and electronic design, consisting of the names of the pins of all symbols in the entire design, and the names of the signals to which each pin connects. To convert the dom files to pin files, type:

\section{C:XACT1_30 TTUTORIAL> pinc dashdice.dcm then,}

\section{C:XACT1_30TTUTORIAL> pinc dice7seg.dcm}

This will create the two files: dashdice.pin and dice7seg.pin. PINC is a pin-list generator program used to translate the preprocessed dcm files to a FutureNet compatible PIN file. For a detailed explanation of the PINC command, refer to Vol. II of the Xilinx XACT Programmable Gate Array manual in Chapter 2.2. The next step is to convert the pin files into Xilinx Netlist Files (XNF) with pin2xnf. The netlist file is a common Xilinx 
format for logic input to the development system, regardless of the source (that is schematic, boolean equations, state machine language). To make the conversion, type:

C:XACT1_30 TTUTORIAL> pin2xnf -P 2064PC68-50 dashdice.pin

The -P option allows the user to select the target Xilinx device that will be used (in this case the XC2064PC). The trailing -50 is the speed grade of the device. The trailing filename is the pinlist file that you just created. As you can observe, you only had to convert the top level design to a netlist file. Since the design dice7seg is included in dashdice, the XNF conversion process searches the top level pinlist file (dashdice.pin) and automatically converts all files contained within it (dice7seg.pin).

\section{CREATING THE LOGIC CELL ARRAY FILE}

Now that you have converted the entire design to the gate-level netlist file, you must convert it to a Configurable Logic Block and Input/Output Block level. This is called a Logic Cell Array (LCA) file. This file will later on be placed and routed into the targeted device. To make this conversion, type:

C:UXACT1_30TTUTORIAL > xnfcvt dashdice.xnf dash.xnf

This command provides the conversion from older XNF files to the most recent version. This is needed since the pin $2 x n f$ conversion is from the older software. Refer to the Xilinx User Guide and Tutorials manual for the best explanation of the xnfcvt command. The XNF filename was changed, so we now have two versions of the same XNF file. At this point the user needs to partition the design logic into CLB and IOB resources with the xnfmap program. To map the dashdice design, type:

C:XACT1_30 TTUTORIAL> xnfmap -p 2064pc68-50 dash.xnf 
When xnfmap has been executed, two files should have been created. The file dash.crf is a cross-reference report showing how xnfmap partitioned the logic. The file dash.map contains the partitioned logic from the XNF file. The dash.map file must then be converted to an LCA file. To convert the partitioned logic into an LCA file, type:

C:XXCT1_30 TTUTORIAL> map2lca -p 2064pc68-50 dash

After the execution of the map2lca program, three files should have been created. The first file, dash.aka, lists the signal prefixes to let the user know which functional block the signal comes from. The file dash.lca is an LCA design file that has yet to be placed or routed. The dash.scp file is a schematic constraints file containing information that assists APR in placement and routing, such as pin and block locations, and net flags from the schematic files. Chapter 5.4 of Vol. II of the Xilinx XACT Programmable Gate Array Development System manual gives a detailed explanation of the map commands.

The final step is to place and route your LCA file into the target device. The Automatic Place and Route procedure (APR), reads an LCA file, generates a new block placement, routes the nets of the design, and writes the result to another LCA file. In APR, there are four steps: 1) Partitioning phase where logic is partitioned into pieces that can be implemented with LCA logic blocks (that is CLB's, IOB's, and so forth); 2) Placement phase where blocks are assigned to a location with the LCA; 3) Routing phase where the signals connecting the blocks are routed using the routing resources of the LCA; 4) Programming phase where the design information is converted into a bit stream which is then loaded into an LCA. The number of possible block placements is the matrix size (\# of CLB's). For the XC2064, this is greater than $10^{89}$ possible placements, not including $\mathrm{I} / \mathrm{O}$ blocks.

A "simulated annealing" algorithm is used to determine the optimal block placement. Annealing refers to the crystallization process in which a metal is melted and 
then slowly cooled back to freezing in order to form highly ordered crystals with few defects. Simulated annealing is an algorithm for finding good solutions to complicated optimization problems in a manner analogous to the physical crystallization of a metal. During each iteration of the simulated annealing placement algorithm, two or more blocks are exchanged at random, and the "routability" of the resulting placement is calculated [60]. This routability is expressed in terms of a "routing score", calculated using a formula that includes the length of the routes, the net weighting, and the number of available routing channels as factors.

The simulated annealing placement algorithm has two phases: the annealing phase and the quenching phase. In the annealing phase, a new placement that results in better routability is always accepted. However, if the new placement is worse, there is still a probability that that it will be accepted, depending on how much worse it is and the current "temperature". The worse the placement, the less likely it will be accepted, although the higher the "temperature", the greater the chance of its acceptance. As the algorithm proceeds, the design is slowly "cooled" by lowering the temperature. As the design cools, blocks move less freely and tend to settle into place. Once the design has sufficiently cooled, the quenching phase is entered. At this stage, only placements of better optimization are accepted.

By default, APR automatically calculates and assigns a starting temperature sufficient to "melt" the design, or in other words, a temperature high enough to allow total scrambling of the initial placement. The algorithm then determines the rate at which the temperature is lowered and when the design has cooled enough to allow quenching. All of this information can be seen as it is happening upon invoking the APR program. To place and route your LCA design, type:

C:IXACT1_30\TUTORIAL > apr dash.lca dashapr.lca 
This step will take several minutes to place and route this design. Larger designs such as the design for the Iterative Logic Unit (ILU) of the Cube Calculus Machine 2.0 (CCM2) may take up to several hours to do the APR step. As you can see, the syntax of the APR statement has the unrouted LCA file, and the placed and routed LCA file which will be distinguished as dashapr.lca. Refer to Chapter 3 of the Xilinx XACT Programmable Gate Array Development System Vol. II manual.

\section{VIEWING THE LOGIC CELL ARRAY FILE}

You now have a complete logic cell array design that is placed and routed. To view your placed and routed design, using the XACT Design Editor by typing at the prompt:

\section{C:UXACTDESIGNS $>$ xde}

This brings you into the XACT development system, allowing you to view, change and download your design to the device. Move the cursor to the Designs menu and select Design. Another menu will pop up and allow you to choose the design which you wish to work with. If you followed the exact syntax that I used for the APR step, the design you want to work with is dashapr.lca. Select this file, and wait.

Once the file is loaded and ready, move the cursor to the Programs Menu and select Editlca. The editing screen will appear, and you will be able to view the layout of the dashdice design. Move the cursor onto the layout area and click down on the left mouse bottom and drag the mouse around. This allows you to scan across the layout while being able to see a global picture of the layout in the lower right corner of the screen. Now, move to where you have a good view of the routing within your local window and let up on the mouse. The global window disappears and you will be able to see the routing from CLB's through switching matrices to other CLB's and I/O Blocks. Move the cursor 
and point to a pin on any CLB or I/O block, and watch the information at the bottom of the screen. It will tell you the current pin being pointed at, what it corresponds to in your design layout, and its net connections. Once you have looked around here, move the cursor to the Misc Menu and select Exit. You will now be out at the Main Menu of $\mathrm{XACT}$ and ready for downloading the dashapr.lca design to the demo board.

\section{DOWNLOADING THE LOGIC CELL ARRAY FILE}

First, install an XC2064 in the demo board's LCA socket and connect the power source to the jumper J3 (pin 2-GND, pin 1-Vcc). Next install the download cable. One end is connected to the parallel port of the computer which has the Xilinx key connected to it. Fit the connector to the key and the other end to the demo board's J1 jumper. Connection to $\mathrm{J} 1$ is obvious due to the keyed pin fitting. When the demo board has been properly prepared, invoke MakeBits from the Programs Menu to access the bitstream and downloading commands. If prompted, select the design file you wish to download (dashapr.lca). Once the MakeBits screen appears, use the Misc Menu's Port command to specify which port the download cable is connected to. For our setup, select LPT1. If the cable is connected properly, a message saying that Port LPT1 is initiated will appear at the bottom of the screen.

Next, invoke the Config Menu's MakeBits command, creating a bitstream file that is present in memory and can be downloaded into an LCA. At this junction, select the Tie option which will internally tie all floating I/O pins low. Then select Done, and the XACT system will create the bitstream file dashapr.bit for downloading. Also created is dashapr.mbo, a record of the MakeBits options that were selected in the process of creating dashapr.bit. Next, invoke the WriteBits command in Config Menu to save the bitstream file to disk. Hit $<\mathrm{CR}>$ for the default filename, which should be dashapr.bit. 
Then, use the Download Menu's Download command to transfer the bitstream into the LCA on the demo board. If prompted "Reset the LCA", press the demo board's Reset switch once. The bottom of the screen should show that the Reset pin has gone low. Then hit $<C R>$ to start the downloading process. Downloading should take 1 to 20 seconds depending upon the speed of the PC. If the download is successful, the message "Done Signal Went High" will appear at the bottom of the screen. If downloading fails, first check the cable connection between the demo board and the PC for proper connection. If download is successful but the demo board doesn't function correctly, check the user switch settings SW1 on the demo board, noting the correct positions of ON/OFF switches.

For this design, a 3-bit Johnson counter is decoded into a seven segment display of 1-6. For proper operation, the seven segment display on the demo board should be rapidly counting from 1-6 until the clock is inhibited by setting SW1 to 0/OFF, effectively stopping the counter at a random number. 